Bundesinstitut
für Bevölkerungsforschung

Belastungen von Kindern, Jugendlichen und Eltern in der Corona-Pandemie

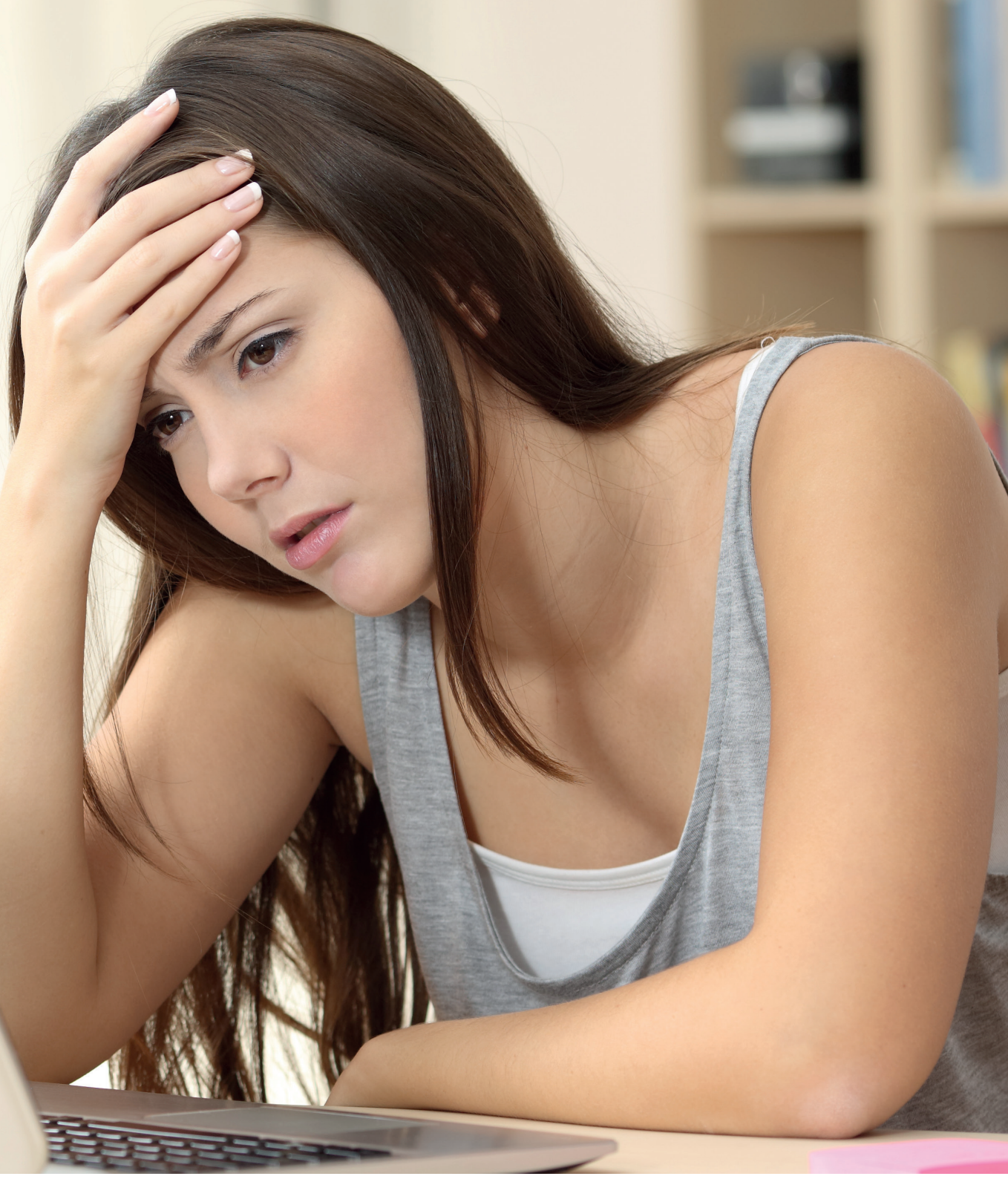




\title{
Belastungen von Kindern, Jugendlichen und Eltern in der Corona-Pandemie
}

\author{
Bundesinstitut für Bevölkerungsforschung \\ Friedrich-Ebert-Allee 4 \\ 65185 Wiesbaden \\ Telefon: 0611-752235 \\ E-Mail: post@bib.bund.de \\ De-Mail:kontakt@bib-bund.de-mail.de \\ Internet: www.bib.bund.de
}

Autorinnen und Autoren:

Martin Bujard ${ }^{a}$, Ellen von den Driesch ${ }^{a}$, Kerstin Ruckdeschel ${ }^{a}$, Inga Laß Carolin Thönnissen ${ }^{b}$, Almut Schumann ${ }^{a}$, Norbert F. Schneider ${ }^{a}$

${ }^{\mathrm{a}} \mathrm{BiB},{ }^{\mathrm{b}}$ Universität Köln

Layout, Satz und Redigierung:

Christian Fiedler, Sybille Steinmetz, Britta Müller

Titelbild:

Adobe Stock | Antonioguillem

Vervielfältigung und Verbreitung, auch auszugsweise, mit Quellenangabe gestattet

C Bundesinstitut für Bevölkerungsforschung (BiB) 2021

urn:nbn:de:bib-var-2021-028

DOI: https://doi.org/10.12765/bro-2021-02

ISSN 2702-7651 (Print)

ISSN 2702-766X (Online) 


\section{Belastungen von Kindern, Jugendlichen und Eltern in der Corona-Pandemie}




\section{Inhalt}

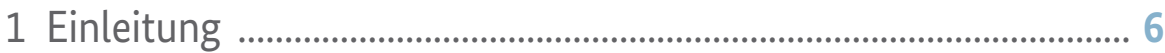

2 Corona-Inzidenzen und Lockdown-Regelungen ........................... 8

3 Anzahl von Kindern, Jugendlichen und Familien ......................... 12

4 Bildung - Weniger Lernzeit und Lernerfolge zuhause ................ 16

5 Belastungen von Kindern und Jugendlichen im Lockdown ...... 24

6 Belastungen von Eltern im Lockdown ........................................... 46

7 Warum soziale Ungleichheiten die Belastungen verstärken ..... 67

8 Fazit und Empfehlungen ................................................................ 72

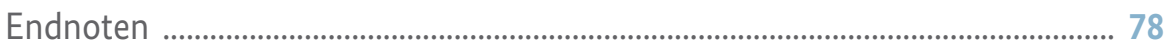

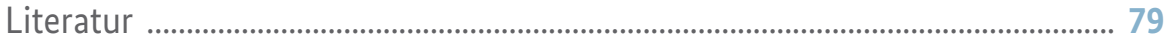




\section{Einleitung}

Die Corona-Pandemie hat das Leben in Deutschland seit nunmehr 17 Monaten (Stand Juli 2021) grundlegend verändert, und nachweislich wurden bereits über 3,7 Mio. Menschen in Deutschland mit dem Virus infiziert. Bund und Länder haben einschneidende Infektionsschutzmaßnahmen erlassen, um die Ausbreitung zu bremsen und eine Überforderung des Gesundheitssystems zu vermeiden, v.a. Hygienemaßnahmen und Kontaktbeschränkungen. Diese Maßnahmen haben erhebliche Einschränkungen und Beeinträchtigungen der Bildung, der Erwerbsarbeit, des alltäglichen Lebens und auch der psychischen Gesundheit zur Folge. Es ist zu begrüßen, dass die Auswirkungen der Maßnahmen auf die Familien sowie die Bildung und Gesundheit von Kindern angesichts der Größenordnung der von Kita- und Schulschließungen betroffenen Gruppe (11 Mio. Kinder und Jugendliche sowie deren Eltern) in den Fokus von Öffentlichkeit und Politik rückt.

Die gesundheitlichen Auswirkungen der Corona-Krise und der damit verbundenen Schulschließungen lassen sich nicht anhand eines Indikators messen, da sie vielfältig sind und bspw. bei psychischen Belastungen schwer zu diagnostizieren und messen sind. So kennt man zwar tagesgenau die Corona-Inzidenzen, jedoch kaum konkrete Zahlen zu den Größenordnungen der anderweitigen gesundheitlichen Beeinträchtigungen. Allerdings gibt es zunehmend Hinweise darauf, dass viele Kinder, Jugendliche und auch Eltern nicht nur gestresst und in ihrer Lebensqualität eingeschränkt sind, sondern auch von ernsthaften psychischen Folgen beeinträchtigt sind.

Gegenwärtig ist die Forschungslage noch begrenzt, da nur wenige Datenerhebungen aus der Zeit des zweiten Lockdowns vorliegen. Auch unterscheiden sich vorhandene Studien in erheblichem Maße danach, wann, also in welcher Phase der Pandemie, die Befragung stattfand. So macht es einen großen Unterschied, ob Personen zu Beginn der Pandemie und des ersten Lockdowns im März/April 2020, später im ersten Lockdown im Mai/Juni 2020, als sich vieles bereits eingependelt hatte, in den lockdownfreien 
Sommermonaten, im Teillockdown November 2020 oder während des zweiten Lockdowns Anfang 2021 befragt wurden.

Es gibt eine Vielzahl von Studien zu den Effekten der Corona-Krise auf Familien, jedoch mit unterschiedlicher Qualität: Die Stichproben sind häufig nicht repräsentativ, da es sich um Convenience- oder Schneeballstichproben handelt, bei denen die Befragten nicht zufällig ausgewählt werden und somit häufig eine stark verzerrte und nicht der Grundgesamtheit entsprechende Stichprobe darstellen. Auch basieren viele Studien auf Querschnittsdaten, die keinen Vergleich zu der Zeit vor der Pandemie ermöglichen. Derartige Studien werden hier teilweise ergänzend erwähnt, Schwerpunkt dieser Publikation sind jedoch Analysen mit qualitativ hochwertigen Daten, die repräsentativ für die Bevölkerung in Deutschland sind und einen Vergleich im Zeitverlauf ermöglichen mithilfe von Längsschnittdaten.

Diese Studie gibt einen Überblick über Auswirkungen der Corona-Pandemie und den damit verbundenen Maßnahmen auf Familien in Deutschland auf Basis des gegenwärtigen Wissensstands. Grundlage sind erstens eigene Analysen zur psychischen Belastung mit dem deutschen Familienpanel pairfam. Pairfam ermöglicht durch jährliche Wiederholungsbefragungen einen Vergleich der Situation vor der Pandemie mit der Zeit seit Ausbruch der Pandemie und basiert auf repräsentativen Daten. Zweitens werden eigene Analysen auf Basis des Mikrozensus, der amtlichen Statistik und des Sozio-oekonomischen Panels herangezogen. Drittens erfolgt ein breiter Überblick über aktuelle Studien zur Situation von Kindern, Jugendlichen und Eltern hinsichtlich ihrer gesundheitlichen Belastung, psychosozialen Situation sowie zur Bildungssituation der Kinder und Jugendlichen. Insgesamt soll die hier vorliegende Studie eine belastbare Informationsbasis für Entscheidungsträgerinnen und Entscheidungsträger darstellen und verdeutlichen, an welchen Stellen angesetzt werden sollte, um Eltern, Kinder und Jugendliche bei der Bewältigung der Folgen der Pandemie adäquat zu unterstützen. 

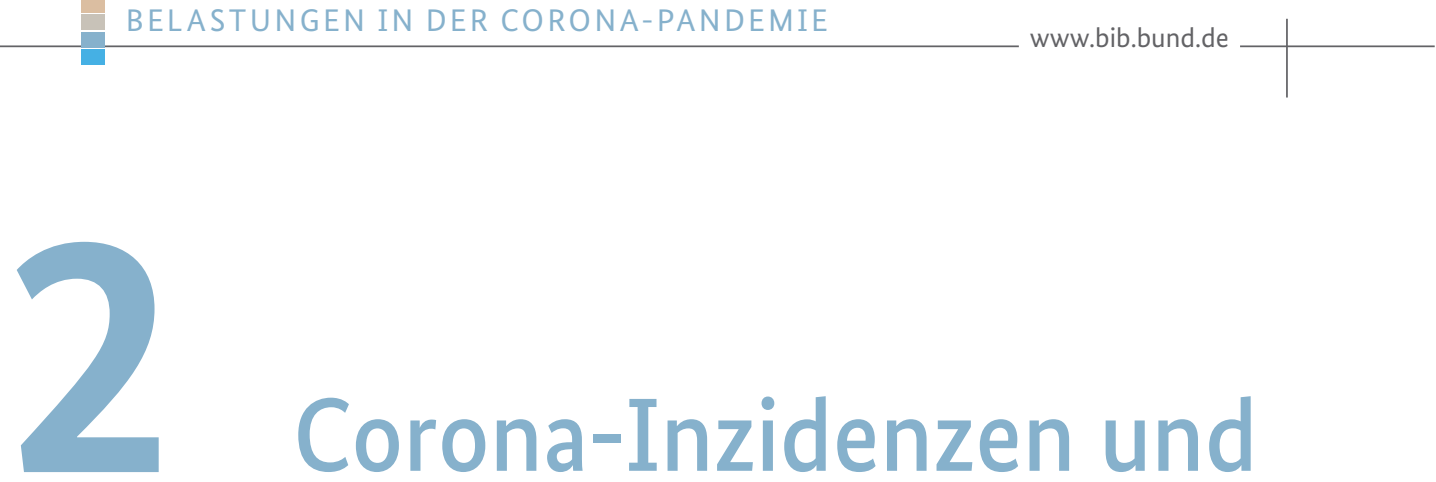

\section{Corona-Inzidenzen und Lockdown-Regelungen}

Das Infektionsgeschehen mit bislang drei Wellen und die damit verbundenen politischen Maßnahmen waren sehr dynamisch und wechselhaft (Abb. 1). Im ersten Lockdown wurden die Kita- und Schulschließungen relativ spät beendet. Beim zweiten Lockdown, Anfang November 2020, wurden Kitas und Schulen zunächst bis Mitte Dezember offengelassen. Ab Mitte Dezember wurden auch Kitas und Schulen geschlossen. Dieser zweite Lockdown wurde mehrfach verlängert, wobei die Bundesländer seit März 2021 in unterschiedlichem Tempo die Schulen - vor allem die Grundschulen, oft im Wechselunterricht - wieder öffnen konnten. Vor Ostern gab es bereits mehrere Lockerungen, in mehreren Bundesländern wurde bei Schulen der Sekundarstufe der Präsenzunterricht allerdings erst im Mai wieder - zunächst als Wechselunterricht - zugelassen.

Nach einem deutlichen Anstieg der Infektionen galt zwischen dem 24. April und Ende Juni 2021 die bundeseinheitliche Notbremse im Infektionsschutzgesetz, die angesichts der vorherigen Vielzahl unterschiedlicher regionaler Regelungen einheitliche Grenzen setzte. Demnach galten bei einer Sieben-Tage-Inzidenz von über 100 in einem Landkreis oder einer kreisfreien Stadt strikte Kontaktbeschränkungen, eine nächtliche Ausgangssperre, geschlossener Einzelhandel und weiterhin geschlossene Freizeiteinrichtungen und Gastronomie. Bei Inzidenzen bis 150 war das Einkaufen mit Test und Termin ("Click \& Meet") möglich. In der Arbeitswelt galt eine Pflicht zum Homeoffice, wo dies möglich war; eine konsequente Test- und Maskenpflicht wie bei den Schulen war nicht vorgeschrieben, wurde aber von vielen Unternehmen freiwillig umgesetzt. Für Schulen war ab einer Inzidenz von 100 Wechselunterricht und Testpflicht vorgeschrieben, ab einer Inzidenz von 165 mussten die Schulen geschlossen werden und in den Distanzunterricht wechseln. Die Bundesländer hatten die Möglichkeit, strengere Regeln und Ergänzungen einzuführen. Die Bundesnotbremse definierte somit die Mindestanforderung, welche Regelungen in Abhängigkeit von der Inzidenz in Kraft treten mussten. 
Abbildung 1 gibt auch einen Überblick über die Dauer der Schulschließungen. Die weitgehend vollständige Schulschließung war im ersten Lockdown vom 23.03.2020 bis 05.05.2020, insgesamt 44 Tage. Anschließend folgte eine partielle Öffnung, in der in mehreren Bundesländern die Sekundarstufe länger geschlossen war und es teilweise bis zu den Sommerferien Wechselunterricht gab. Rechnet man bis zum 03.07.2020, den durchschnittlich letzten Schultag vor den Sommerferien, sind es 59 Tage mit partieller Schulschließung, und somit insgesamt 103 Tage ohne regulären Präsenzunterricht im ersten Lockdown. Die Zeit ganz ohne Präsenzunterricht betrug für die meisten Kinder und Jugendlichen zwischen eineinhalb und drei Monaten.

Im zweiten Lockdown reichte die weitgehend vollständige Schulschließung vom 16.12.2020 kurz vor Weihnachten bis zum 14.02.2021, insgesamt 61 Tage. Anschließend folgte die partielle Öffnung, insbesondere für Grundschulkinder. Bei den Sekundarstufen gab es erhebliche Unterschiede nach Bundesländern, bspw. hat Rheinland-Pfalz den Wechselunterricht ab dem 18.03.2021 eingeführt, in Hessen wurden die Jahrgangsstufen sieben und höher bis zur Einführung der Bundesnotbremse im Distanzunterricht beschult und je nach Inzidenz ab dem 06.05.2021 im Präsenzunterricht, zunächst im Wechselmodell. Seit Anfang Juni 2021 sind die Inzidenzen in den meisten Kreisen so gering, dass die Bundesnotbremse vollen Präsenzunterricht zulässt. Am 7. Juni 2021 lag die Inzidenz im Bundesschnitt bei 24. Nimmt man dies als Endpunkt, hat die partielle Schulschließung im zweiten Lockdown 112 Tage gedauert; insgesamt summiert sich die vollständige und partielle Schulschließung des zweiten Lockdowns auf 173 Tage.

Die Zeit ohne Präsenzunterricht betrug im zweiten Lockdown mindestens zwei Monate für die Grundschülerinnen und -schüler. In einigen Bundesländern waren Schülerinnen und Schüler der siebten bis zwölften Klasse von Mitte Dezember 2020 bis Mitte Mai 2021 nicht in der Schule. Mehrere Millionen Kinder und Jugendliche haben in diesem zweiten Lockdown vier oder fünf Monate keine Schule in Präsenz besucht und diese Monate stattdessen vor dem Computer im Kinderzimmer verbracht und an digitalem Distanzunterricht teilgenommen oder konventionell Aufgaben mit Stift und Papier bearbeitet. Der zweite Lockdown war für fast alle Schülergruppen in Deutschland länger als der erste. 


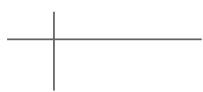

35.000

30.000

25.000
10.03.2020

Empfehlung des Krisenstabs des Bundes, Veranstaltungen mit mehr als 1.000 Personen abzusagen

Einreisestopp für Menschen aus Drittstaaten und Einschränkungen von Reisen in der EU

Zahlreiche Geschäfte, Gastronomie, Kulturhäuser, Sportvereine, Kirchen müssen schließen Schul
ür M
d Eins
chäf

Bundesweite Kontaktbeschränkungen:

, Geschäfte etc.

06.05.2020

Schrittweise Rückkehr der Beschulung im Präsenzunterricht

Schrittweise Öffnung aller Geschäfte, der Gastronomie, Kultur- und Sporteinrichtungen unter Auflagen b

30.04.2020

Öffnungen Spielplätze, Museen etc. unter Auflagen

15.04.2020

Maskenpflicht im ÖPNV und beim Einkauf

30.01.2020

WHO ruft internationalen Gesundheitsnotstand aus

Teilweise Lockerung von Kontaktbeschränkungen
27.08.2020

Beschluss keiner weiteren Öffnung, Verbot von Großveranstaltungen und Aufruf, Kontakte auf Minimum zu beschränken sowie Verlängerung des Kurzarbeitergeldes

\subsubsection{0}

Testpflicht für Reiserückkehrende aus Risikogebieten

16.07.2020

14-tägige Quarantäne für Einreisende/ Rückkehrende aus Risikogebieten
5.000

27.01.2020

Erster bestätigter Covid-

19-Fall in Deutschland
0

Jan.

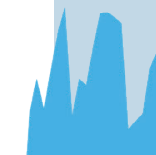

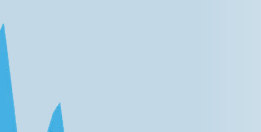

17.06.2020

Beschlüsse umfangreicher politischer Maßnahmen zur Bekämpfung der negativen Pandemiefolgen: Senkung der Umsatzsteuer, Kinderbonus etc.
14.09.2020

Weitgehender Präsenzunterricht in allen Schulen und Bundesländern

\section{Abbildung 1: Zeitliche Übersicht der zentralen Maßnahmen zur Eindämmung der Corona-Pandemie in Deutschland}




\section{Anzahl von Kindern, Jugendlichen und Familien²}

Am Anfang der Pandemie, zu Beginn des Jahres 2020, lebten in Deutschland 13,7 Mio. Kinder und Jugendliche unter 18 Jahren - das entspricht etwa einem Sechstel der Bevölkerung. Der Anteil der über 60-Jährigen ist mit 23,7 Mio. deutlich größer. In allen Altersgruppen der Kinder gibt es etwa 5 bis $6 \%$ mehr Jungen als Mädchen (Abb. 2). 2,37 Mio. Kinder sind im Alter unter 3 Jahren, ähnlich viele sind im Kindergartenalter von 3-5 Jahren. Im Schulalter sind die Jahrgänge etwas kleiner. Wichtig für die folgenden Analysen sind die Größenordnungen der Schulkinder im Alter von unter 12 Jahren, da für diese eine Notbetreuung vorgesehen war. Im Alter von 6-11 Jahren sind es 4,4 Mio. Kinder und im Alter von 12-17 Jahren 4,5 Mio. Jugendliche.

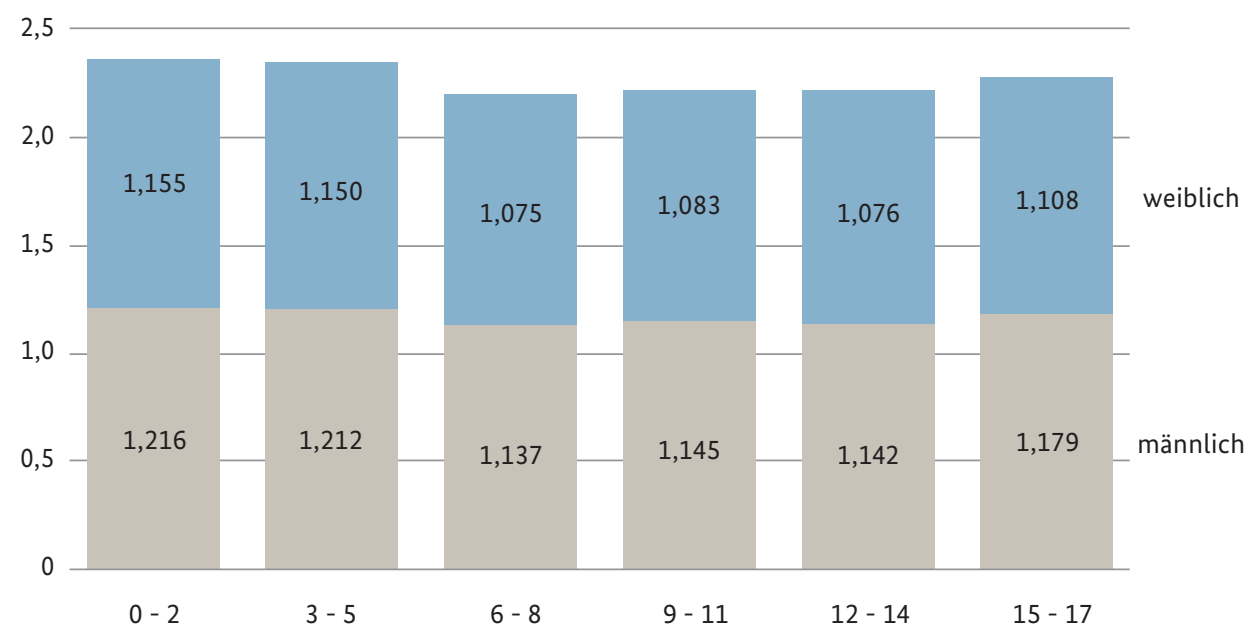

Abbildung 2: Zahl der Kinder nach Alter in Mio. (Stand 01.01.2020)

Quelle: Statistisches Bundesamt 2021, eigene Berechnung. 0-2 Jahre umfasst alle Kinder der Jahrgänge 2017-2019 etc. 
Nicht alle der 13,7 Mio. Kinder und Jugendliche besuchen Kitas oder Schulen. Die Schließungen von Kinderbetreuungseinrichtungen und Schulen betrafen unmittelbar 11,1 Mio. Kinder und Jugendliche in Deutschland sowie ihre Familien. Während die meisten Kinder ab drei Jahren in Kitas oder Kindergärten gehen, und alle Kinder im schulpflichtigen Alter die Schule besuchen, fällt der Anteil der unter Dreijährigen, die eine Betreuungseinrichtung besuchen, deutlich geringer aus: Bei unter Einjährigen ist die Betreuungsquote sehr gering bei knapp $2 \%$. Bei einjährigen Kindern liegt sie bei $37 \%$, bei den Zweijährigen bei $63 \%$.

Im Schuljahr 2018/19 wurden 8,2 Mio. Schülerinnen und Schüler an allgemeinbildenden Schulen in Deutschland unterrichtet. Nach vorläufigen Daten sind es im Schuljahr 2019/20 rund 8,3 Mio. Dazu kommen 2,4 Mio. Schülerinnen und Schüler an beruflichen Schulen.

\begin{tabular}{lccccrr}
\hline & $\begin{array}{c}\text { betreute } \\
\text { Kinder } \\
\text { 0-2 Jahre }\end{array}$ & Kitas & Primärstufe & $\begin{array}{c}\text { Sekundar- } \\
\text { stufe I } \\
\text { (Klassen 5-10) }\end{array}$ & $\begin{array}{c}\text { Sekundar- } \\
\text { stufe II } \\
\text { (Klassen 11-13) }\end{array}$ & Gesamt \\
\hline Baden-Württemberg & 96.465 & 291.235 & 403.227 & 586.433 & 102.339 & 1.479 .699 \\
\hline Bayern & 109.549 & 333.021 & 462.842 & 660.585 & 114.123 & 1.680 .120 \\
\hline Berlin & 51.951 & 99.449 & 132.729 & 172.511 & 48.647 & 505.287 \\
\hline Brandenburg & 36.529 & 62.576 & 90.428 & 118.556 & 30.139 & 338.228 \\
\hline Bremen & 5.851 & 16.374 & 22.883 & 32.967 & 8.892 & 86.967 \\
\hline Hamburg & 28.699 & 48.945 & 65.864 & 88.643 & 30.321 & 262.472 \\
\hline Hessen & 57.749 & 160.694 & 226.207 & 322.145 & 62.121 & 828.916 \\
\hline Mecklenburg-Vorpom. & 22.825 & 39.101 & 57.500 & 73.425 & 16.569 & 209.420 \\
\hline Niedersachsen & 72.011 & 197.666 & 287.667 & 457.921 & 70.569 & 1.085 .834 \\
\hline Nordrhein-Westfalen & 147.171 & 449.648 & 664.250 & 962.550 & 252.767 & 2.476 .386 \\
\hline Rheinland-Pfalz & 35.933 & 104.690 & 143.190 & 209.781 & 50.107 & 543.701 \\
\hline Saarland & 7.415 & 21.937 & 31.764 & 44.849 & 11.402 & 117.367 \\
\hline Sachsen & 58.242 & 106.509 & 149.132 & 185.885 & 33.852 & 533.620 \\
\hline Sachsen-Anhalt & 31.488 & 51.542 & 76.449 & 96.645 & 18.807 & 274.931 \\
\hline Schleswig-Holstein & 26.860 & 68.602 & 106.197 & 146.510 & 35.880 & 384.049 \\
\hline Thüringen & 29.745 & 54.123 & 75.507 & 96.733 & 19.671 & 275.779 \\
\hline Deutschland gesamt & 818.483 & 2.106 .112 & 2.995 .836 & 4.256 .139 & 906.206 & 11.082 .776 \\
\hline & & & & & & \\
\hline
\end{tabular}

Tabelle 1: Zahl der Kinder in Kitas und Tagespflege sowie der Schülerinnen und Schüler an allgemeinbildenden Schulen nach Bundesländern (2019)

Datenquelle: Bujard et al. 2020, S. 12. 
Diese Werte unterscheiden sich erheblich nach Bundesländern, bedingt durch deren Einwohnerzahl; so sind es in Nordrhein-Westfalen 1,88 Mio. Schülerinnen und Schüler, 450.000 Kinder im Kindergarten und 147.000 unter Dreijährige in öffentlicher Kinderbetreuung. Auch in Baden-Württemberg, Bayern und Niedersachsen liegt die Gesamtzahl der Kinder und Jugendlichen in Betreuungseinrichtungen und Schulen über 1 Mio. Tabelle 1 gibt einen Überblick zu den jeweiligen Zahlen in verschiedenen Abschnitten des Bildungssystems für sämtliche Bundesländer.

Nachdem soeben die Anzahl der Kinder und Heranwachsenden in Betreuungs- bzw. Bildungseinrichtungen aufgezeigt wurde, folgt nun ein Blick auf die Eltern, die mehrheitlich ebenso von den Schließungen der Betreuungseinrichtungen betroffen sind. Laut aktuellem Mikrozensus gab es $2018^{1}$ insgesamt 14,6 Mio. Eltern, die mit minderjährigen Kindern zusammenlebten und über 10,5 Mio. Eltern mit einem jüngsten Kind unter 12 Jahren (Abb. 3). Betrachtet man ausschließlich Eltern von Kindern unter 12 Jahren, sind es 4,8 Mio. Väter und 4,8 Mio. Mütter, die als (Ehe-)Paar mit den Kindern zusammenleben sowie 78.000 alleinerziehende Väter und 827.000 alleinerziehende

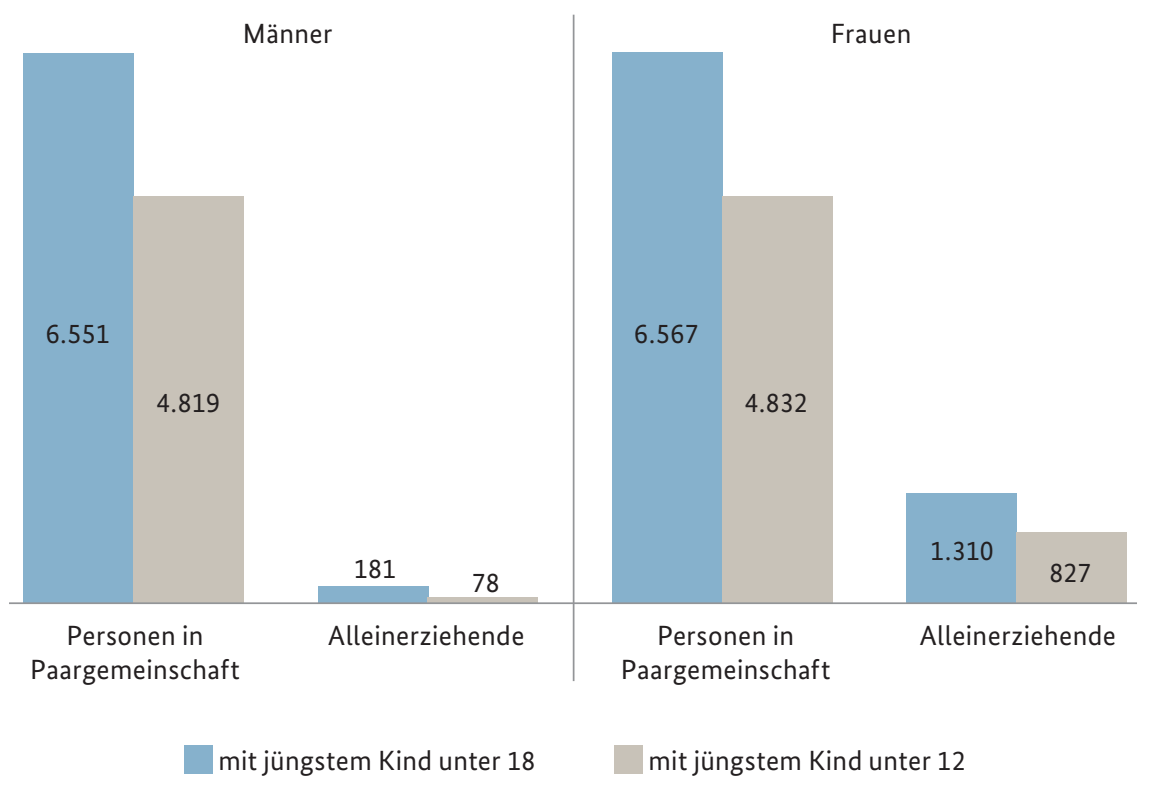

Abbildung 3: Zahl der Eltern mit minderjährigen Kindern in Tsd. (Stand 2018)

Quelle: Bujard et al. 2020b, S. 14. 
Mütter. Diese rund 10,5 Mio. Eltern von unter 12-jährigen Kindern sind in besonderem Maße von den Kita- und Schulschließungen betroffen, da für diese Altersgruppe eine Betreuung der Kinder zuhause durch die Eltern erwartet wird. Diese Altersgrenze ist beispielsweise maßgeblich für die Entgeltfortzahlung.

Die beschriebenen Zahlen stehen für Personengruppen, die durch die Corona-Pandemie zahlreiche, gravierende Einschnitte in die normalen Lebens-, Arbeits- und Bildungsabläufe erfahren haben, wie es sie in Deutschland seit dem Zweiten Weltkrieg nicht mehr gegeben hat. Während der lockdownbedingten Schulschließungen mussten Eltern eventuell ergänzend zu digitalen Unterrichtsformen - häufig die Rolle der Lehrperson übernehmen, indem sie für eine adäquate Lernumgebung und einen strukturierten Tagesablauf sorgten sowie bei Schulaufgaben unterstützen mussten. Dies sollten die Eltern zusätzlich zu ihren beruflichen Tätigkeiten leisten. 

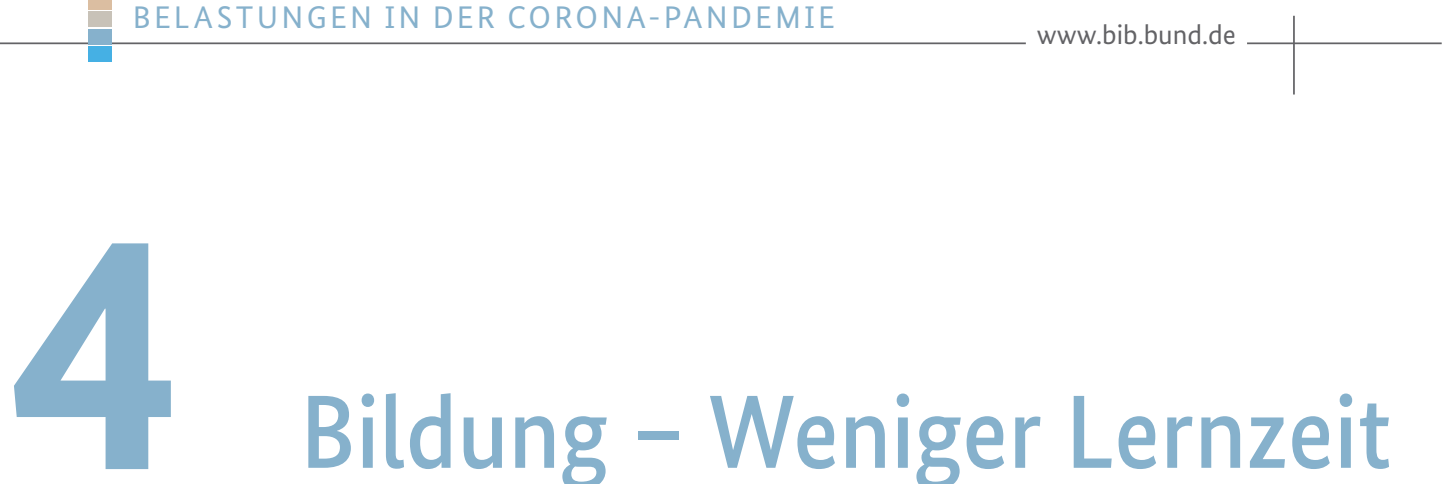

\section{Bildung - Weniger Lernzeit und Lernerfolge zuhause}

\subsection{Lernzeiten}

Bereits Kinder im Vorschulalter sind von den Schließungen der Betreuungseinrichtungen in ihrem Lernen betroffen. Kinder in diesem Alter lernen durch soziale Interaktion im familiären Kreis und im sozialen Umfeld. Besonders deutlich wird dies beim Spracherwerb, der in einem Zeitfenster ungefähr vom dritten bis zum siebten Lebensjahr stattfindet. Erfahrungen, die in diesem Lebensalter nicht gemacht wurden, lassen sich nur sehr schwer oder überhaupt nicht mehr kompensieren. Gerade für Kinder mit Migrations- oder Fluchtgeschichte, aber nicht nur für diese, ist der Spracherwerb zentral (Leopoldina 2021) und Kinderbetreuung ein entscheidender Faktor (Bujard et al. 2020a). Zwar wurden für diese Gruppe von Kindern keine Studien während des ersten oder zweiten Lockdowns durchgeführt, aber es sind Defizite in der sprachlichen und sozialen Entwicklung aufgrund der Schließungen zu erwarten, so dass der von der Bundesregierung geforderte Ausbau der Sprachförderung in Kindertagesstätten gerechtfertigt erscheint, ebenso die Entwicklung einer standardisierten Frühdiagnostik (BMBF und BMFSFJ 2021).

Für Schulkinder und jugendliche Schülerinnen und Schüler bedeuten Schulschließungen, dass zuhause gelernt und gearbeitet wird. Damit entfällt der Schulweg, die Schule als sozialer Raum, das Schulgebäude als Ort, der mit Lernen assoziiert wird und ein extern vorgegebener standardisierter Tagesrhythmus, der, je nach Schultageslänge, bis in den Nachmittag Arbeits- und Erholungszeiten vorgibt. Dies wirkt sich auch auf die Lernzeiten der Schülerinnen und Schüler aus. Mehrere Studien - Oxford-Studie, Nationales Bildungspanel, ifo-Bildungsbarometer, Schulbarometers IBB oder JIMplus Corona-Studie - zeigen, dass das Homeschooling im Lockdown zu deutlich geringeren Lernzeiten bzw. wahrscheinlich auch Lernerfolgen führt. Im politischen und öffentlichen Diskurs wird seit längerem u.a. zuletzt durch die Ständige Wissenschaftliche Kommission der Kultusministerkonferenz (KMK) (StäwiKo 2021), das Bundesjugend- 
kuratorium (BJK 2021) und die Nationale Akademie der Wissenschaften - Leopoldina (Leopoldina 2021) gefordert, pandemiebedingte Lernrückstände aufzuholen und Unterstützungsmaßnahmen, auch im außerschulischen Bereich, (weiter) zu entwickeln.

Das Nationale Bildungspanel (NEPS) hat zwischen Mai und Juni 2020 eine Zusatzbefragung durchgeführt, an der insgesamt 1.452 Eltern von Kindern, die während des ersten Lockdowns die achte Klasse besuchten, teilnahmen (NEPS-C). Augenfällig war ein deutlicher Rückgang der wöchentlichen Lernzeit. Während unter normalen Bedingungen eine Schulwoche ca. 30 Stunden umfasst, mit zusätzlichen Zeiten für Lernen und Hausaufgaben, haben die Schülerinnen und Schüler während des ersten Lockdowns im Mittel 16 Stunden in die Schule investiert. Bei einem Fünftel lag die Lernzeit unter acht Stunden, bei einem Fünftel über 20 Stunden. Dabei fanden sich keine signifikanten Unterschiede nach Geschlecht, Schulform oder Bildung der Eltern. Anders stellt sich die Situation dar, wenn es um die Selbsteinschätzung von Eltern geht, inwieweit sie ihre Kinder inhaltlich beim Lernen zuhause unterstützen konnten. Insgesamt 14 \% der Eltern mit akademischem Bildungshintergrund im Vergleich zu 31 \% der Eltern ohne waren der Meinung, dass ihre Fähigkeiten eher nicht oder überhaupt nicht ausreichten (Wolter et al. 2020).

Zu ähnlichen Ergebnissen bezüglich der Lernzeiten kommt auch das ifo-Bildungsbarometer, das Informationen für beide Lockdowns im Vergleich zur Vor-Corona-Zeit erhoben hat (Wößmann et al. 2020a, 2021). Dazu haben nach dem ersten Lockdown im Juni 2020 insgesamt 1.099 Eltern Angaben zu ihrem jüngsten Schulkind gemacht und im März und April 2021 nochmals 2.122 Eltern, so dass Kinder aller Altersgruppen und Schularten repräsentiert sind ${ }^{3}$. Im Durchschnitt verbrachten die Kinder im ersten Lockdown 3,6 Stunden pro Werktag mit Lernen für die Schule (inkl. Schulbesuch in Notbetreuung), während es vor den Schulschließungen 7,4 Stunden waren (Schulbesuch 5,9 Stunden + Lernen für die Schule 1,5 Stunden), also mit $49 \%$ nur knapp die Hälfte der Zeit. Im zweiten Lockdown ist die Lern- und Arbeitszeit der Kinder im Vergleich zum ersten Lockdown um 0,7 Stunden auf 4,3 Stunden pro Werktag angestiegen, zum Großteil um tatsächliche Lernzeit (0,6 Stunden) und nur zu einem geringen Anteil (0,1 Stunden) durch Schulbesuch, z. B. in Notbetreuung, so dass ein Niveau von ca. 58 \% der Zeit vor der Pandemie erreicht wurde (Abb. 4). Dabei wurden im ersten Lockdown keine wesentlichen Unterschiede nach Bildung der Eltern gefunden, stattdessen nach Leistungsstärke der Kinder (gemessen am Notendurchschnitt in den Fächern Deutsch und Mathe vor dem Lockdown): Leistungsstärkere Kinder verbrachten rund eine halbe Stunde mehr mit Lernen. Im zweiten Lockdown waren diese Unterschiede nicht mehr vorhanden, was die Autorinnen und Autoren der 
Studie als Erfolg durch bessere Distanzunterrichtskonzepte deuten (Wößmann et al. 2021, S. 47). Die verbleibende tägliche Zeit füllten die Kinder mit etwas mehr Lesen, kreativen Tätigkeiten und Bewegung. Hier fand ein Anstieg von durchschnittlich 2,9 auf 3,2 Stunden im ersten Lockdown statt, was aber im zweiten Lockdown wieder auf 2,9 Stunden zurückging. Die Zeit für Fernsehen, Computer und Handy stieg wesentlich deutlicher um durchschnittlich 1,2 Stunden von 4,0 auf 5,2 Stunden im ersten Lockdown und sank im zweiten wieder etwas auf 4,6 Stunden. Sie lag damit nicht nur immer noch deutlich über dem Niveau vor der Corona-Pandemie, sondern war auch höher als die täglich mit schulischen Aktivitäten verbrachte Zeit. Im ersten Lockdown zeigte sich, dass die Unterschiede nach Leistungsstärke besonders ausgeprägt waren, d. h. leistungsschwächere Kinder starteten bei der Mediennutzungszeit von einem höheren Niveau vor der Pandemie und verzeichneten zusätzlich einen stärkeren Anstieg (durchschnittlicher Zuwachs: 1,7 Stunden zu 1,0 bei Leistungsstärkeren) (Wößmann et al. 2020a, 2021).

Die Zunahme der Lernzeit lässt auf Fortschritte in der Digitalisierung und der Organisation des Distanzunterrichts schließen. Während Lehrerinnen und Lehrer im ersten Lockdown vor einer völlig unerwarteten, neuen Situation standen und unter technisch beschränkten Möglichkeiten improvisieren mussten, hatten sie im zweiten Lockdown bereits Erfahrungen gesammelt und Zeit, sich auf didaktisch adäquaten Distanzunterricht einzustellen. So hatten z. B. im zweiten Lockdown $26 \%$ der Schülerinnen und Schüler täglich gemeinsamen Online-Unterricht im Vergleich zu $6 \%$ im ersten Lockdown, und während im ersten Lockdown $45 \%$ nie gemeinsamen digitalen Unterricht hatten, waren dies im zweiten nur noch 18 \% (Wößmann et al. 2021). Entsprechend fällt auch die Beurteilung der Eltern aus, von denen im Frühjahr 202064 \% der Meinung waren, ihr Kind würde zuhause „viel weniger“ lernen als in der Schule, im Winter 2020/21 dagegen mit 54 \% etwas weniger Eltern diese Ansicht vertraten (Wößmann et al. 2021, S. 44). Wahrscheinlich ist aber, dass bei einer Lernzeit im zweiten Lockdown, die nur 58 \% des Niveaus vor der Corona-Pandemie betrug, Lernrückstände zu erwarten sind, selbst dann, wenn der Distanzunterricht didaktisch adäquat durchgeführt wurde. Der zurückgegangene Anteil an Eltern, die die Aussage verneinen, dass ihre Kinder „viel weniger“ gelernt hätten, bedeutet nicht, dass sie mit dem Unterricht zuhause zufrieden waren. Betrachtet man die Meinung der Eltern im zweiten Lockdown differenzierter, so war mehr als die Hälfte (56 \%) der Meinung, ihre Kinder würden zuhause pro Stunde eher weniger lernen als in der Schule und nur $22 \%$ der Eltern waren der gegenteiligen Auffassung. Dabei war weniger die technische Ausstattung als die Fähigkeiten der Kinder zur Selbstmotivation, Selbstorganisation und Konzentration das Problem (Wößmann et al. 2021). 

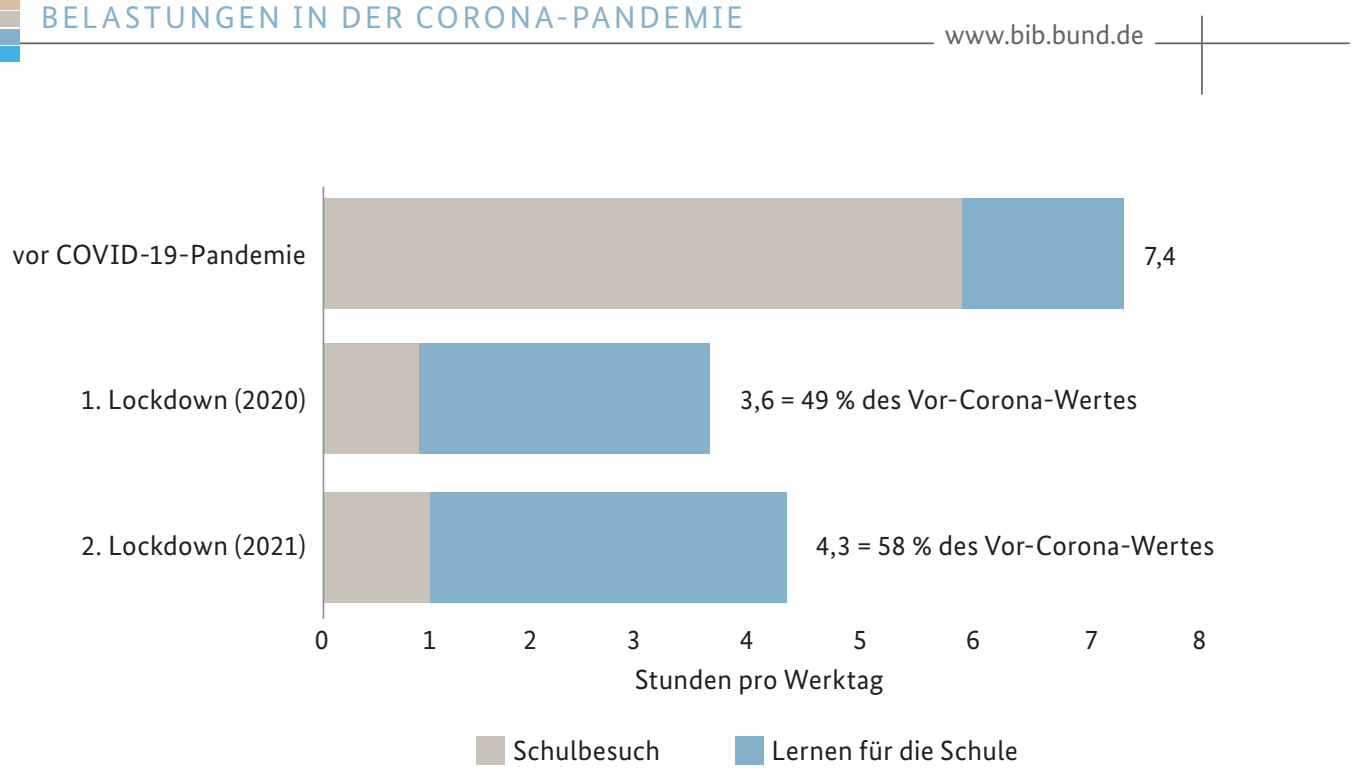

Abbildung 4: Zeit für schulische Aktivitäten vor der Corona-Pandemie und während des ersten und des zweiten Lockdowns

Quelle: Wößmann et al. 2020b, 2021. Die Werte für Schulbesuch während der Lockdowns beziehen sich auf Notbetreuung, anteilig berechnet.

Die Ergebnisse des Schulbarometers des Instituts für Bildungsmanagement und Bildungsökonomie, das vom 24.03. bis zum 05.04.2020 in Deutschland, Österreich und der Schweiz durchgeführt wurde, bestätigen diese Befunde. Sie zeigen auch, dass das Lernen zuhause von den Schülerinnen und Schülern durchaus unterschiedlich beurteilt wird. Die Mehrheit der Schülerinnen und Schüler glaubte nicht, dass sie zuhause mehr gelernt hätten als im Unterricht (51 \%), im Gegensatz zu einem Viertel, das der gegenteiligen Meinung war. In qualitativen Zusatzinterviews wurden als Gründe für eine gute Bewertung des Unterrichts zuhause die Berücksichtigung des individuellen Lerntempos und die ungestörtere Lernatmosphäre angeführt (Huber und Helm 2020; Huber et al. 2020). Die Chancen des Homeschoolings, wie z. B. selbstreguliertes Lernen, hängen allerdings stark von der Leistungsstärke der Schülerinnen und Schüler vor der Schulschließung ab. Vor allem die Lesekompetenz und die Anstrengungsbereitschaft waren ausschlaggebend für eine hohe Motivation und damit einen guten Lernerfolg beim Arbeiten zuhause. Das bedeutet im Gegenschluss, dass die Schulschließungen die Leistungsunterschiede zwischen den Schülerinnen und Schülern weiter vergrößern (Lockl et al. 2021; Huber et al. 2020). Zu ähnlichen Ergebnissen hinsichtlich der Bedeutung der Leistungsbereitschaft kommen auch Dietrich et al. (2021) auf Basis der Berufsorientierungsstudie BerO, wobei sie allerdings Unterschiede nach sozialem Status der Eltern finden. Sie zeigen unter anderem, dass die soziale Her- 
kunft die Leistungsbereitschaft im Homeschooling beeinflusst und dadurch soziale Ungleichheit bei Schülerinnen und Schülern verstärkt. Ähnlich wirken eine schlecht ausgestattete Lernumgebung, wenig Unterstützung durch Eltern, Schulfreunde und Lehrkräfte auf die Leistungsbereitschaft. Als Folge erwarten sie, dass überwiegend Schülerinnen und Schüler aus bildungsfernen Haushalten mit schulischen Defiziten aus der Krise gehen werden. Die Studie startete im Herbst 2019, wurde aber auch während und nach den ersten Schulschließungen vom 24.03. bis zum 03.07.2020 durchgeführt und im Herbst desselben Jahres wiederholt (16.11. bis 21.12.2020). Insgesamt wurden im Herbst 2.849 Schülerinnen und Schüler der gymnasialen Oberstufe in 195 Schulen aus 8 Bundesländern befragt, im Frühjahr nahmen davon erneut 1.079 der Schülerinnen und Schüler teil.

\subsection{Realer und wahrgenommener Lernerfolg}

Es stellt sich natürlich die Frage, inwiefern die Lernzeiteinbußen den Lernerfolg verringern. Dabei muss berücksichtigt werden, dass die kürzere Lernzeit mit veränderten Lernformen einhergeht, also Interaktionszeit im Unterricht entfällt, wodurch die Ermittlung des Lernrückstandes nicht linear dem Rückgang der Lernzeit entspricht. In verschiedenen nationalen wie internationalen Studien sollen Lernrückstände, die durch die Schulschließungen entstanden sein könnten, ermittelt werden.

In der sogenannten „Oxford-Studie“ konnten für die Niederlande die Leistungen von Grundschülerinnen und -schülern im Alter zwischen 8 und 11 Jahren aus jährlich stattfindenden Tests für die Jahre 2017 bis 2020 verglichen werden, wobei die Tests 2020 nach dem ersten achtwöchigen Lockdown stattfanden (Engzell et al. 2021). Dabei zeigte sich, dass die Prüfungen in Mathe, Schreiben und Lesen nach dem Lockdown deutlich schlechter ausfielen als in den drei Jahren zuvor. Die Größenordnung der Leistungseinbußen nach dem Lockdown entsprach dem Fünftel eines Schuljahres, was der Länge des Lockdowns entspricht. Bei Kindern, deren Eltern einen höheren Bildungsstand hatten, fielen die Rückstände geringer aus als bei denjenigen, welche aus einem Elternhaus mit niedrigerem Bildungsstand stammten (Engzell et al. 2021, S. 7).

Auch Schult et al. (2021) fanden in einer Studie, die jährlich den Leistungsstand von Fünftklässlerinnen und -klässlern in Baden-Württemberg erhebt, einen Leistungsrückgang in den Fächern Deutsch und Mathematik im Vergleich zum Niveau vor 
der Corona-Pandemie, ebenfalls vor allem bei Kindern aus sozial benachteiligten Lagen. Depping et al. (2021) kommen dagegen für die Hamburger Schulen zu dem Ergebnis, dass die Lerneinbußen während des ersten Lockdowns relativ gering ausgefallen sind.

Nach der Meinung von Lehrerinnen und Lehrern, die Anfang April 2020 für das deutsche Schulbarometer befragt wurden, haben die pandemiebedingten Maßnahmen seit März 2020 zu messbaren Lernrückständen bei ihren Schülerinnen und Schülern geführt (Forsa 2020b). Insgesamt 36 \% waren der Meinung, dass die Schulschließung während des ersten Lockdowns bei ihren Schülerinnen und Schülern zu deutlichen Lernrückständen führen würde. Unterschiede in der Einschätzung zeigten sich dabei zwischen den Schulformen: Während von den Lehrkräften an Gymnasien 31 \% bei mehr als der Hälfte ihrer Schülerinnen und Schüler messbare Lernrückstände erwarteten, waren dies von den Lehrkräften an Förderschulen 49 \%. Bei einer erneuten Befragung Anfang Dezember 2020 (Forsa 2020c) wurden die Lehrer und Lehrerinnen wieder um eine Einschätzung der Lernrückstände der Kinder gebeten. Insgesamt $11 \%$ waren der Meinung, dass die pandemiebedingten Maßnahmen bei (fast) allen Schülerinnen und Schülern zu messbaren Lernrückständen geführt hätten und nochmals 27 \% vermuteten Lernrückstände bei mehr als der Hälfte der Schülerinnen und Schüler (Forsa 2020c, S. 35).

Zu nennen ist in diesem Zusammenhang auch die JIMplus Corona-Studie des medienpädagogischen Forschungsverbundes Südwest, für die vom 2. bis zum 6 . April 2020 insgesamt 1.002 Schülerinnen und Schüler zwischen 12 und 19 Jahren befragt wurden. Die Heranwachsenden sollten benoten, „wie gut Schule zuhause klappt“, und vergaben eine Durchschnittsnote von 2,5 (in Schulnoten von 1 bis 6). Über die Hälfte ( $52 \%$ ) vergab eine 1 oder 2, vor allem die Jüngeren, d. h. die 12- bis 13-Jährigen, waren überwiegend (58 \%) zufrieden mit der Lernsituation zuhause. Allerdings vergaben auch $16 \%$ eine 5 oder $6, \mathrm{~d}$. h. für sie hat das Lernen zuhause überhaupt nicht funktioniert (Rathgeb 2020). Der evangelische Familienverband eaf nennt als wichtigen Faktor bei Distanzunterricht den persönlichen, bilateralen Kontakt des Lehrpersonals mit seinen Schülerinnen und Schülern, der von der Klassenlehrerin oder dem Klassenlehrer mindestens einmal wöchentlich bspw. telefonisch erfolgen sollte (Wuttke et al. 2020).

Zu den Auswirkungen des zweiten, wesentlich längeren Lockdowns liegen noch keine empirisch belastbaren Erkenntnisse zu Lernverlusten oder -rückständen vor. Die Präsidentin der Kultusministerkonferenz Britta Ernst etwa hält Lernstandser- 
hebungen erst nach den Sommerferien 2021 für sinnvoll, da sie (zum Zeitpunkt des Interviews) auch im Sommer 2021 noch mit gelegentlichem Wechselunterricht rechnete und vor allem den Wissensverlust über die Sommerferien mit abbilden wollte, um gezielte Förderangebote machen zu können (Das deutsche Schulportal 2021). Allerdings bestätigt der Blick in Nachbarländer die Tendenzen, die sich bereits beim ersten Lockdown zeigten. Für die Schweiz zeigt sich in einer Studie (Tomasik et al. 2020), die direkt vor und während des ersten Lockdowns durchgeführt wurde, dass Schülerinnen und Schüler der Sekundarstufe relativ wenig Lernrückstände zu verzeichnen hatten, dass sich aber gleichzeitig der Lerngewinn von Grundschülerinnen und -schülern stark verlangsamt hat und soziale Ungleichheiten aufbrachen.

Zierer (2021) kommt in einer Übersicht über mehrere internationale Studien zum Ergebnis, dass bereits der erste Lockdown im jeweiligen Land zu messbaren Lernrückständen geführt habe, findet allerdings große Unterschiede nach Schulform, Lehrendenpersönlichkeiten und weiteren kulturellen und sozialen Faktoren, z. B. waren Kinder aus sozial benachteiligten Lagen stärker betroffen. Eine weitere systematische Auswertung von internationalen Studien, u. a. aus Deutschland, den Niederlanden, der Schweiz, den USA und China, zum Thema Lernrückstände durch Schulschließungen aus dem Zeitraum 01.03.2020 bis 30.04.2021 bestätigte überwiegend negative Effekte von Schulschließungen auf die Leistungen von Schülerinnen und Schülern, die vor allem jüngere Schülerinnen und Schüler und diejenigen aus sozial schwachen Familien besonders betrafen (Hammerstein et al. 2021).

Aus anderen internationalen Studien zu wochenlangen streikbedingten Schulschließungen, z. B. in Belgien 1990 (Belot und Webbink 2010), der kanadischen Provinz Ontario (Baker 2013) oder Argentinien (Jaume und Willén 2019), konnten lebenslange Nachteile im Bereich Bildung und Einkommen für die betroffenen Kohorten festgestellt werden (Wößmann 2020). Allerdings ist die Situation nur eingeschränkt vergleichbar, da während eines Streiks das Lehrpersonal explizit ausfällt, während im Lockdown versucht wurde, den Lernstoff so gut wie möglich trotzdem zu vermitteln. Die Kernbotschaft dieser Studien lässt sich dennoch auf den Fall der Lockdowns übertragen, insofern als reduzierter Unterricht und Unterrichtsausfall zu langfristigen negativen Folgen für die Bildungs- und Berufskarriere der Schülerinnen und Schüler führen können.

Die Ungewissheit in Bildungsfragen belastet auch Schülerinnen und Schüler selbst und hier vor allem diejenigen, die kurz vor einem Abschluss stehen. Um eine Vor- 
stellung von der Betroffenheit der einzelnen Schularten zu bekommen, sollen zunächst die letzten verfügbaren amtlichen Zahlen zu den Absolventinnen und Absolventen aus dem Jahr 2019 genannt werden, von denen $24 \%$ mit oder ohne Hauptschulabschluss die Schule verließen, $42 \%$ mit mittlerem Abschluss und $34 \%$ mit Abitur oder Fachhochschulreife (Statistisches Bundesamt 2020). Laut der Berufsorientierungsstudie BerO machte sich fast die Hälfte (46\%) der Abiturientinnen und Abiturienten im Frühjahr nach dem ersten Lockdown Sorgen um die Schulleistungen, im Spätherbst waren es noch $41 \%$. Um die berufliche Zukunft sorgten sich noch im Frühjahr nur 25 \% der Schülerinnen und Schüler, während es im Herbst schon 41 \% waren (Anger et al. 2021). Tatsächlich nimmt die Pandemie vor allem Schülerinnen und Schülern mit Hauptschulabschluss Perspektiven auf einen Ausbildungsplatz, da sie weniger Alternativen zur Verfügung haben als Jugendliche mit Studienberechtigung, die sich auch für ein Studium entscheiden können (Maier 2020; Anger und Sandner 2020). Eine Befragung aus dem Frühjahr 2021, mit 1.743 Jugendlichen zwischen 14 und 20 Jahren, zeigte, dass vor allem Jugendliche mit niedriger Schulbildung davon ausgingen, dass sich ihre Chancen auf einen Ausbildungsplatz durch die Corona-Pandemie verschlechtert hätten (78 \%). Über alle Schularten hinweg erwarteten 71 \% schlechtere Chancen auf einen Ausbildungsplatz wegen der Corona-Pandemie, im Vergleich zu 61 \% im Jahr 2020, während dies für einen Studienplatz nur für 23 \% im Jahr 2020 und 24 \% im Jahr 2021 galt (Barlovic et al. 2020, 2021). 


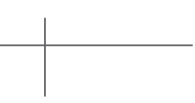

\section{Belastungen von Kindern und Jugendlichen im Lockdown}

Schul- und Kitaschließungen wurden zunächst nur unter dem Aspekt von fehlender Kinderbetreuung und Bildungsdefiziten diskutiert. Allerdings sind die Auswirkungen der Schließungen von Bildungs- und Betreuungseinrichtungen, des Wegfalls von Freizeitaktivitäten und der allgemeinen Kontaktbeschränkungen auf Kinder und Jugendliche wesentlich vielfältiger und betreffen auch mehr als nur mögliche Bildungsrückstände. Für Kinder und Jugendlichen bedeutet die Schließung von Bildungs- und Betreuungseinrichtungen auch den Verlust von sozialen Räumen, in denen Freunde getroffen werden, ein Miteinander eingeübt wird und Auseinandersetzungen unter Gleichaltrigen stattfinden können, in denen zahlreiche Anregungen wahrgenommen werden können, die Elternhaus und Schule nicht bieten.

Zunächst erfolgen eigene Analysen mit pairfam-Daten aus der Befragung in der späteren Phase des ersten Lockdowns 2020 im Vergleich zum Vorjahr, also der Zeit vor dem Ausbruch der Pandemie. Dabei differenzieren wir die Lebenssituation von Jugendlichen hinsichtlich ihrer Aktivität, ihres Stressempfindens, ihrer wahrgenommenen Einsamkeit und des Auftretens depressiver Symptome (Abschnitt 5.1).

Betrachtet man die Vielzahl an Studien, die in den letzten Monaten erschienen sind, zeigt sich, dass es - neben reduziertem Bildungserfolg - im Wesentlichen vier unterschiedliche Dimensionen von Belastungen für Kinder und Jugendliche gibt: Psychische Gesundheit, körperliche Gesundheit, Gewalterfahrung und Persönlichkeitsentwicklung. Der Forschungsstand zu diesen vier Dimensionen wird in den Abschnitten 5.2 bis 5.5 dargestellt.

Dabei muss in den folgenden Ausführungen beachtet werden, dass die Bedürfnisse von Kindern und Jugendlichen stark von ihrem Alter abhängen und sie damit auf unterschiedlichen Ebenen von der Pandemie betroffen sind. Betrachtet man entwicklungs- 
theoretische Modelle (Oerter und Montada 2008), zeigen sich in den verschiedenen Altersbereichen unterschiedliche Herausforderungen, die es zu bewältigen gibt. Diese Herausforderungen basieren auf körperlichen oder psychischen Veränderungen, gesellschaftlichen Anforderungen oder allgemeinen Werten, die angestrebt werden und können hier nur grob angerissen werden. Während für 0- bis 2-Jährige Schutz, Versorgung und enge, stabile Bindungserfahrungen wichtig sind, kommen für Kindergarten- und Vorschulkinder erste Autonomiebestrebungen und Freundschaften hinzu, die motorische und sprachliche Entwicklung wird vorangetrieben und der Umgang mit Emotionen gelernt. Das mittlere Kindesalter zwischen 6 und 11 Jahren zeichnet sich durch einen deutlich größeren Aktionsradius des Kindes und noch stärkere Autonomiebestrebungen aus, soziale Kompetenzen und Werte sind zentrale Faktoren in diesem Altersbereich. In der Vorpubertät von 12 bis 14 Jahren wird der Kontakt zu den Gleichaltrigen (Peers) immer wichtiger, die Identität wird in Auseinandersetzungen mit den Peers und der Familie entwickelt. In der Adoleszenz ab ca. 14 Jahren wird die Abgrenzung von den Eltern, zunehmende Selbstständigkeit, die Festigung der eigenen Körperidentität und das Bedürfnis nach Intimität wichtig und Beziehungen zu Gleichaltrigen stehen im Vordergrund.

All diesen Bedürfnissen stehen die Maßnahmen gegen die Pandemie diametral entgegen. Es ist fraglich, ob eine Übertragung sozialer und emotionaler Erfahrungen im virtuellen Raum im Rahmen von Distanzunterricht oder der Freizeit zu den gleichen Reifungsprozessen führt wie in der realen Welt. Für Expertinnen und Experten stellt dies eine direkte Entwicklungsbeeinträchtigung dar, und sie erwarten nachhaltige negative Folgen, sowohl psychisch als auch emotional (Baumann et al. 2021).

\subsection{Neue Analysen zur Lebenslage und psychischen Gesundheit von Jugendlichen}

Die psychische Gesundheit von Kindern und Jugendlichen kann sowohl durch die Pandemie und die damit verbundenen Sorgen um die eigene Gesundheit sowie die von Angehörigen als auch durch die Bedingungen eines Lockdowns beeinträchtigt werden. Durch Schulschließungen brechen alltagssichernde Strukturen weg, Kinder bewegen sich weniger, ernähren sich häufig ungesünder und die Mediennutzung steigt stark an. Eltern sind, durch die Situation zuhause, häufig selbst schwer belastet. Internationale Studien belegen, dass sich solche Belastungen übertragen und sich bereits bei Kindern vermehrt als psychische Beschwerden bemerkbar machen (Bignardi et al. 2020; Li und Zhou 2021; Crescentini et al. 2020). 
Im Folgenden werden eigene statistische Analysen speziell für Jugendliche dargestellt. Diese Analysen basieren auf den Daten der elften Welle (Release 11.0) des Beziehungsund Familienpanels pairfam (Brüderl et al. 2020) und der online durchgeführten pairfam Covid-19-Zusatzstudie (Walper et al. 2020). An der Covid-19-Studie nahmen zwischen dem 19.05. und dem 13.07.2020, 3.182 pairfam-Befragte teil, darunter mehr als 850 junge Erwachsene im Alter zwischen 16 und 19 Jahren. Dabei wurden ihnen dieselben Fragen gestellt wie in den Jahren zuvor, etwa danach, wie gestresst, überlastet, tatkräftig oder allein sie sich in den letzten vier Wochen überwiegend gefühlt haben, so dass man einen Vergleich von vor der Pandemie und dem Ende der ersten Lockdownphase ziehen kann. Die Fragen werden mit Hilfe einer 5-stufigen Antwortskala beantwortet, die von 1 (trifft überhaupt nicht zu) bis 5 (trifft voll und ganz zu) reicht. Einschränkend soll hier darauf hingewiesen werden, dass die Antworten Selbsteinschätzungen darstellen. Nichtsdestotrotz sind die subjektiven Daten eine wichtige Annäherung an die Themenstellung. Durch den Vergleich der Antworten vor und während der Pandemie, im Besonderen gegen Ende des ersten Lockdowns kann die Lebenssituation der Befragten direkt verglichen werden.Unsere Analysen zeigen, dass im Vergleich zu der Zeit vor der Pandemie ein signifikanter Rückgang der subjektiv wahrgenommenen Aktivität bei jungen Erwachsenen im Alter von 16 bis 19 Jahren konstatiert werden kann. Das heißt, die Jugendlichen fühlten sich im Mai und Juni 2020 deutlich weniger tatkräftig, aktiv und waren weniger energiegeladen als noch im Vorjahr (Abb. 5). Zugleich waren die Befragten weniger gestresst, überlastet und hatten auch ein geringeres Gefühl, unter Druck zu stehen. Die Unterschiede zu der Zeit vor der

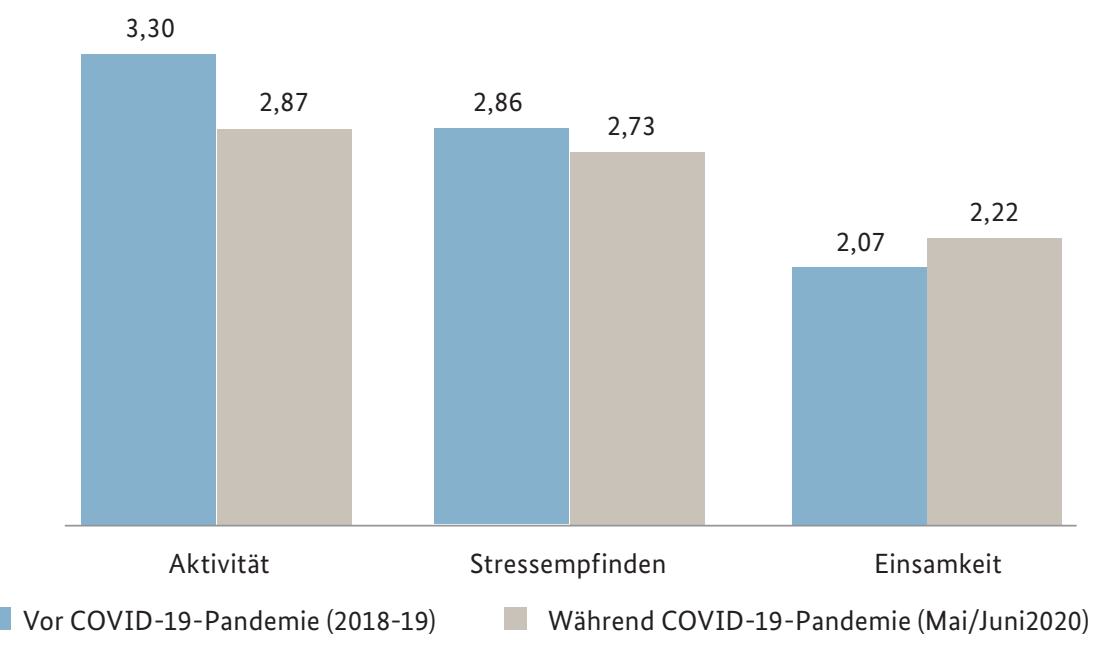

Abbildung 5: Veränderung subjektiv wahrgenommener Aktivität, Stress und Einsamkeit bei jungen Erwachsenen (5er-Skala, 1 = trifft überhaupt nicht zu, 5 = trifft voll und ganz zu)

Eigene Analysen. Datenquelle: pairfam Release 11.0 und pairfam Covid-19-Survey. 
Corona-Pandemie sind hier allerdings nur sehr gering. Auch muss beachtet werden, dass sich die Befragung nicht auf die strikte erste Lockdownphase von Mitte März bis Ende April 2020 bezieht, sondern auf die beiden folgenden Monate. Ferner kommen wir zu dem Ergebnis, dass die 16- bis 19-Jährigen sich während der Zeit der Schulschließungen und restriktiven Kontaktbeschränkungen etwas öfter allein fühlten als zuvor.

Diese Ergebnisse ziehen die Frage nach sich, wie stark die Kontaktbeschränkungen junge Menschen bereits im Frühjahr 2020 psychisch belastet haben. Denn generell gelten sowohl (sportliche) Aktivität als auch soziale Kontakte und Unterstützung als wichtige Ressourcen in der Vorbeugung mancher psychischer Erkrankung, insbesondere Depressivität (Timmons et al. 2012; Rebar et al. 2015).

Zur Messung von Depressivität verwenden wir eine in der Psychologie anerkannte Kurzskala (State and Trait Depression Scale - STDS, Spaderna et al. 2002), mit der die Jugendlichen angeben sollten, wie häufig sie verschiedene Gefühle (wie zum Beispiel negative Gefühle wie Verzweiflung oder Schwermut bzw. positive Gefühle wie Glück und Gelassenheit) während der Zeit des ersten Lockdowns erlebt haben. Die Antwortmöglichkeiten reichen von 1 (fast nie) bis 4 (fast immer). Die Auswertungen für die Teilfragen zeigen bereits, dass im Durchschnitt sich 9 der 10 Indikatoren verschlechtert haben, zum Teil deutlich (Abb. 6).

Für die Ermittlung des klinisch relevanten Depressivitätswerts jedes beziehungsweise jeder Jugendlichen wird das Ausmaß depressiver Gefühle für die 10 Fragen insgesamt mit Punkten bewertet, wobei 10 Punkte fast gar keine depressiven Gefühle beschreiben und 40 Punkte bedeuten, dass die Jugendlichen fast ausschließlich von depressiven Gefühlen berichten. Ein Depressivitätswert von mehr als 25 Punkten gilt als Grenzwert, ab dem man von klinisch relevanten depressiven Symptomen ausgehen kann (Lehr et al. 2008).

Während der Blick auf den Mittelwert der Depressivitätsskala kaum Unterschiede vor und während der Pandemie zeigt, sieht man hinsichtlich des kritischen Wertes für relevante depressive Symptomatik einen deutlichen Anstieg. Vor dem Lockdown, im Jahr 2018/19, hatten 10,2 \% der Jugendlichen klinisch relevante, depressive Symptome. Das entspricht Schätzungen zur Prävalenz von depressiven Symptomen aus anderen Studien (Polanczyk et al. 2015). Im Frühjahr 2020 steigt dieser Anteil auf 25,2 \% an (Abb. 7). In anderen Worten: Bereits gegen Ende des ersten Lockdowns weist in Deutschland jeder vierte junge Mensch zwischen 16 und 19 Jahren klinisch relevante Symptome einer Depression auf. Das ist eine statistisch signifikante Zunahme um 15 Prozentpunkte, deutlich mehr als eine Verdopplung. 
Bemerkenswert sind die Unterschiede zwischen männlichen und weiblichen Jugendlichen: Bei den männlichen Jugendlichen war der Depressivitätswert vor der Pandemie bereits geringer mit $7 \%$ im Vergleich zu $13 \%$ bei den weiblichen Jugendlichen. Dieser Unterschied hat in der Pandemie noch erheblich zugenommen: Bei den männlichen Jugendlichen hat er sich von 7 auf $15 \%$ etwa verdoppelt, bei den weiblichen Jugendlichen von 13 auf $35 \%$ sogar verdreifacht (Abb. 7). Dadurch weisen nach dem ersten Lockdown $35 \%$ der weiblichen und $15 \%$ der männlichen Jugendlichen klinisch relevante depressive Symptome auf.

Dass es keine statistisch bedeutsamen Unterschiede der Mittelwerte vor und während der Corona-Pandemie bei den Jugendlichen hinsichtlich depressiver Symptomatik gibt, kann dadurch erklärt werden, dass nicht alle Jugendlichen den Lockdown und die damit verbundenen Beschränkungen in vielen Lebensbereichen ausschließlich negativ empfunden haben. Für manche Jugendliche können beispielsweise die Schulschließungen dazu

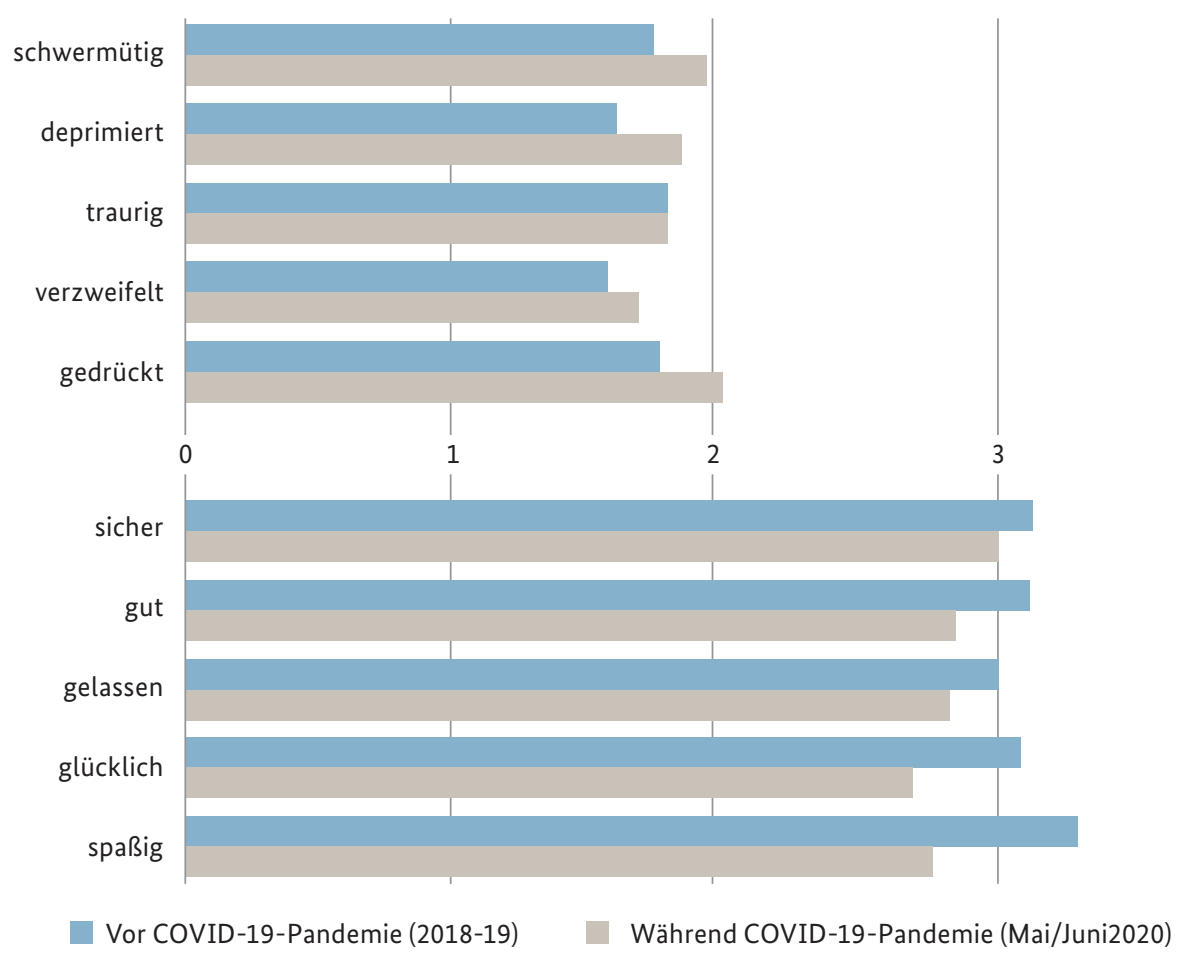

Abbildung 6: Die zehn Dimensionen der Depressivitätsskala

Eigene Analysen. Datenquelle: pairfam Release 11.0 und pairfam Covid-19-Survey. 
geführt haben, dass die sozialen Belastungen vor Ort oder der Leistungsdruck deutlich abgenommen haben und sie dadurch im Mai und Juni 2020 deutlich weniger häufig negative Gefühle angegeben haben als vor der Pandemie. Durch diesen Punkt soll jedoch nicht die Bedeutsamkeit des Anstiegs der klinisch relevanten Symptomatik von $10 \%$ auf $25 \%$ relativiert werden. Wenn ein Viertel der Jugendlichen von so starker psychischer Belastung berichtet, sollte diese Altersgruppe stärker in den Blick genommen werden.

Um die Risikogruppen besser identifizieren zu können, beschränken wir uns im Folgenden auf diejenigen Jugendlichen, die bei der Befragung im Jahr 2018/19 keine klinisch relevanten depressiven Symptome aufwiesen. Mit einer logistischen Regression schätzen wir nachfolgend, welche Jugendlichen ein erhöhtes Risiko haben, während der Pandemie depressive Symptome zu entwickeln.

Die multivariate Auswertung bestätigt die gezeigten großen Differenzen zwischen den Geschlechtern (Abb. 8). Die Wahrscheinlichkeit, dass weibliche Jugendliche depressive Symptome entwickeln, ist demnach knapp dreimal so hoch wie bei männlichen Jugendlichen. Der Migrationshintergrund scheint ein ähnlich starker Risikofaktor zu sein, und Jugendliche mit Migrationshintergrund zeigen im Vergleich zu Jugendlichen ohne Migrationshintergrund eine besonders starke Zunahme an depressiven Symptomen.

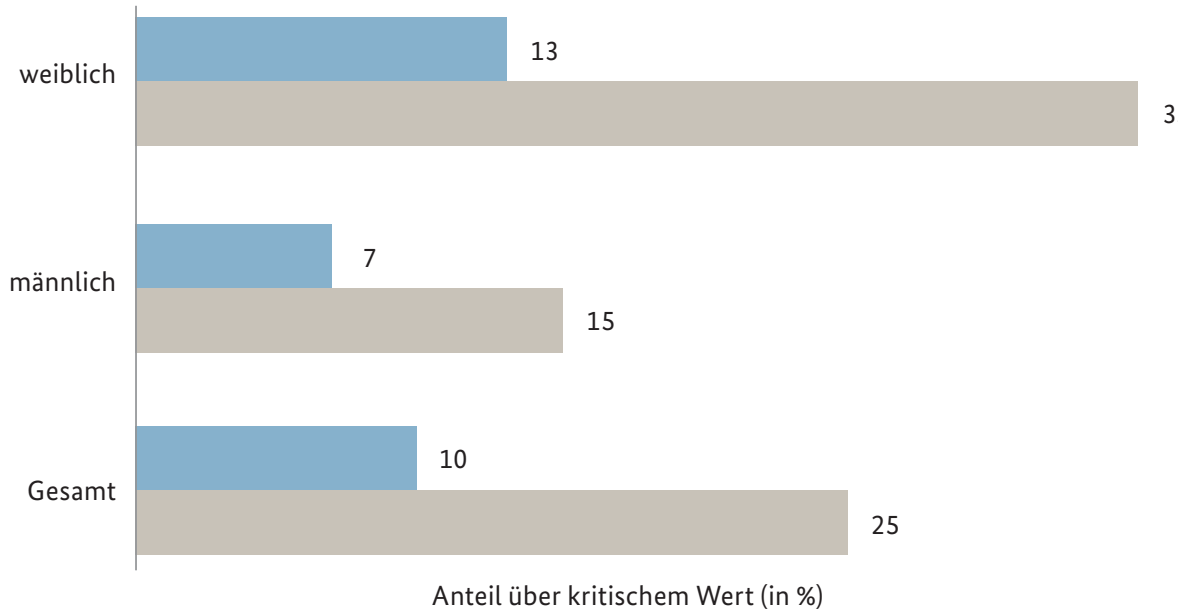

Vor COVID-19-Pandemie (2018-19)

Während COVID-19-Pandemie (Mai/Juni2020)

Abbildung 7: Auftreten von klinisch relevanten depressiven Symptomen bei 16- bis 19-Jährigen vor und in der Endphase des ersten Lockdowns in Deutschland

Eigene Analysen. Datenquelle: pairfam Release 11.0 und pairfam Covid-19-Survey. 
Während bei Jugendlichen ohne Migrationshintergrund der Anteil mit depressiven Symptomen von $9 \%$ auf $21 \%$ angestiegen ist, verdreifachte sich der Anteil bei Jugendlichen mit Migrationshintergrund von $11 \%$ auf $33 \%$. Das Risiko für depressive Symptome ist also bei Jugendlichen mit Migrationshintergrund deutlich höher als bei Jugendlichen ohne Migrationshintergrund. Das Bildungsniveau der Eltern, ob die Befragten Schüler bzw. Schülerinnen, Studierende oder Auszubildende sind, auf dem Land, in der Stadt, in Ost- oder in Westdeutschland leben, zeigt hingegen keinen Zusammenhang mit dem Risiko, depressive Symptome während des ersten Lockdowns zu entwickeln. Die Wahrscheinlichkeit, depressive Symptome zu entwickeln, ist jedoch deutlich geringer, wenn die Befragten noch bei den Eltern wohnen. Zur Kontrolle weiterer Risikofaktoren

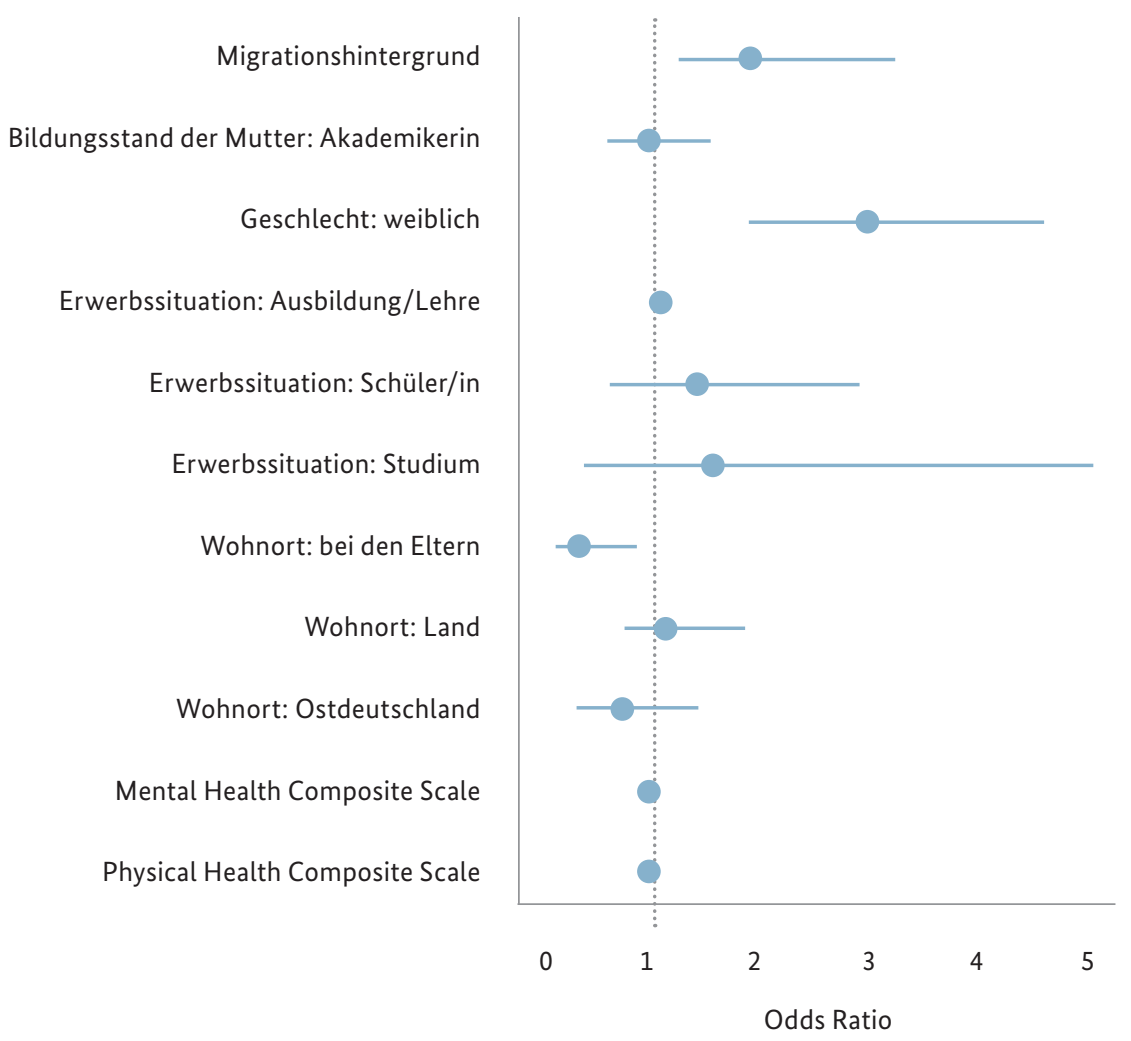

Abbildung 8: Wahrscheinlichkeit, depressive Symptome zu entwickeln, im Vergleich zum Jahr vor dem ersten Lockdown - logistische Regression (gewichtet)

Eigene Analysen. Datenquelle: pairfam Release 11.0 und pairfam Covid-19-Survey.

Anmerkung: Odd Ratios bedeuten, dass Werte über 1 eine erhöhte Chance im Vergleich zur Referenzkategorie und Werte unter 1 eine niedrigere Chance darstellen. 
wurde auch der physische und psychische Gesundheitszustand der Befragten vor der Pandemie herangezogen. Wie zu erwarten scheint sowohl ein guter psychischer als auch physischer Gesundheitszustand (vor der Pandemie) das Risiko, depressive Symptome während des ersten Lockdowns zu entwickeln, zu schmälern. Dies lässt vermuten, dass eine gute körperliche und seelische Verfassung eine verbesserte Resilienz zur Folge hat.

\subsection{Psychische Gesundheit: Forschungsüberblick}

Das tatsächliche Ausmaß der Beeinträchtigungen der psychischen Gesundheit durch die Maßnahmen im Zusammenhang mit der Eindämmung der Corona-Pandemie wird sich erst zeigen, wenn die Pandemie überstanden ist. Es gibt einzelne Hinweise aus Psychiatrien sowie eine Sonderauswertung der Krankenkasse DAK, die eine hohe Steigerungsrate bei Jugendlichen in psychiatrischen Einrichtungen zeigt, dies beruht jedoch auf begrenzten Datengrundlagen mit niedrigen Fallzahlen (dpa 2021).

Für Deutschland zeigt die COPSY-Studie (COrona und PSYche) des Universitätsklinikums Hamburg-Eppendorf (UKE) belastbare empirische Befunde zu psychischen Belastungen. Sie ist als Längsschnitt angelegt und erfasst als eine der wenigen nationalen und internationalen Studien die Situation über beide Lockdowns (Ravens-Sieberer et al. 2020, 2021). Für die erste Befragung wurden vom 26.05. bis zum 10.06.2020 insgesamt 1.040 Kinder und Jugendliche zwischen 11 und 17 Jahren und 546 Eltern zu ihren 7- bis 10-jährigen Kindern befragt. Mitte Dezember 2020 bis Mitte Januar 2021 wurden nochmals mehr als 1.000 Kinder und Jugendliche und mehr als 1.600 Eltern befragt, von denen $80 \%$ bereits an der ersten Studie teilgenommen hatten, $20 \%$ wurden nacherhoben, um die Repräsentativität der Ergebnisse zu gewährleisten. Allgemein lässt sich zunächst auch für Deutschland festhalten, dass die Herausforderungen der Pandemie die Lebensqualität und das psychische Wohlbefinden von Kindern und Jugendlichen verringert und gleichzeitig das Risiko für psychische Auffälligkeiten erhöht haben.

Im Vergleich zu der Zeit vor der Pandemie haben während der Pandemie bis in den zweiten Lockdown psychosomatische Beschwerden bei Kindern und Jugendlichen zugenommen, z. B. Bauchschmerzen von $21 \%$ auf $36 \%$, Niedergeschlagenheit von $23 \%$ auf $43 \%$, Kopfschmerzen von $28 \%$ auf $46 \%$ und Gereiztheit von $40 \%$ auf $57 \%$ (Abb. 9).

Auch das Risiko für Verhaltensauffälligkeiten stieg von $13 \%$ auf 19 \% (Abb. 10), emotionale Probleme nahmen von $16 \%$ auf $24 \%$ zu, Hyperaktivität stieg zunächst von $13 \%$ auf $24 \%$ nach dem ersten Lockdown und sank dann wieder auf $20 \%$, und Probleme im 


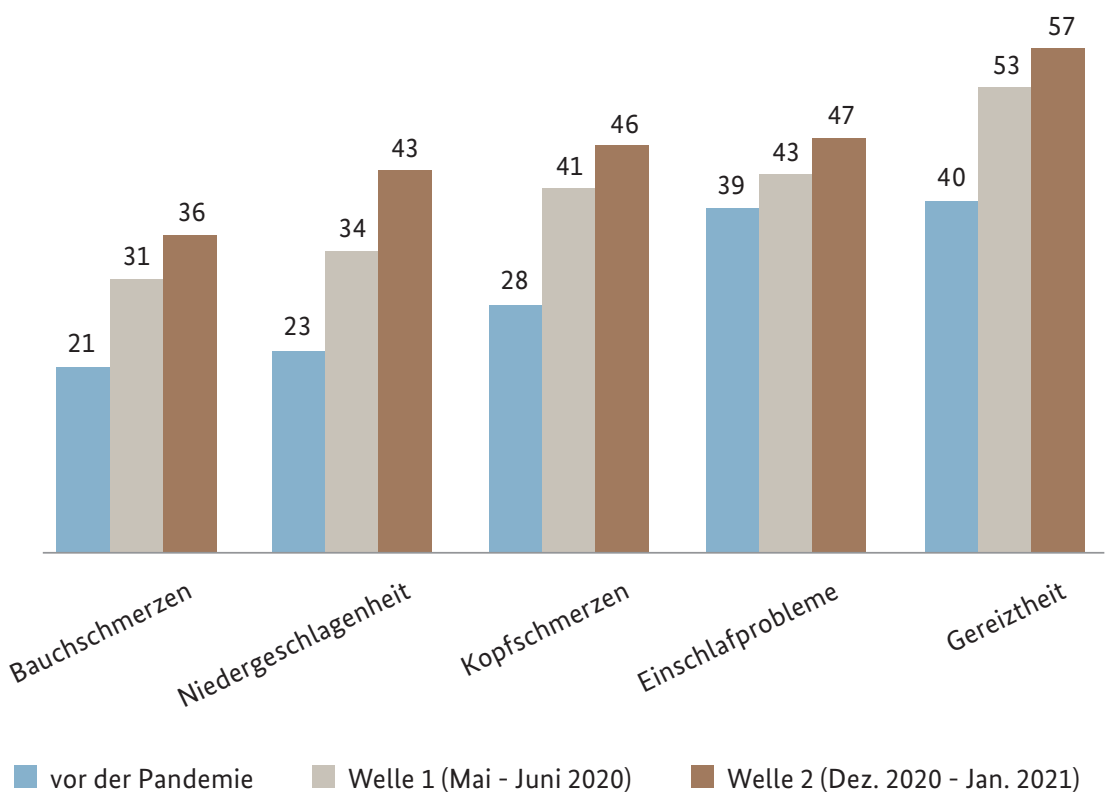

Abbildung 9: Subjektive Aussagen zu Beschwerden von Kindern nach dem ersten und während des zweiten Lockdowns (Anteil „mindestens einmal pro Woche“ in \%)

Quelle: Ravens-Sieberer et al. 2021.

sozialen Umgang mit Freunden haben von $11 \%$ auf $27 \%$ zugenommen (Ravens-Sieberer et al. 2020, 2021). Die Autorinnen und Autoren der Studie weisen darauf hin, dass es sich bei den berichteten Symptomen nicht um diagnostizierte Befunde handelt, sondern um Selbsteinschätzungen. Trotzdem können sie ein Bild über die Belastung von Kindern und Jugendlichen durch die Pandemie vermitteln, und es wird deutlich, dass sich die Symptome im zweiten Lockdown nochmals verschlimmert haben. Die Studie zeigt, dass vor allem sozial benachteiligte Kinder gefährdet sind, dass aber ein positives Familienklima, wenn bspw. Eltern ihren Kindern viel Zeit widmen und ihre Ängste und Probleme ernst nehmen, Belastungen entgegenwirkt.

Die Pandemie hat die Lebensqualität von Kindern und Jugendlichen stark verringert, dies hat sich durch den zweiten Lockdown noch verschärft. Insgesamt 71 \% der Kinder und Jugendlichen fühlten sich durch die Kontaktbeschränkungen während des ersten Lockdowns belastet, zu Beginn des zweiten Lockdowns waren es $83 \%$. Kinder und Jugendliche berichteten nicht selten von mehr Streit und dass sich das Verhältnis zu Freunden während der Kontaktbeschränkungen verschlechtert habe, was als große Belastung empfunden wurde. Etwa 45 \% fanden die Schulsituation im zweiten 

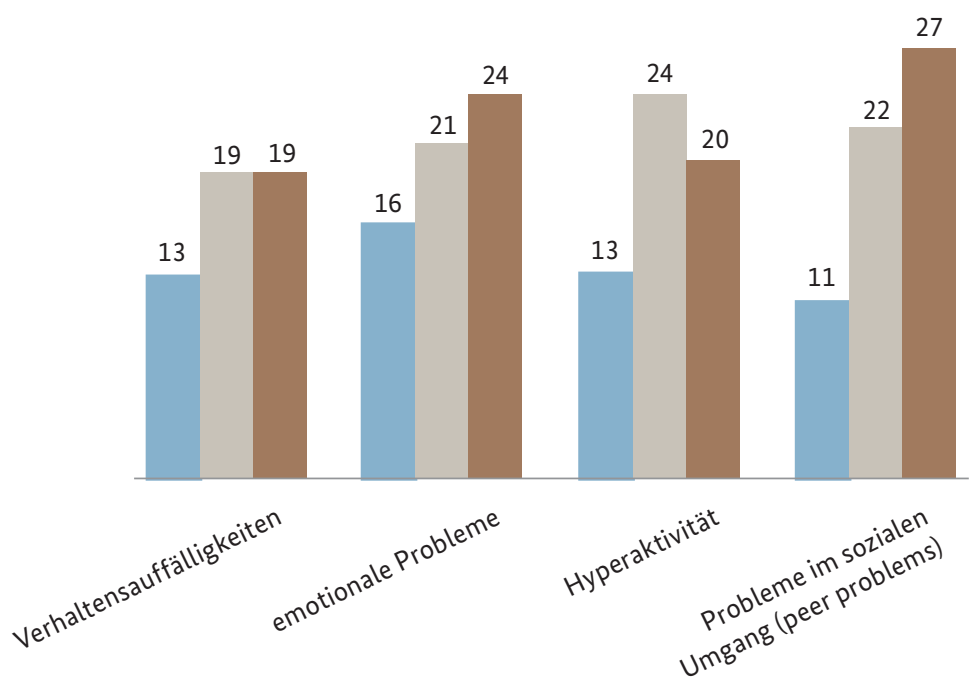

vor der Pandemie

Welle 1 (Mai - Juni 2020)

Welle 2 (Dez. 2020 - Jan. 2021)

Abbildung 10: Veränderung des Risikos für Verhaltensauffälligkeiten nach dem ersten und während des zweiten Lockdowns (Anteil „grenzwertig“ und „auffällig“ in \%)

Quelle: Ravens-Sieberer et al. 2021.

Lockdown anstrengender als im ersten Lockdown, umgekehrt nur $10 \%$ (Lemm 2021). Dabei kann es sein, dass die Belastungswerte, die zu einer späteren Phase des zweiten Lockdowns gemessen werden, noch gravierender sind. Schließlich haben auch andere negative Verhaltensweisen, wie übermäßiger Medienkonsum, ungesunde Ernährung und zu wenig körperliche Bewegung im zweiten Lockdown nochmals zugenommen (Universitätsklinikum Hamburg-Eppendorf 10.02.2021).

Dass die Schulschließungen und die damit einhergehenden Kontaktreduzierungen für Kinder sehr belastend waren, bestätigen auch andere Studien, basierend auf der Einschätzung von Eltern, die hier bereits in anderen Zusammenhängen vorgestellt wurden, wie z. B. das ifo-Bildungsbarometer (Wößmann et al. 2020a, 2021). Demnach wurden die Belastungen im zweiten Lockdown als wesentlich stärker erlebt als im ersten Lockdown. Während im Frühjahr 2020 noch 86 \% der Eltern angaben, sie wären mit der Situation gut klargekommen, waren dies im Winter 2020/21 nur noch 71 \%. Im Frühjahr 2020 empfanden $36 \%$ der Eltern den Lockdown als große psychische Belastung für ihr Kind, im Winter 2020/21 waren es $49 \%$. Für sich selbst nahmen im Frühjahr 2020 insgesamt 38 \% der Eltern den Lockdown als große psychische Belastung wahr, im Winter 2020/21 sogar 51 \%. 
Für die DJI-Studie „Kindsein in Zeiten von Corona“ wurden nach dem ersten Lockdown 8.127 Eltern mit Kindern im Alter von 3 bis 15 Jahren befragt, unter anderem zur Situation in den Familien und dem Wohlbefinden der Kinder. Von den Eltern glauben $27 \%$, dass sich ihr Kind einsam fühle und weitere $27 \%$, dass dies zumindest teilweise zuträfe. Dabei fanden sich Unterschiede zwischen Kindergartenkindern mit $31 \%$, Grundschulkindern mit $27 \%$ sowie Kindern im Sekundarstufenalter mit $18 \%$. Hier zeigen sich nach Meinung des Autorenteams unter anderem die zunehmende Bedeutung verbaler Kommunikation für ältere Kinder und die zunehmenden Möglichkeiten, selbstständig über Telefon, Skype oder andere Messengerdienste mit Freunden in Kontakt zu treten. Für jüngere Kinder ist der direkte Kontakt noch wichtiger, da sie sich eher über das gemeinsame Spiel austauschen als über verbale Kommunikation. Weitere Einflussfaktoren auf Einsamkeitsgefühle waren die Anzahl der Geschwister und auch die soziale Lage der Eltern (Langmeyer et al. 2020).

Eine weitere Gefahr des Lockdowns ist ein Anstieg eines riskanten oder pathologischen Medienkonsums von Kindern und Jugendlichen. Während des ersten Lockdowns stiegen die Nutzungszahlen von Onlinespielen um 75 \% von 79 auf 139 Minuten pro Tag. Für die Nutzung sozialer Medien erfolgte ein Anstieg um 66 \% von 116 auf 193 Minuten. Bereits im August warnten Suchtforscherinnen und - forscher vor einem zweiten Lockdown, der diese ungünstigen Entwicklungen noch weiter vorantreiben würde (Bühring 2020).

Die Folgen des Lockdowns für Kinder aus Förderschulen und Sonderschulen sind besonders belastend. Für sie ist nicht nur der Schulunterricht weggefallen, sondern auch viele Therapieangebote und häufig sind diese nach dem Ende des ersten Lockdowns auch nicht wieder aufgenommen worden, weil die Kinder durch ihre körperliche oder geistige Beeinträchtigung zu den Risikogruppen gehören. Die Entspannung, die andere Kinder und Jugendliche und ihre Familien nach dem Lockdown erfahren haben, blieb vielen dieser Familien versagt (Kugelmeier und Schmolze-Krahn 2020a, 2020b).

\subsection{Hochgerechnete Größenordnungen: Wie viele Kinder und Jugendliche weisen psychische Belastungen auf?}

Die Größenordnungen der Zunahme psychischer Belastungen und Krankheiten lässt sich nur schwer abschätzen, da die Diagnostik und deren statistische Erfassung entsprechende Zeit benötigen. Die Selbsteinschätzungen durch Eltern bzw. Jugendliche sind nicht mit einer Diagnose zu verwechseln, sie weisen jedoch auf erhebliche Größenordnungen hin. In Tabelle 2 wurden unsere eigenen Befunde zu depressiven 
Symptomen mit pairfam-Daten sowie die Befunde von Ravens-Sieberer et al. (2021) mit demografischen Daten zur Bevölkerungsgröße der entsprechenden Altersgruppen kombiniert. Der Anteil von Kindern und Jugendlichen mit depressiven Symptomen ist von 10,2 \% auf 25,2\% angestiegen. Da 3,2 Mio. Jugendliche im Alter 16 bis 19 Jahren in Deutschland leben, würde dieser Anstieg infolge der Corona-Pandemie und der Corona-Maßnahmen einer Zunahme von 477.000 Jugendlichen mit klinisch relevanten depressiven Symptomen entsprechen. Auch die Geschlechtsunterschiede lassen sich hochrechnen: Es betrifft 137.000 Jungen bzw. junge Männer und 340.000 Mädchen bzw. junge Frauen im Alter von 16 bis 19 Jahren.

Der Anstieg der Zahl an Jugendlichen (11-17 Jahre) mit mindestens einmal pro Woche Bauchschmerzen oder Einschlafproblemen wird hier nicht hochgerechnet, da dies vergleichsweise niederschwellige Kriterien sind und die Anteile vor der Pandemie bereits bei 21 bzw. 39 \% lagen. Der Anstieg von Kindern und Jugendlichen mit emo-

\begin{tabular}{cccccc}
\hline $\begin{array}{c}\text { Anteil vor der } \\
\text { Pandemie } \\
\text { (in \%) }\end{array}$ & $\begin{array}{c}\text { Anteil im 1. } \\
\text { Lockdown } \\
\text { (in \%) }\end{array}$ & $\begin{array}{c}\text { Anteil im 2. } \\
\text { Lockdown } \\
\text { (in \%) }\end{array}$ & $\begin{array}{c}\text { bezogene } \\
\text { Alters- } \\
\text { gruppe }\end{array}$ & $\begin{array}{c}\text { Bevölkerung } \\
\text { der Alters- } \\
\text { gruppe } \\
\text { (in 1.000) }\end{array}$ & $\begin{array}{c}\text { Hochrechnung } \\
\text { der Zunahme } \\
\text { an betroffenen } \\
\text { Kindern (in 1.000) }\end{array}$ \\
\hline
\end{tabular}

\begin{tabular}{|c|c|c|c|c|c|c|}
\hline \multicolumn{7}{|c|}{ Eigene Analysen (pairfam-Daten) ${ }^{1}$} \\
\hline $\begin{array}{l}\text { Klinisch relevante } \\
\text { depressive Symptome }\end{array}$ & 10,2 & 25,2 & & 16 bis 19 & $3.182,4$ & 477 \\
\hline - davon männlich & 7 & 15 & & 16 bis 19 & $1.649,5$ & 137 \\
\hline - davon weiblich & 13 & 35 & & 16 bis 19 & $1.532,9$ & 340 \\
\hline \multicolumn{7}{|l|}{ CoPsy-Studie ${ }^{2}$} \\
\hline Bauchschmerzen & 21,3 & 30,5 & 36,4 & 11 bis 17 & $5.257,2$ & * \\
\hline Einschlafprobleme & 39,2 & 43,3 & 47,4 & 11 bis 17 & $5.257,2$ & * \\
\hline Emotionale Probleme & 16,4 & 20,9 & 23,7 & 7 bis 17 & $8.201,1$ & * \\
\hline Verhaltensauffälligkeiten & 13,1 & 19,2 & 19,0 & 7 bis 17 & $8.201,1$ & * \\
\hline $\begin{array}{l}\text { Geringere gesundheits- } \\
\text { bezogene Lebensqualität }\end{array}$ & 15,3 & 40,2 & 47,7 & 11 bis 17 & $5.257,2$ & 1703 \\
\hline
\end{tabular}

Tabelle 2: Größenordnung der psychischen Belastungen bei Kindern und Jugendlichen

Datenquelle: ${ }^{1}$ Eigene Analysen mit pairfam-Daten; ${ }^{2}$ (Ravens-Sieberer et al. 2021), Statistisches Bundesamt, Bevölkerung am 31.12.2019; eigene Berechnungen; * = hochgerechnet wurden nur Anstiege, die deutlich größer sind als der Ausgangswert vor der Pandemie, also mindestens Verdoppelungen. 
tionalen Problemen und bei Verhaltensauffälligkeiten ist zwar deutlich, allerdings hat sich die Zahl der betroffenen Kinder in der Pandemie weniger als verdoppelt. Das bedeutet, dass von den betroffenen Kindern mehr als die Hälfte auch ohne Pandemie diese Ausprägung hat. Mehr als verdoppelt hat sich dagegen der Anteil der Kinder mit geringer gesundheitsbezogener Lebensqualität: Dieser Anstieg entspricht einer Größenordnung von 1,7 Mio. Kindern und Jugendlichen im Alter von 11 bis 17 Jahren.

Diese Größenordnungen sind vorläufig und basieren auf Selbsteinschätzungen und nicht auf Diagnosen sowie auf zahlenmäßig begrenzten Stichproben und nicht auf Vollerhebungen; sie sollen hier nur zeigen, dass es sich um eine erhebliche Anzahl von betroffenen Kindern und Jugendlichen handelt. Weitere Forschung zum Ausmaß psychischer Erkrankungen infolge der Corona-Krise auf Basis von Diagnosen und repräsentativen Daten ist dringend notwendig.

\subsection{Körperliche Gesundheit}

Neben der psychischen Belastung wirken sich die Schulschließungen auch auf die körperliche Gesundheit von Kindern aus, vor allem auf die tägliche Bewegung und die Ernährung. Dabei schlagen nicht nur die fehlenden Möglichkeiten aufgrund von Schließungen von Sportstätten oder Schulmensen zu Buche, sondern auch die Tatsache, dass gesundes Verhalten während strukturierter Tagesabläufe, wie sie der Schulalltag vorgibt, leichter eingehalten wird. Solche Strukturen können aber während eines Lockdowns von Eltern und Kindern selbst nicht immer aufrechterhalten werden (Brazendale et al. 2017).

\section{BEWEGUNG}

Kinder erreichen ihr tägliches Pensum an Bewegung normalerweise nicht zuhause, wo mehr Zeit mit Erholung verbracht wird, sondern auf dem Schulweg, beim Schulsport, bei sonstigem Vereinssport oder auf dem Spielplatz (Guan et al. 2020). Während des ersten Lockdowns fielen die meisten dieser Möglichkeiten weg. Es war zeitweilig verboten, auf Spielplätzen zu spielen und auch Schulsport fand nicht statt und wurde auch nach der Aufhebung des ersten Lockdowns aus Gründen der Ansteckungsgefahr überhaupt nicht, spät oder in sehr reduzierter Form wiederaufgenommen. Das außerschulische Sportangebot war gleichermaßen betroffen, so mussten die Sportvereine schließen, konnten ihr Programm nach dem ersten Lockdown nur sehr begrenzt wieder anbieten und wurden im zweiten Lockdown 
wieder geschlossen. Nimmt man die Mitgliedschaften des Deutschen Olympischen Sportbundes als Grundlage, betrifft dies fast 80 \% der Jungen und 60 \% der Mädchen im Alter zwischen 7 und 14 Jahren (Abb. 11). Doch schon von den unter 7-Jährigen sind mindestens ein Viertel der Kinder in einem Sportverein aktiv, in der Altersgruppe 15 bis 18 fast zwei Drittel der Jungen und fast die Hälfte der Mädchen (Deutscher Olympischer Sportbund 2021). Zwar waren die Mitgliederverluste im Pandemiejahr 2020, die bis Oktober 2020 mit teils vorläufigen Zahlen auf durchschnittlich 2,3 \% geschätzt werden, eher gering, jedoch fehlten gerade bei den Kindern und Jugendlichen die Neuanmeldungen (Rieger 2021).

Internationale Studien weisen darauf hin, dass während eines Lockdowns der Anteil der täglichen Bewegungszeit bei Kindern und Jugendlichen zugunsten von Medienzeit abnimmt (Schmidt et al. 2020; Moore et al. 2020). Für Deutschland bietet die Motorik-Modul-Studie (MoMo) des Karlsruher Instituts für Technologie (KIT) und der Pädagogischen Hochschule Karlsruhe (PHKA) die Möglichkeit, zu vergleichen, wie sich das Verhalten von Kindern und Jugendlichen in Bezug auf Sport und sonstige körperliche Aktivitäten während des ersten Lockdowns verändert hat. Der Vorteil der Studie ist, dass sie als Panelstudie angelegt ist und die ersten Befragungen bereits vor dem ersten Lockdown stattfanden. Zunächst bestätigen sich die Ergebnisse aus den

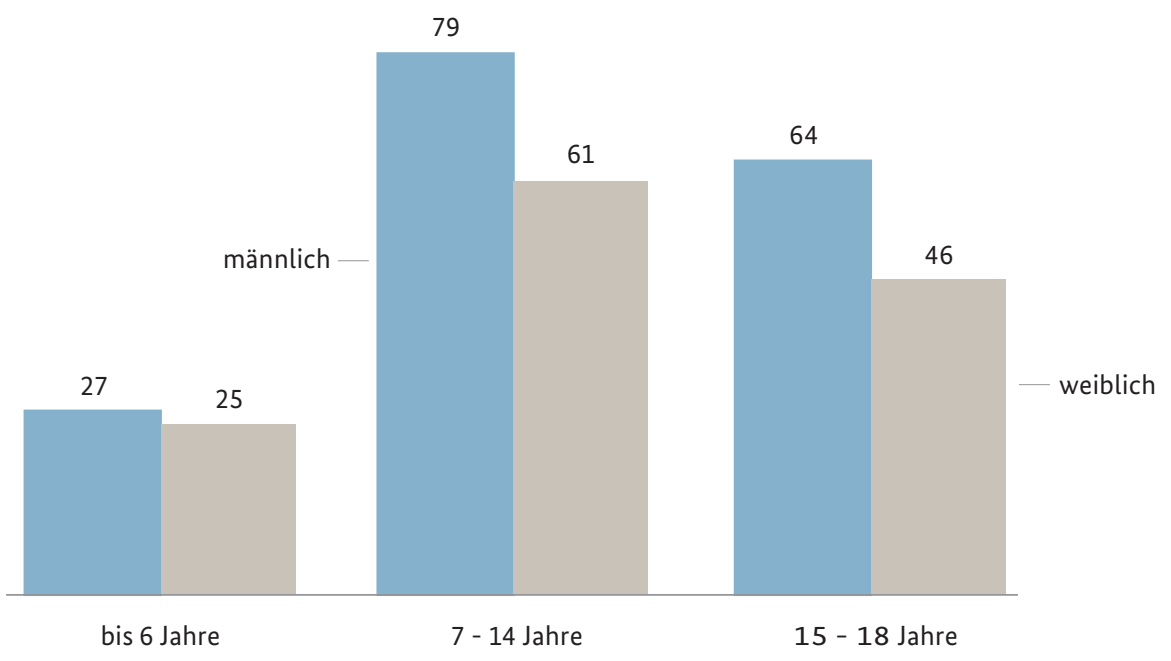

Abbildung 11: Mitgliedschaft in Sportvereinen, in Anteilen an der jeweiligen Bevölkerungsgruppe (Anteile in \%)

Quelle: Deutscher Olympischer Sportbund 2021. 
Bildungsstudien: Die Bildschirmzeit nahm um 60 Minuten täglich zu. Was Bewegung angeht, fand, wie zu erwarten, aufgrund der Einschränkungen der faktischen Möglichkeiten organisierter Sport praktisch nicht statt, allerdings nahm nicht organisierter Sport, wie z. B. Kicken, Basketball- oder Federballspielen, von durchschnittlich rund 7 auf 24 Minuten täglich zu. Insofern ging die Zeit, die täglich insgesamt mit Sport verbracht wurde, nur um durchschnittlich 11 Minuten täglich zurück. Auch die Zeit für Alltagsaktivitäten, wie z. B. draußen spielen, Spazierengehen und Fahrradfahren, nahm während des ersten Lockdowns zu, so dass Kinder und Jugendliche durchschnittlich 36 Minuten mehr aktiv bewegte Zeit pro Tag hatten als vor dem Lockdown (Abb. 12). Allerdings haben Alltagsaktivitäten nicht die gleiche Intensität wie sportliches Training und Wettkämpfe. Zudem fallen ohne Verein und Schule soziale Aspekte des organisierten Sports weg, die durch Individualsport nicht ausgeglichen werden können. Die Autorinnen und Autoren der Studie äußerten sich im Hinblick auf den zweiten Lockdown im Dezember auch skeptisch, ob sich in der kalten Jahreszeit das Bewegungsniveau aufrechterhalten lasse (Karlsruher Institut für Technologie 11.12.2020). Für zukünftige Lockdowns folgern sie, dass die Politik allen Kindern und Jugendlichen Zugang zu nicht organisierten Bewegungsmöglichkeiten bieten sollte, speziell für Jugendliche wird der Ausbau von Online-Angeboten vorgeschlagen (Schmidt et al. 2020).

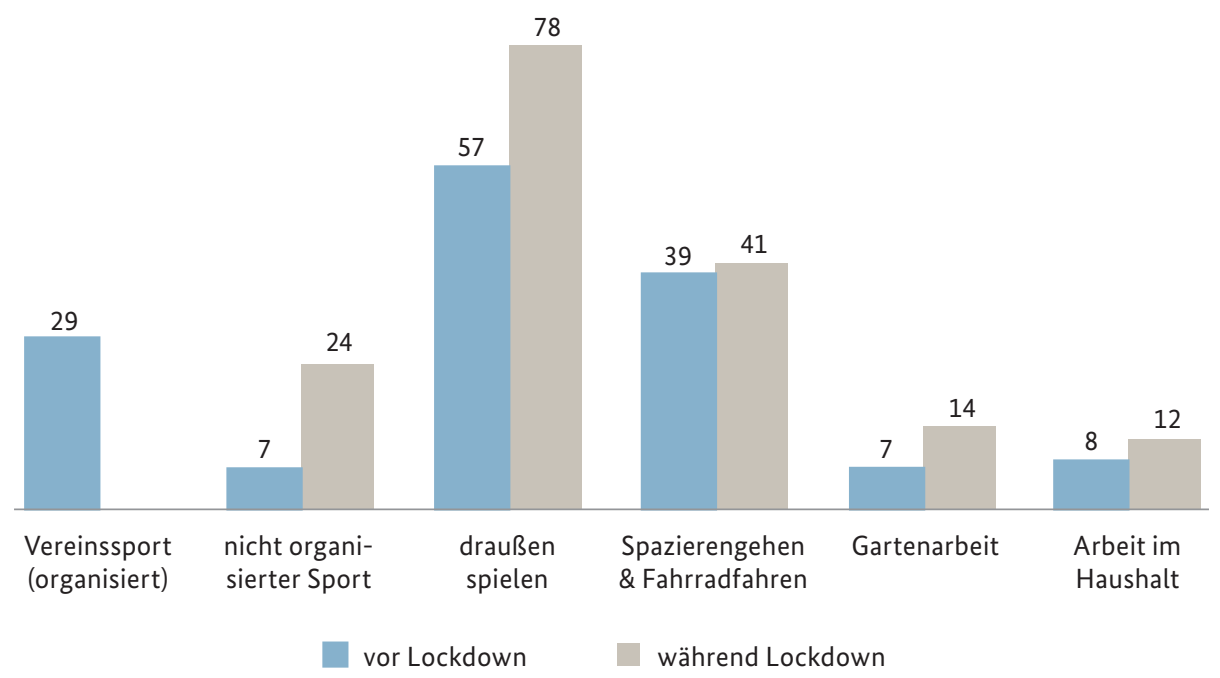

Abbildung 12: Aktivitäten von 4- bis 17-Jährigen vor und während des ersten Lockdowns in Deutschland (in Minuten pro Tag)

Quelle: Schmidt et al. 2020. 
Ergänzend ist anzumerken, dass Expertinnen und Experten darauf hinweisen, dass die Wahrscheinlichkeit von sportlicher Betätigung während eines Lockdowns auch vom sportlichen Aktivitätsniveau vorher abhängt. Ebenso spielt der soziale Kontext eine entscheidende Rolle, d. h. mit höherer Bildung der Eltern steigt auch die Wahrscheinlichkeit von körperlicher Betätigung der Kinder. Zudem setzt die Nutzung von Online-Angeboten zuerst das Vorhandensein entsprechender Endgeräte voraus (KauerBerk et al. 2020). Auch die Wohnumgebung ist von Bedeutung, insofern als Einfamilienhäuser mit Garten schlicht mehr Möglichkeiten bieten, sich zu bewegen als Wohnungen im Hochhaus. Und schließlich fördern Geschwisterkinder und Haustiere die Wahrscheinlichkeit, dass Kinder und Jugendliche sich mehr bewegen (Schneider et al. 2020; Moore et al. 2020).

Zu den Sportstätten, die geschlossen wurden und auch im Sommer 2020 meist nur unter Auflagen für wenige Besucherinnen und Besucher wieder geöffnet wurden, gehören auch Schwimmbäder. Bereits vor dem Lockdown hat die Deutsche LebensRettungs-Gesellschaft e.V. angemahnt, dass fast $60 \%$ der Zehnjährigen keine sicheren Schwimmerinnen bzw. Schwimmer seien und 2017 nur durchschnittlich $40 \%$ der 6- bis 10-Jährigen ein Jugendschwimmabzeichen besaßen. Gleichzeitig würden immer mehr Schwimmbäder geschlossen. Insofern verstärkt ein Lockdown, in dem sowohl Schwimmkurse in öffentlichen Bädern wie auch Schwimmen als Schulsport wegfällt, die Tendenz zu mehr Nichtschwimmerinnen und -schwimmern unter Kindern und Jugendlichen (Deutsche Lebens-Rettungs-Gesellschaft 2017), was eindeutig den Beschlüssen der Kultusministerkonferenz von 2017 entgegensteht, die besagen, dass jedes Kind schwimmen lernen sollte (Kultusministerkonferenz 2017).

\section{ERNÄHRUNG}

Zu einer gesunden Lebensweise gehört auch eine entsprechende Ernährung. Dafür ließen Expertinnen und Experten des Else Kröner-Fresenius-Zentrum für Ernährungsmedizin (EKFZ) die Forsa-Studie „Lebensstil und Ernährung in Corona-Zeiten“ durchführen, für die vom 11. bis zum 16.09.2020 1.000 Eltern von Kindern bis 14 Jahren befragt wurden. Der erste Lockdown im Frühjahr 2020 bedeutete in dieser Hinsicht, dass in den Familien häufiger selbst gekocht wurde, da etwa zwei Drittel der befragten Eltern diese Phase zumindest teilweise im Homeoffice verbracht haben. Der Verzehr von To-Go-Mahlzeiten und die Nutzung von Lieferdiensten haben sich dagegen wenig verändert. Da nur nach Veränderungen gefragt wurde, können an dieser Stelle keine Angaben zum tatsächlichen Umfang gemacht werden, in dem diese Angebote genutzt werden. 
Zunächst bestätigen die Ergebnisse andere Studien in der Hinsicht, dass 38 \% der Eltern angaben, ihr Kind habe sich während des Lockdowns weniger bewegt. Gleichzeitig hat sich das Risiko für Übergewicht und Fehlernährung bei den Kindern erhöht. Rund $10 \%$ der Kinder haben während des ersten Lockdowns zugenommen, vor allem 10- bis 14-Jährige und hier wiederum vor allem Jungen. Zudem wurde zwar mehr Obst gegessen (20\%), aber auch mehr Süßigkeiten (20\%), Salzgebäck (18\%) und Softdrinks (18\%) konsumiert. In der Studie zeigte sich ein Zusammenhang zwischen der Bildung der Eltern und der Häufigkeit von Homeoffice im Lockdown. Die Arbeit im Homeoffice begünstigt, dass Eltern Mahlzeiten häufiger selbst kochen, die zumeist auch gesünder sind, und wirkt darüber einer möglichen Gewichtszunahme der Kinder entgegen. Kinder von Eltern ohne akademische Bildung, die seltener im Homeoffice arbeiten können, hatten ein 2,5-fach höheres Risiko zuzunehmen als Kinder von Eltern mit akademischer Bildung (Koletzko et al. 2021).

\subsection{Gewalterfahrung}

Der Alltag von Kindern in der Pandemie, nochmals verstärkt durch einen Lockdown, ist gekennzeichnet durch einen Mangel an außerfamiliären Räumen sowie wenig bis keinen Kontakt zu Freunden bzw. Freundinnen und Peers. Dadurch sind Familien wesentlich stärker auf sich selbst gestellt, so dass schwelende Konflikte vermehrt sichtbar werden und aufbrechen. Gleichzeitig sind viele Eltern gestresster, denn sie müssen Kinderbetreuung und -beschulung mit Erwerbsarbeit, eventuell Sorgen um den Arbeitsplatz und der eigenen Angst vor den Folgen der Pandemie in Einklang bringen. Viele Hilfsangebote - und damit Ausweichmöglichkeiten und Kontrollmechanismen - fallen aus Gründen des Infektionsschutzes weg. Erfahrungen aus wirtschaftlichen Krisen der letzten Jahre zeigen, dass es in solchen Situationen immer zu einem deutlichen Anstieg an körperlicher, emotionaler und sexualisierter Gewalt an Kindern kam (Fegert et al. 2020; Schneider et al. 2017). Internationale Studien bekräftigen diese Vermutung für die Corona-Pandemie, so haben Lee et al. (2021) für die USA einen Zusammenhang zwischen krisenbedingten wirtschaftlichen Schwierigkeiten, sozialer Isolation und Kindesmisshandlung in der Pandemie nachgewiesen.

Für die Zeit des ersten Lockdowns wurde von der Technischen Universität München und dem Leibniz-Institut für Wirtschaftsforschung eine Studie mit 3.800 Frauen durchgeführt, die über ihre Erfahrungen während des ersten Lockdowns berichtet haben. Von innen erfuhren 3,1 \% körperliche Gewalt, 3,6 \% sexuelle Gewalt und 3,8 \% emotionale Gewalt. In 6,5\% der Haushalte wurden Kinder körperlich bestraft, vor allem unter den 
extremen Bedingungen einer Quarantäne (11\%), bei finanziellen Sorgen der Eltern (10\%), bei Arbeitsplatzverlust (9\%) oder wenn einer der Eltern Angst oder Depressionen hatte (14 \%) (Technische Universität München 2020). Gewalterfahrungen gehören zu den stärksten Risikofaktoren für die psychische Gesundheit von Kindern und Jugendlichen mit langwierigen Folgen bis ins Erwachsenenalter hinein (Schlack et al. 2020). Dies gilt nicht nur für selbst erfahrene Gewalt, sondern auch bei Gewalt im familiären Umfeld, die von Kindern miterlebt wird (Clemens et al. 2021). Die Wahrscheinlichkeit, häusliche Gewalt zu erleben, hängt von verschiedenen, pandemieunabhängigen Prädiktoren ab. Baumann et al. (2021) nennen als mögliche gewaltbegünstigende Faktoren z. B. mangelnde psychische Stabilität der Erwachsenen, eine traditionalistische Rollenverteilung, evtl. mit männlichkeitsidealisierenden Wertesystemen, die Impulsivität des Kindes, die Konfliktdichte und -intensität oder Alkoholkonsum in der Familie, wobei hier kein Automatismus vorliegt und keiner dieser Faktoren zu Gewalt führen muss. Die Pandemie und damit zusammenhängende Maßnahmen begünstigen Gewalt, indem pandemiebezogene Angst als Gewaltauslöser hinzukommt. Gleichzeitig wird durch Schul- und Kitaschließungen die Entdeckungsgefahr minimiert. Eine bundesweite Vollerhebung des Deutschen Jugendinstitutes aller Jugendämter zu den Folgen der Pandemie auf die Kinder- und Jugendhilfe erbrachte zunächst keine Zunahme von Hinweisen auf mehr Kindeswohlgefährdungen, allerdings wurde davon ausgegangen, dass die Dunkelziffer angestiegen sei (Mairhofer et al. 2020). Diese Vermutung bestätigte sich, für das Jahr 2020 meldet das Statistische Bundesamt mit $9 \%$ mehr Fällen einen Höchststand an Kindeswohlgefährdungen (Statistisches Bundesamt 2021b).

\subsection{Persönlichkeitsentwicklung und Zeitgefühl}

Jugendforscherinnen und -forscher warnen vor den Folgen der Pandemie für die Persönlichkeitsentwicklung von Jugendlichen. Die Erfahrungen des Lockdowns könnten zu Bruchstellen in der Biografie führen, die das gesamte restliche Leben beeinflussen. Sämtliche sozialpsychologischen, soziologischen und pädagogischen Entwicklungsherausforderungen, wie Bildungs- und Qualifikationsprozesse, die Ablösung vom Elternhaus und der Aufbau intensiver Freundschaften sowie Konsum-, Freizeit- und Medienverhalten von Jugendlichen, die in diesem Lebensabschnitt wichtiger werden, sind durch die Einschränkungen in der Pandemie betroffen. Es wird befürchtet, dass Jugendliche mit psychischen, körperlichen, psychosozialen oder psychosomatischen Problemen noch stärker belastet werden und dass Belastungen, wie etwa Kontaktängste oder -störungen, neu und verstärkt auftreten könnten. Zudem halten Jugendforscher eine coronabedingte Zunahme von sozialen und bildungsbezogenen Unterschieden für 
wahrscheinlich, die eine ohnehin schon benachteiligte Gruppe von Jugendlichen noch weiter abhängt (Schmid 2020).

Ähnlich sehen das die Jugendlichen selbst. In einer Studie der Universitäten Frankfurt und Hildesheim zu Jugend und Corona, JuCo, haben 5.128 Jugendliche und junge Erwachsene im Alter zwischen 15 und 30 Jahren teilgenommen und sich zu ihren Erfahrungen in der Pandemie geäußert. Die Jugendlichen und jungen Erwachsenen sehen sich vielfach auf ihre Rolle als Schülerinnen und Schüler reduziert und vermissen, dass auch andere Belange ihrer Altersgruppe diskutiert werden. Dabei sind die meisten zunächst mit der Situation bei sich zuhause überwiegend zufrieden, über 70 \% stimmen der Aussage zu, dass es zuhause immer jemanden gäbe, der sich um sie kümmern würde. Diese Ergebnisse dürfen allerdings nicht darüber hinwegtäuschen, dass es eine kleine Gruppe von Jugendlichen und jungen Erwachsenen von ca. 13 \% gibt, die nur bedingt oder nicht zustimmen, dass sich jemand um sie kümmern würde. Anders sieht es mit der Zufriedenheit bei Kontakten zum Freundeskreis aus. Hier liegt die Zufriedenheit auf einer Skala von 0 (total unzufrieden) bis 10 (zu $100 \%$ zufrieden) im Mittel bei 4,9 und damit wesentlich niedriger als z. B. die Zufriedenheit mit der Stimmung zuhause, die bei einem Durchschnittswert von 6,6 liegt. Auch die Zufriedenheit mit der verbrachten Zeit hat unter Corona sehr gelitten. Während sie vor der Pandemie im Mittel bei 7,4 lag, sind es seit Corona nur noch 5,1. Die Freizeitgestaltung hat sich für $81 \%$ der Befragten angesichts der Einschränkungen (deutlich) verändert. Über ein Drittel der Befragten gibt an, sich in der aktuellen Situation einsam zu fühlen. Es lässt sich festhalten, dass der Wegfall von sozialen Räumen mit den Peers den Jugendalltag grundlegend verändert und den jungen Menschen damit auch alltägliche Bewältigungsmöglichkeiten nimmt, die für den psychosozialen Ausgleich in dieser Lebensphase zentral sind. Wie gravierend die Folgen der Pandemie für junge Menschen sind, zeigt sich auch darin, dass über $45 \%$ angeben, Angst vor der Zukunft zu haben und weitere $23 \%$ zumindest teilweise zustimmen, Angst vor der Zukunft zu haben (Andresen et al. 2020a).

Zentral ist auch die zeitliche Dimension: Der erste Lockdown war zunächst eine einmalige Ausnahme, die Kräfte und Verständnis für die notwendigen Maßnahmen freigesetzt hat, der zweite Lockdown kann dadurch als belastender empfunden werden. Auch unterscheidet sich das Zeitempfinden von Kindern und Erwachsenen, mehrere Wochen sind für Kinder unendlich lange, zumal, wenn es keinen festen Endpunkt zu normalem Schulunterricht bzw. Kitabesuch gibt. 


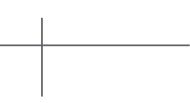

\subsection{Weitere Entwicklung, kinderbewusste Pandemie- und Schulpolitik sowie Therapieangebote}

Die Pandemie, die Veränderungen der Alltagsstrukturen und die Folgen werden von vielen als sehr belastend gesehen. Empirisch nachweisbar ist eine Verstärkung von psychischer Belastung durch die Corona-Pandemie bei Erwachsenen (z. B. Knolle et al. 2021), Kindern (Langmeyer et al. 2020; Ravens-Sieberer et al. 2021) und Jugendlichen, wie die hier gezeigten Analysen der pairfam Corona-Auswertung deutlich zeigen (Abb. 7). All diesen Ergebnissen liegen Daten aus dem Frühjahr 2020 zugrunde. Durch die Dauer der Pandemie und die wiederholten Einschränkungen, Schulschließungen und Einschränkungen sozialer Kontakte kann davon ausgegangen werden, dass sich die Situation im Pandemieverlauf noch verstärkt hat.

Ob sich die psychologischen Belastungen jedoch als klinisch relevante Diagnosen manifestieren oder wie dies abgewendet werden kann, sind essentielle Fragen. Wie kann es gelingen, dass von den psychisch belasteten Jugendlichen möglichst viele psychisch gesund aus der Pandemie kommen? Vier Punkte sind hier zu nennen:

- Ein zentraler Faktor ist, dass bei einer möglichen vierten Welle die Öffnung von Schulen prioritär ist. Bei zukünftigen Entscheidungen zur Bekämpfung der Pandemie sollte das Wissen zu Auswirkungen von Schulschließungen auf Bildung und Gesundheit von Kindern und Jugendlichen ebenso einfließen wie es zu Inzidenzen, der Krankenhaussituation, Arbeitsmarkt und zu Reisen der Fall ist.

- Die Schulen sollten beim Nachholen des Lernstoffes nicht unnötig Druck auf die Schülerinnen und Schüler aufbauen und dafür ein realistisches Maß an Zeit - eher Jahre statt Monate - einplanen. Auch sollte klar kommuniziert werden, dass Lernrückstände nicht Defizite oder gar „Schuld“ der Schülerinnen und Schüler sind, sondern Folgen der pandemiebedingten Schulschließungen. Wie ordnet ein Kind es ein, wenn es deutlich schlechtere Noten als vor der Pandemie bekommt? Wissen über die Bedeutung von internaler und externaler Attribution für das Selbstbewusstsein kann helfen, die Kommunikation in Medien, Politik und den Schulklassen so zu gestalten, dass Kinder und Jugendliche sich für die schulischen Defizite nicht auch noch selbst die Schuld geben.

- Schule sollte nicht nur als Ort der Wissensvermittlung und der Distanzunterricht nicht nur als Gefahr für Bildungsdefizite verstanden werden. Vielmehr muss Schule als sozialer Lernraum betrachtet werden. Das Zusammenkommen Gleichaltriger, 
Auseinandersetzungen und ein Miteinander muss nach der Schulschließung und dem Wechselunterricht erst wieder aufgebaut werden.

- Für belastete Kinder und Jugendliche sollten Beratungs- und Unterstützungsangebote und, wo nötig, auch Therapieplätze zur Verfügung stehen. Für die psychische Gesundheit der Kinder und Jugendlichen sollten gerade jetzt im Zuge der Mehrbelastung durch die Corona-Pandemie unterschiedliche Bereiche der psychischen Hilfen und Versorgung gestärkt werden. Zum einen der ambulante Sektor, der unter anderem niedergelassene Therapeutinnen und Therapeuten und Erziehungs- und Familienberatungsstellen umfasst. Zum anderen der stationäre Sektor, der Kliniken für Kinder- und Jugendpsychiatrie, aber auch Institutionen der Kinder- und Jugendhilfe beinhaltet. Als dritter Baustein der schulische Bereich, mit Schulpsychologinnen und -psychologen, Schulsozialarbeiterinnen und -arbeitern und Schulen für Erziehungshilfe.

Die Therapieanfragen für Kinder und Jugendliche sind während der Corona-Pandemie deutlich gestiegen (Deutsches Ärzteblatt 2021). Die zusätzlichen Anfragen treffen auf ein System, welches bereits vor der Pandemie viele Kinder und Jugendliche mit Hinweisen auf psychische Auffälligkeiten nicht ausreichend berücksichtigt hat. So berichtet die BELLA-Studie, dass nur $22 \%$ der entsprechenden Kinder und Jugendlichen innerhalb eines Jahres Kontakt mit Fachversorgern hatten (Klasen et al. 2017). Die Situation seit März 2020 legt nun offen, was schon länger bekannt ist: Regionale Versorgungsdefizite, die sich insbesondere bei gestiegenem Beratungsbedarf durch längere Wartezeiten auf einen Therapieplatz zeigen (Deutsche Gesellschaft für Kinder- und Jugendpsychiatrie, Psychosomatik und Psychotherapie 2021). Zum Tragen kommt hierbei zusätzlich, dass soziale Ungleichheiten damit verstärkt werden. Während manche Familien in der Lage sind, Therapie auch von privaten Psychotherapeutinnen und -therapeuten zu bezahlen, warten andere lange auf einen Platz bei einer niedergelassenen Therapeutin bzw. einem Therapeuten mit Kassenzulassung. Dies zeigte sich bereits vor der Pandemie und verstärkt sich durch die gegenwärtige Lage.

Im Zuge der Corona-Pandemie haben viele Einrichtungen der Familienbildung und Familienberatung neue Angebote, insbesondere digitale Formate, geschaffen. Beratungsstellen haben relativ schnell wieder ihre Arbeit aufgenommen und waren weniger von Schließungen betroffen als Familienbildungsstätten. Jedoch gab es bei den Beratungsstellen regionale Unterschiede und Unterschiede hinsichtlich der Durchführung und des Formats von Beratungsangeboten. Die digitalen Formate konnten zwar implementiert werden, erreichten allerdings nicht alle Familien. Vor allem Familien mit wenig Internetkenntnissen und Familien mit Migrationshintergrund blieben häufig außen vor (Juncke et al. 2021). 
Wenn nun die Schulen wieder im regulären Präsenzunterricht geöffnet haben, Vereine und Bildungseinrichtungen wieder öffnen, wird vor allem durch Lehrkräfte, (Sozial-) Pädagoginnen und -pädagogen oder Erzieherinnen und Erzieher der Hilfebedarf in vielen Familien und bei vielen Jugendlichen deutlich werden. Er wird vor allem bei Jugendlichen und Kindern in Erscheinung treten, die bereits vor der Pandemie gefährdet oder beeinträchtigt waren. Oftmals sind es die genannten Vermittlerinnen und Vermittler, denen psychische Probleme bei Kindern und Jugendlichen auffallen und die Hilfen initiieren. Es kann davon ausgegangen werden, dass sämtliche Angebote zur psychischen Gesundheit noch deutlich stärker gefordert werden, wenn die Kinder und Jugendlichen wieder in Institutionen und Freizeitaktivitäten eingebunden werden (Fegert et al. 2021), und dass die Hilfeanfragen die Angebote dann klar übersteigen.

So fordert die Bundespsychotherapeutenkammer eine generelle deutliche Ausweitung des Angebots der Psychotherapie (Bühring 2021) und die International Union for Health Promotion and Education (2021) appelliert, die Pandemie zu nutzen, um die psychische Gesundheit stärker in den Fokus zu nehmen, breite Angebote zu schaffen, Investitionen in Personal und Organisationen zu tätigen und Wirksamkeiten von Strategien zu überprüfen.

Es wird viele unterschiedliche Anstrengungen benötigen, denn „Schulöffnungen alleine werden leider kinder- und jugendpsychiatrische Störungen nicht beheben“ (Deutsche Gesellschaft für Kinder- und Jugendpsychiatrie, Psychosomatik und Psychotherapie 2021). Trotzdem sollte nicht, wie häufiger aus politischen Kreisen und in den Medien zu hören ist ${ }^{4}$, von einer „verlorenen Generation“ gesprochen werden. Die meisten Kinder und Jugendlichen haben bei aller Schwierigkeit Wege gefunden, die Phase der Schulschließungen gesund zu überstehen und anschließend Schritt für Schritt die gewohnte Lebensweise wieder zu erlangen. Manche werden dabei Resilienz entwickeln und das Selbstbewusstsein, Krisen meistern zu können. Es geht vielmehr darum, die belasteten Kinder und Jugendlichen auf allen Ebenen zu erreichen, zu stärken und ggf. eine Pathologisierung zu verhindern. Vor allem sollten Politik und Gesellschaft den hohen Preis von Schulschließungen für die Kinder und Jugendlichen richtig einschätzen und bei der zukünftigen Pandemiepolitik berücksichtigen. 


\section{Belastungen von Eltern im Lockdown}

Betrachtet man Kinder und Jugendliche in der Pandemie, kommt man nicht umhin, auch die Eltern zu berücksichtigen, da Kinder und Jugendliche sowie ihre Eltern Teile des Systems Familie sind. Belastungen innerhalb der Familie haben häufig Auswirkungen auf alle Mitglieder. Hier gilt die These: Zufriedene Eltern sind gute Eltern.

Eltern sind durch die Corona-Krise in mehrfacher Hinsicht betroffen. Erstens erfuhren sie die gleichen Veränderungen am Arbeitsmarkt wie viele andere Beschäftigte: Die einen wechselten ins Homeoffice, die anderen waren in systemrelevanten Berufen besonders stark am Arbeitsplatz gefordert, und wieder andere hatten durch Kurzarbeit, Freistellung, Arbeitslosigkeit oder den Wegfall von Aufträgen als Selbständige weniger oder gar keine Arbeit. Zweitens waren bzw. sind Eltern durch die wiederholte Schließung von Schulen und Kitas sowie den Ausfall der Großeltern als Betreuungspersonen phasenweise alleinverantwortlich für die Betreuung und Beschulung ihrer Kinder. Drittens sorgen Eltern sich um ihre Kinder, z. B. mit Blick auf deren psychische und physische Gesundheit, den Bildungserfolg und die soziale altersgerechte Entwicklung.

Wie sich diese Gemengelage auf das Belastungsempfinden von Eltern auswirkt, ist Gegenstand dieses Kapitels. Im ersten Abschnitt (6.1) wird ein Überblick zur Entwicklung der Belastung von Eltern über die Zeit gegeben. Dabei stellen wir zunächst den aktuellen Forschungsstand bisheriger Studien vor und präsentieren anschließend neue Analysen mit Daten des deutschen Familienpanels pairfam zur subjektiv wahrgenommenen Aktivität, zum Stressempfinden und zur Einsamkeit. Im zweiten Abschnitt (6.2) werden auf Basis eines Literaturüberblicks Veränderungen der geschlechtsspezifischen Arbeitsteilung in Familien nachvollzogen. Anschließend (Abschnitt 6.3) wird die Entwicklung der Belastungen speziell mit Blick auf kinderbezogene Aspekte, insbesondere Kinderbetreuung und Homeschooling, dargestellt. Der folgende Abschnitt 6.4 stellt die 
Folgen hoher Belastung, wie z. B. Niedergeschlagenheit und Erschöpfung, dar. Darauf folgt im abschließenden Abschnitt 6.5 die Betrachtung der Risikofaktoren für eine hohe elterliche Belastung, wie z. B. Geschlecht, Partnerschaftsstatus und Erwerbssituation. Vorangeschickt werden sollte noch, dass die aktuellen Forschungserkenntnisse teilweise aus Studien stammen, deren Stichproben nicht repräsentativ sind, da es sich um Convenience Samples oder Schneeballsamples handelt ${ }^{5}$. Zudem sind einige Studien nur im Querschnitt angelegt, was keinen Vergleich mit der Situation vor der Pandemie oder zu verschiedenen Zeitpunkten während der Pandemie ermöglicht.

\subsection{Belastungen von Eltern im Zeitverlauf}

\section{BISHERIGE STUDIEN}

Die Böckler-Erwerbstätigenbefragung (Hövermann 2021) zeigt anhand von vier Messzeitpunkten (April, Juni, November 2020 sowie Januar 2021) auf, wie stark sich Eltern im Verlauf der Pandemie durch die Gesamtsituation belastet fühlten. Wie Abbildung 13 auf Basis dieser Studie zeigt, waren Eltern mit Kindern unter 14 Jahren bereits im ersten Lockdown deutlich belasteter als andere Erwerbspersonen. So gaben $45 \%$ der Väter, aber nur 38 \% aller befragten Männer an, durch die Gesamtsituation „äußerst“ oder „stark“ belastet zu sein. Bei den Müttern waren es sogar 52 \%, verglichen mit $46 \%$ aller Frauen.

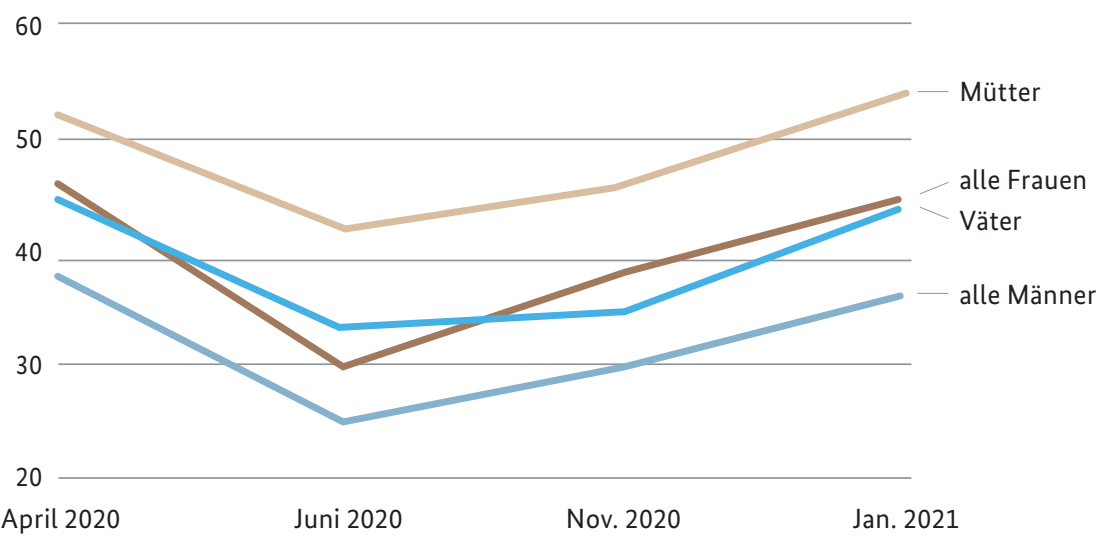

Abbildung 13: Entwicklung der Belastung durch die Gesamtsituation zwischen April 2020 und Januar 2021 (Angaben in \%)

Quelle: Eigene Darstellung auf Basis von Hövermann (2021). 
Bis zum Juni 2020 sank die Belastung in allen Gruppen ab. Am deutlichsten war hier der Rückgang bei den Frauen insgesamt (16 Prozentpunkte); am schwächsten fiel er bei den Müttern aus (10 Prozentpunkte) - hier lag der Anteil belasteter Mütter auch im Juni noch bei $42 \%$. Bis zum November stiegen die Belastungen dann in allen vier Gruppen wieder an, und wiederum waren Mütter und Väter häufiger belastet als jeweils Frauen und Männer insgesamt. Die Belastungen erreichen im November allerdings (noch) nicht wieder das Niveau des ersten Lockdowns. Dies liegt vermutlich daran, dass sich Deutschland zu dem Zeitpunkt noch in einem Teillockdown befand, in dem zwar Bars, Restaurants etc. geschlossen hatten, aber Kitas, Schulen und Geschäfte noch geöffnet waren. In der zweiten Lockdown-Phase ab Dezember, in der auch Kitas und Schulen geschlossen waren, stieg die Gesamtbelastung unter Eltern jedoch weiter. Zum letzten Messzeitpunkt im Januar 2021 berichteten 54 \% der Mütter von einer hohen Gesamtbelastung, was den höchsten Wert seit Beginn der Befragung darstellt. Für Väter lag der Anteil mit hoher Belastung mit 44 \% auf einem ähnlichen Niveau wie im ersten Lockdown im April 2020.

In der österreichischen SORA-Studie (Ringler und Baumegger 2020) wird die Belastung von Eltern mit Kindern bis 14 Jahren während des ersten und zweiten Lockdowns (April und November 2020) verglichen. Es zeigt sich ein Anstieg der Belastungen zwischen diesen beiden Zeitpunkten: Unter den Vätern stieg der Anteil der Belasteten von $40 \%$ auf $51 \%$ und bei den Müttern von $51 \%$ auf $64 \%$. Die Autoren begründen dies damit, dass der Arbeitsdruck und die Erwartungen der Arbeitgeberinnen und -geber im zweiten Lockdown höher waren als im ersten, wohingegen die Bildungseinrichtungen im November zumindest für die Betreuung offen hatten. Als weiterer möglicher Grund wird angeführt, dass die Eltern sich über den Sommer nicht ausreichend erholen konnten, da viele Urlaubstage im ersten Lockdown bereits für die Kinderbetreuung aufgewendet werden mussten.

Auch die im August 2020 durchgeführte Studie von Calvano et al. (2021), in der die Eltern retrospektiv nach ihrem Stresslevel in den vergangenen Monaten gefragt wurden, weist auf einen signifikanten Anstieg im Niveau „elternschaftsbezogenen Stresses“ in der Pandemie hin. Der „allgemeine Stress“ hingegen lag der Studie zufolge auch während der Pandemie auf niedrigem Niveau. Zudem bestätigt die internationale COVIDiSTRESS-Studie mittels multivariater Analysen auf Basis einer Stichprobe von Befragten aus 26 Ländern, dass Eltern im März und April 2020 signifikant stärker gestresst waren als kinderlose Personen (Kowal et al. 2020).

Darüber hinaus existieren Erkenntnisse zum Thema Belastungen und Stress aus reinen Elternbefragungen, die keinen Vergleich über die Zeit oder mit Kinderlosen bieten und 
deren Ergebnisse daher schwieriger einzuordnen sind. So berichten in der Forsa-Elternumfrage, die im Auftrag der DAK während des ersten Lockdowns durchgeführt wurde, $48 \%$ der Eltern von Stress während der Schulschließungen - $32 \%$ waren oft und $16 \%$ sogar sehr oft gestresst. Mütter waren dabei mit $49 \%$ häufiger gestresst als Väter mit $45 \%$ (Forsa 2020a). Auch in der Studie „Familien \& Kitas in der Corona-Zeit“ der Universität Bamberg berichteten viele Eltern von Kindern zwischen 0 und 6 Jahren im April/Mai 2020, sich durch die Vielzahl an Belastungen gestresst zu fühlen - im Durchschnitt 3,1 von 4 Punkten (Cohen et al. 2020). Demgegenüber zeigt eine Studie des Instituts für Demoskopie Allensbach, dass ein Großteil der Eltern mit Kindern unter 15 Jahren (59 \%) im April/ Mai der Meinung war, bisher ganz gut durch die Krise gekommen zu sein (BMFSFJ 2020).

\section{EIGENE ANALYSEN}

Wie auch in Kapitel 5.1 werden nachfolgend eigene Analysen mit dem Familienpanel pairfam dargestellt. An der pairfam-Covid-19-Zusatzstudie nahmen zwischen dem 19. Mai und dem 13. Juli 20201.938 pairfam-Befragte im Alter zwischen 24 und 48 Jahren teil. Ihnen wurden dieselben Fragen gestellt wie in den Jahren zuvor, etwa danach, wie gestresst, überlastet, tatkräftig oder allein sie sich in den letzten vier Wochen überwiegend gefühlt haben. Die Antworten auf die Fragen wurden anhand einer 5-er Skala von 1 (trifft überhaupt nicht zu) bis 5 (trifft voll und ganz zu) erhoben. Somit lässt sich die Lebenssituation der Befragten vor und während der Corona-Krise, insbesondere gegen Ende des ersten Lockdowns, vergleichen.

Unsere Analysen zeigen, dass im Vergleich zu der Zeit vor der Pandemie ein statistisch signifikanter Rückgang der subjektiv wahrgenommenen Aktivität bei den Erwachsenen im Alter von 24 bis 48 Jahren konstatiert werden kann (Abb. 14). Das heißt, wie bereits bei den Jugendlichen deutlich wurde (Kap. 5.1.), fühlten sich auch die Erwachsenen im Frühjahr 2020 deutlich weniger tatkräftig, aktiv und waren weniger energiegeladen als noch im Vorjahr.

Jedoch hat sich die Gefühlslage der Bevölkerung in Deutschland durch die Corona-Pandemie und die damit verbundenen Einschränkungen ganz unterschiedlich verändert. Denn zugleich waren die Befragten weniger gestresst, überlastet und hatten auch ein geringeres Gefühl, unter Druck zu stehen. Die Unterschiede zu der Zeit vor der Pandemie sind in diesem Fall ebenfalls statistisch signifikant. Die Entschleunigung des alltäglichen Lebens durch die Verringerung sozialer Kontakte, die Einschränkung von Freizeitaktivitäten und das Arbeiten im Homeoffice scheinen also unter anderem mit einem geringeren Stressempfinden einherzugehen (Abb. 15). Im Vergleich dazu fallen die stressauslösenden 


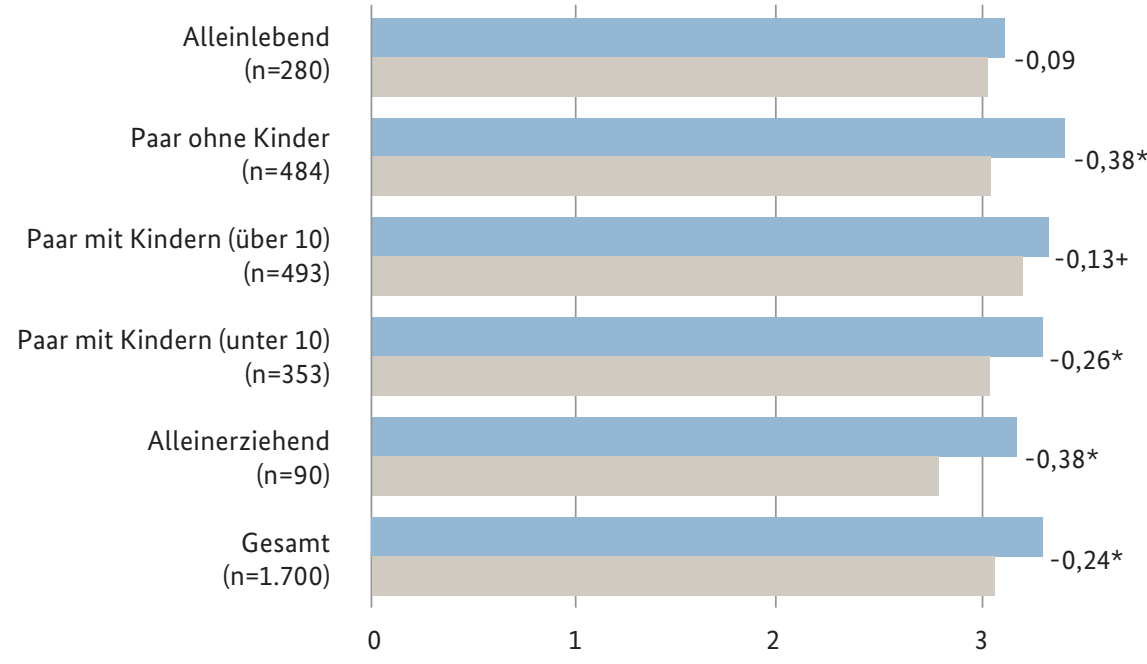

Vor COVID-19-Pandemie (2018-19)

Während COVID-19-Pandemie (Mai/Juni2020)

\section{Abbildung 14: Veränderung von subjektiv wahrgenommener Aktivität nach Familiensituation}

* $p<0,05 ;+p>0,1 ; n=$ Fallzahl; eigene Analysen. Datenquelle: pairfam Release 11.0 und pairfam Covid-19-Survey. Anmerkung: Wahrgenommene Aktivität setzt sich aus drei Komponenten „aktiv“, „tatkräftig“ und „voller Energie"zusammen.

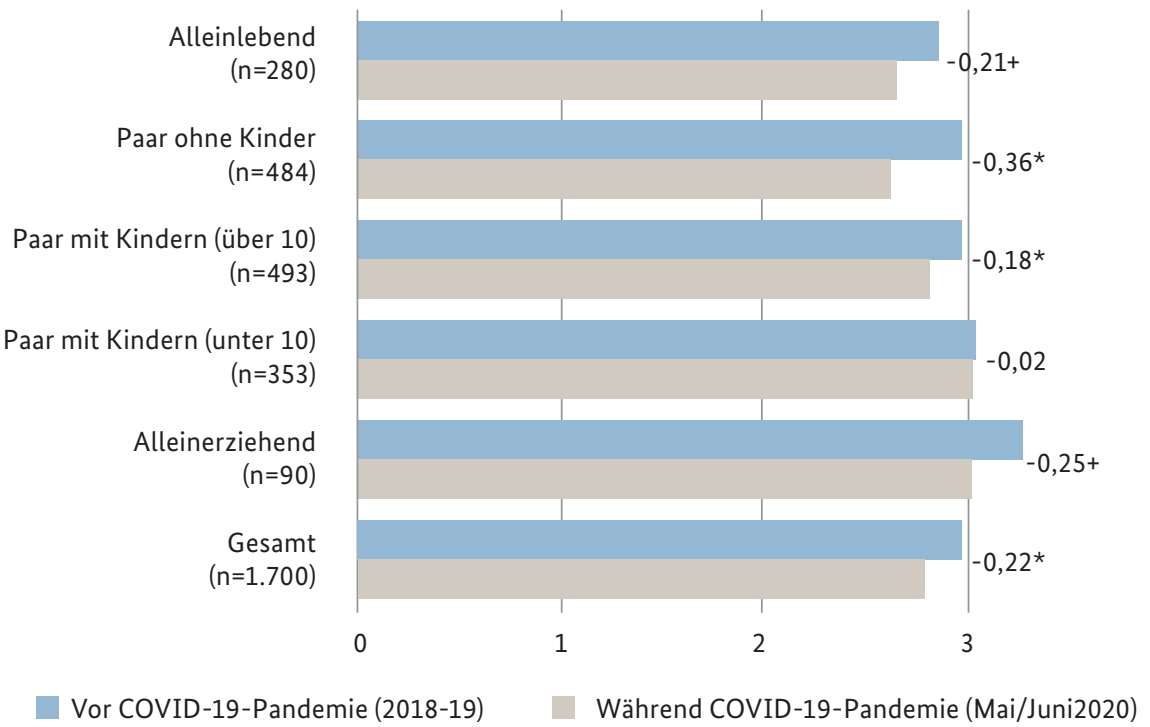

\section{Abbildung 15: Veränderung von Stressempfinden nach Familiensituation}

${ }^{*} p<0,05 ;+p>0,1 ; n=$ Fallzahl; eigene Analysen. Datenquelle: pairfam Release 11.0 und pairfam Covid-19-Survey. 
Ängste, Unsicherheiten bezüglich der eigenen beruflichen Zukunft oder die Herausforderung, Kinderbetreuung und Homeschooling mit Homeoffice zu vereinbaren, offenbar weniger stark aus. Zumindest für die Zeit am Ende des ersten Lockdowns kann festgehalten werden, dass nicht nur negative Aspekte, sondern auch positive Empfindungen von den Befragten wahrgenommen werden (siehe Schmiedeberg und Thönnissen 2021).

Hinsichtlich der wahrgenommenen Einsamkeit scheint sich auf den ersten Blick kein Zusammenhang mit den Kontaktbeschränkungen zu zeigen. Wie die Abbildung 16 deutlich macht, steigt dieser Wert im Vergleich zu der Zeit vor der Pandemie nur marginal.

Unsere Ergebnisse zeigen, dass Alleinlebende verhältnismäßig gut durch den ersten Lockdown gekommen sind. Ihr Stressempfinden geht leicht zurück, sie sind vergleichsweise wenig von einer Verringerung ihrer Aktivität betroffen und auch ihr Einsamkeitsgefühl hat nicht zugenommen. Zu beachten ist, dass wir keine Aussagen über ältere Alleinlebende über 50 Jahre machen können, da diese Altersgruppe nicht an der Studie teilgenommen hat. Andere Studien konnten aber bereits zeigen, dass das Einsamkeitsrisiko besonders bei Alleinlebenden im mittleren und hohen Erwachsenenalter ansteigt (Huxhold und Tesch-Römer 2021).

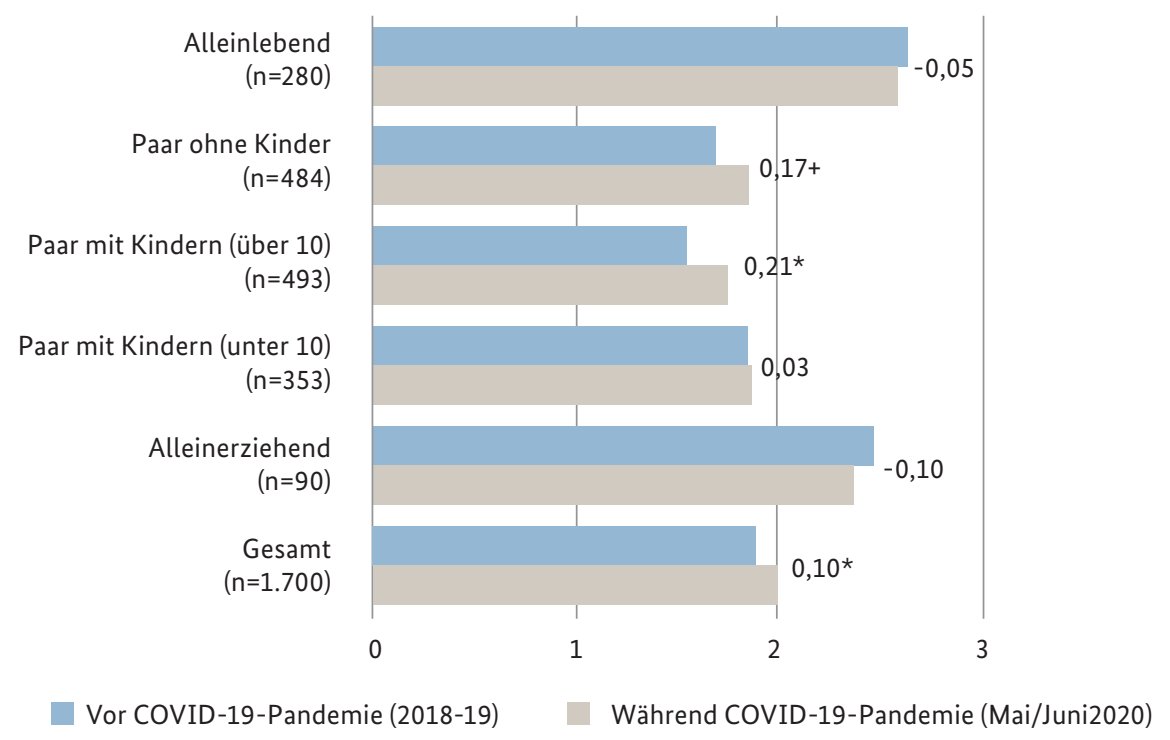

Abbildung 16: Veränderung von subjektiv wahrgenommener Einsamkeit nach Familiensituation ${ }^{*} p<0,05 ;+p>0,1 ; n=$ Fallzahl; eigene Analysen. Datenquelle: pairfam Release 11.0 und pairfam Covid-19-Survey. 
Die Aktivität von kinderlosen Paaren geht im Vergleich zu allen anderen Bevölkerungsgruppen - mit Ausnahme der Jugendlichen - besonders stark zurück. Bei der Belastung von Familien mit Kindern spielt das Alter der Kinder eine wichtige Rolle. Nur Eltern von jüngeren Kindern unter 10 Jahren, die entsprechend deutlich mehr Betreuung und Unterstützung brauchen, erleben keine nennenswerte Verringerung des Stressempfindens.

Insgesamt zeigt sich also kurzfristig ein gemischtes Bild mit positiven und negativen Veränderungen des Wohlbefindens der Bevölkerung zum Ende des ersten Lockdowns. Während weniger Stress zuträglich für die Gesundheit ist, sind die Zunahme von Einsamkeit und die Verringerung der subjektiv wahrgenommenen Aktivität Risikofaktoren für das Wohlbefinden und die psychische Gesundheit.

Die Einschränkungen in den einzelnen Lebensbereichen scheinen auf die Menschen in Deutschland ganz unterschiedliche Auswirkungen zu haben. Mitunter ist auch die jeweilige Familiensituation dabei entscheidend, welche Auswirkungen als besonders belastend erlebt werden, aber auch welche Möglichkeiten der Person zur Verfügung stehen, mit den Herausforderungen umzugehen. Damit die Politik gezielt unterstützen kann, ist es wichtig, besonders belastete Risikogruppen zu identifizieren.

Allerdings muss bei der Interpretation der Ergebnisse beachtet werden, dass auch weitere Faktoren auf die jeweiligen hier untersuchten Personengruppen einen Einfluss haben können. Unsere Daten zeigen, dass es zwar kaum Unterschiede in der Inanspruchnahme von Homeoffice gibt (der Anteil derjenigen, die angeben, dass sie im Homeoffice gearbeitet haben, liegt in allen Gruppen zwischen 34 und $40 \%$ ), allerdings sind Paare mit Kindern unter 10 Jahren deutlich häufiger von Kurzarbeit (26\%) oder Jobverlust ( $8 \%$ ) betroffen. Bei den Alleinlebenden, den Paaren ohne Kinder oder den Paaren mit älteren Kindern liegt der Anteil der Kurzarbeiter zwischen $12 \%$ und $18 \%$ bzw. beim Jobverlust zwischen $3 \%$ und $5 \%$.

\subsection{Zeitliche Mehrbelastung: Verteilung zwischen den Geschlechtern}

Zu Beginn der Pandemie gab es in der Öffentlichkeit Befürchtungen, wonach der erste Lockdown eine Retraditionalisierung der Geschlechter bei Eltern bewirkt hat. In der öffentlichen Debatte scheint es leichter zu sein, „Mütter als Opfer darzustellen, als ein differenziertes Bild“, das auch engagierte Väter kennt, zu zeichnen (Kreyenfeld und Bujard 2021). Beobachtungen hinsichtlich einer Retraditionalisierung gab es dahingehend, 
dass über 20 \% der Mütter ihre Arbeitszeit zu Beginn des ersten Lockdowns reduziert haben, und dass Forscher, aber nicht Forscherinnen, zu Beginn der Pandemie besonders produktiv waren (Allmendinger 2020). Im Laufe der Pandemie gab es eine Vielzahl von Befragungen, wobei repräsentative Panelstudien wie die Mannheimer Corona-Studie (Befragung April 2020, Möhring et al. 2020), die pairfam-Covid-Zusatzstudie (Mai bis Juli 2020) und die SOEP-CoV-Studie, ein Sub-Sample des Sozio-oekonomischen Panels (April bis Juni 2020), für die jeweiligen Befragungszeiträume wertvolle Erkenntnisse geliefert haben, auch da sie als Wiederholungsbefragungen einen Vergleich zur Vor-Corona-Zeit ermöglichen.

So zeigen Analysen des BiB anhand der Mannheimer Corona-Studie, dass in der ersten Lockdownphase Ende April 2020 Mütter zwar deutlich mehr Familienarbeit als Väter geleistet haben, dass der Anteil der Väter an der Familienarbeit im Durchschnitt aber sogar etwas angestiegen ist, von 33 \% (im Jahr 2018) auf 41 \% (Bujard et al. 2020b). Damit sprechen die Ergebnisse des BiB gegen die These der Retraditionalisierung für den ersten Lockdown.

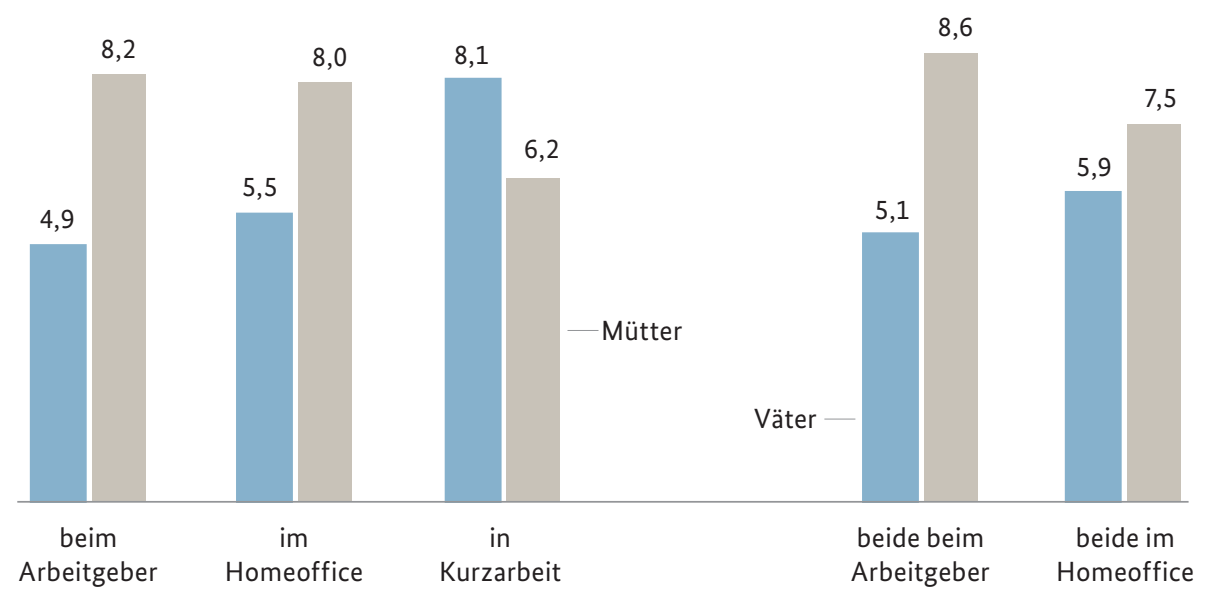

Abbildung 17: Zeit für Familienarbeit im April 2020 (durchschnittliche Stundenanzahl an einem normalen Werktag)

Quelle: Eigene Darstellung, Datenquelle: Mannheimer Corona-Studie 2020.

Anmerkungen: Auswertungen beziehen sich auf Eltern, die mit Kindern unter 16 Jahren im Haushalt leben. Es wird nicht die Zeitverwendung von Paaren betrachtet, da keine Partnerbefragung vorliegt, sondern Individuen sortiert nach eigenem Arbeitsort und dem des Partners. Familienarbeit beinhaltet Fürsorgearbeit und Hausarbeit. Kurzarbeit beinhaltet auch zu geringen Anteilen „freigestellt mit oder ohne Lohn“. 
Hinter diesen Durchschnittswerten verbirgt sich eine große Heterogenität: Der Anstieg bei den Vätern ist unter anderem auf die hohe Zahl von Vätern in Kurzarbeit zurückzuführen, die in dieser Phase im Durchschnitt acht Stunden Familienarbeit geleistet haben (Bujard et al. 2020b). Väter, die beim Arbeitgeber tätig waren, haben werktags 4,9 Stunden Familienarbeit geleistet. Väter, welche im Homeoffice gearbeitet haben, haben etwa 5,5 Stunden in die Familienarbeit investiert (Abb. 17). Mütter haben sowohl im Homeoffice als auch bei einer Arbeit beim Arbeitgeber etwa acht Stunden werktags Familienarbeit geleistet. Dabei ist zu bedenken, dass viele von ihnen nur in Teilzeit erwerbstätig waren.

Interessant ist der Blick auf Väter und Mütter, die den gleichen Arbeitsort wie ihre Partnerin bzw. ihr Partner hatten: Bei Eltern, die beide am Arbeitsplatz gearbeitet haben, haben Mütter erheblich mehr Familienarbeit geleistet als Väter (8,6 zu 5,1 Stunden, Abb. 17). Wenn beide im Homeoffice gearbeitet haben, wurde die Familienarbeit etwas egalitärer verteilt (7,2 zu 5,9 Stunden). Dies lässt sich vermutlich dadurch erklären, dass Väter im Homeoffice den Bedarf an Betreuung und Hausarbeit in ihrem Alltag gesehen haben, und durch den Wegfall der Pendelstrecken auch mehr Zeit für Familienarbeit hatten.

Die Mannheimer Corona-Studie hat auch gezeigt, wie sehr der Lockdown zur Betreuung der Kinder durch die eigenen - meist erwerbstätigen - Eltern geführt hat: Zwischen dem 20. März und 2. April 2020 wurden Kinder unter 16 Jahren werktags zu $92 \%$ von Personen aus dem eigenen Haushalt (meistens Eltern) betreut, nur $1 \%$ der Kinder war in Notbetreuung, der Rest wurde nicht oder durch Personen aus einem anderen Haushalt betreut (Möhring et al. 2020).

Daten der SOEP-CoV-Befragung für den Befragungszeitraum 30. März bis 30. Mai 2020 bestätigen die Ergebnisse der Auswertungen der BiB-Studie, da sie ebenfalls einen stärkeren Anstieg der Familienarbeit bei Vätern zeigen (Tab. 3). Auch zeigen sie, dass die Heterogenität der Arbeitszeit für Kinderbetreuung deutlich zugenommen hat. Bemerkenswert ist der Befund, dass es erhebliche Bildungsunterschiede bei den Veränderungen der geschlechtsspezifischen Arbeitsteilung gab (Kreyenfeld und Zinn 2021): Der Anstieg der Familienarbeit bei den Vätern zeigt sich kaum bei den Akademikern, die normalerweise egalitärere Einstellungen vertreten und vor der Corona-Pandemie mit 3,6 Stunden pro Tag mehr Familienarbeit leisteten als die mittlere Bildungsgruppe der Väter mit 2,3 Stunden. Bei Vätern mit höherem Bildungsniveau ist die Kinderbetreuungszeit nur geringfügig um 36 Minuten in der Pandemie angestiegen, bei den Vätern mit mittlerem Bildungsniveau hat sie sich mehr als verdoppelt auf insgesamt 6 Stunden pro Tag (Abb. 18). 


\begin{tabular}{|c|c|c|c|c|c|c|}
\hline & \multirow{2}{*}{$\begin{array}{l}\text { Befragungs- } \\
\text { zeitraum }\end{array}$} & \multicolumn{3}{|c|}{ Familienarbeit (in Stunden pro Tag) } & \multicolumn{2}{|c|}{ Anteil Familienarbeit (in \% } \\
\hline & & Mutter & Vater & Summe & Mutter & Vater \\
\hline \multirow{3}{*}{$\begin{array}{l}\text { Mannheimer } \\
\text { Corona-Studie }\end{array}$} & 2018 & 6,6 & 3,3 & 9,9 & 67 & 33 \\
\hline & $4 / 2020$ & 7,9 & 5,6 & 13,5 & 59 & 41 \\
\hline & Anstieg (in \%) & 20 & 70 & 36 & & \\
\hline \multirow[t]{3}{*}{ SOEP-COV } & 2019 & 6,7 & 2,8 & 9,5 & 71 & 29 \\
\hline & $4-5 / 2020$ & 9,6 & 5,3 & 14,9 & 64 & 36 \\
\hline & Anstieg (in \%) & 43 & 89 & 57 & & \\
\hline
\end{tabular}

\section{Tabelle 3: Veränderung der Arbeitsteilung im ersten Lockdown}

Quelle: Kreyenfeld und Zinn 2021, S. 108; Bujard et al. 2020b, S. 37.

Anmerkungen: Unterschiede im Studiendesign: Bujard et al. (MCS) beziehen sich auf Personen, die vor der Krise erwerbstätig waren, Kreyenfeld und Zinn (SOEP) haben auch nichterwerbstätige Eltern im Sample. Kreyenfeld und Zinn analysieren nur die Zeit für Kinderbetreuung, Bujard et al. berücksichtigen die Zeit für Kinderbetreuung und Hausarbeit.

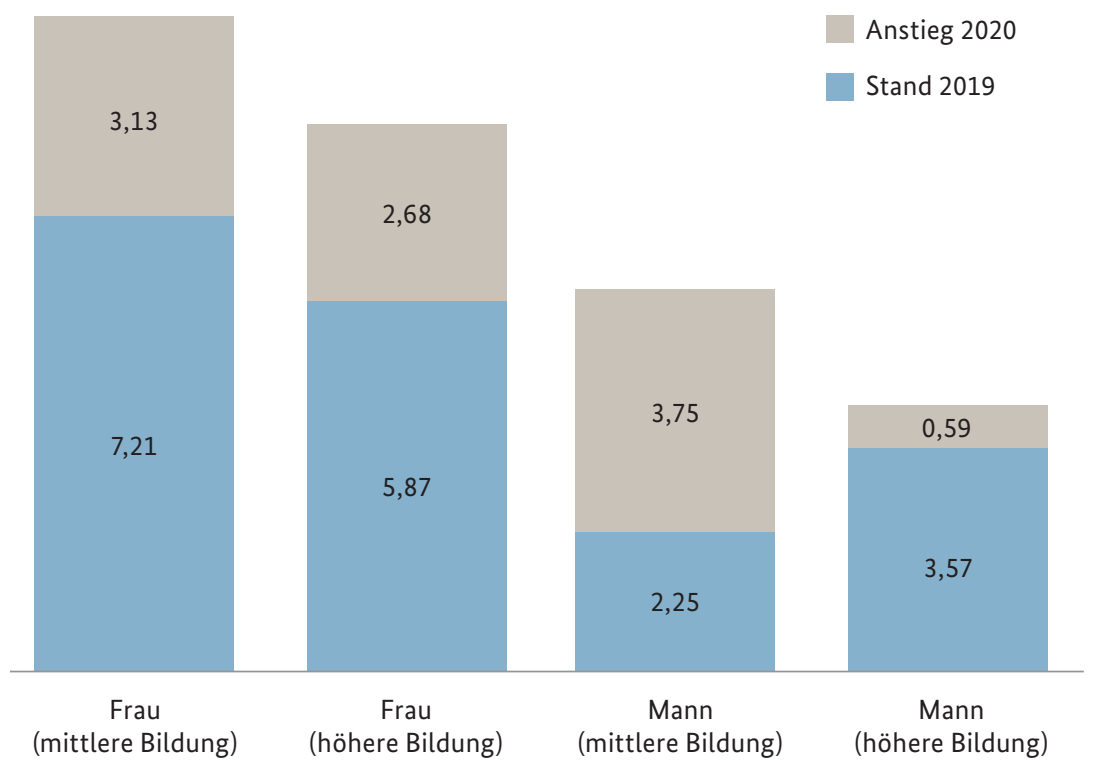

\section{Abbildung 18: Zeit für Kinderbetreuung: Anstieg im Lockdown gegenüber 2019 (in Stunden pro Tag, Alter des jüngsten Kindes unter 12 Jahren)}

Quelle: Eigene Darstellung basierend auf: Kreyenfeld und Zinn 2021. 
Diese Bildungsunterschiede könnten mit dem von der BiB-Studie gezeigten hohen Anteil der Familienarbeit bei Vätern in Kurzarbeit zusammenhängen. Differenziert man nach Alter der Kinder, zeigt sich der größte Anstieg bei Eltern von Kindern im Alter von 3 bis 5 Jahren mit zusätzlich 3,5 Stunden für Väter und 4 Stunden für Mütter (Kreyenfeld und Zinn 2021). Dies liegt vermutlich daran, dass das Ausgangsniveau für Fürsorgearbeit bei Kindern unter drei Jahren bereits vor der Corona-Pandemie sehr hoch war. Kinder im Alter zwischen 3 und 5 Jahren besuchten vor der Krise deutlich häufiger als jüngere Kinder eine Kita, so dass deren Eltern daher auch häufiger durch die Kitaschließungen direkt betroffen waren.

Andere Studien beobachten ebenfalls ein sehr heterogenes Geschehen bezüglich der geschlechtsspezifischen Arbeitsteilung. Für die Phase des auslaufenden Lockdowns Mai bis Juli 2020 lassen sich mit pairfam-Daten keine klaren Trends hinsichtlich der Arbeitsaufteilung der Paare feststellen (Hank und Steinbach 2021). Auch diese Studie konnte zeigen, dass Homeoffice zu einer höheren Beteiligung an der Familienarbeit im Vergleich zu Vor-Pandemie-Zeiten beiträgt, und dass der Anstieg der Hausarbeit stärker bei den Müttern und der Anstieg der Kinderbetreuung stärker bei den Vätern war. Eine Studie auf Basis von Daten der Zusatzbefragung des Nationalen Bildungspanels (NEPS) (Zoch et al. 2020a; 2020b) gibt Aufschluss über die Arbeitsteilung innerhalb von Familien im Mai und Juni 2020, liefert aber keinen Vergleich mit der Zeit vor der Pandemie. Es zeigt sich, dass während dieser Zeit die Mütter relativ häufig die Kinderbetreuung allein übernommen haben (zwischen 20 und 33 \% der Familien, je nach Gruppe). Väter beteiligten sich ebenfalls in vielen Familien an der Kinderbetreuung, jedoch selten allein (4-9\%), sondern meist gemeinsam mit der Mutter oder weiteren Personen. Auch dieser Studie zufolge hatte die berufliche Situation (v.a. die Möglichkeit des Homeoffice) einen entscheidenden Einfluss auf die Verteilung der Kinderbetreuung.

Auch eine Studie auf Basis der Böckler-Erwerbstätigenbefragung von April 2020 (Kohlrausch und Zucco 2020) bestätigt, dass Frauen in der Krise hauptverantwortlich für die Familienarbeit waren. So gaben unter den befragten Eltern in Partnerschaften 27 \% der Mütter, aber nur 16 \% der Väter an, dass sie die Arbeitszeit reduzieren mussten, um die Betreuung der Kinder sicherzustellen. Zudem berichtete die Mehrheit der befragten Mütter (54\%), in der Krise überwiegend die Kinderbetreuung zu übernehmen, verglichen mit $12 \%$ der Väter. Allerdings entspricht dieser Anteil bei den Vätern einer Verdopplung im Vergleich zum Niveau vor der Krise (6\%), während der Anteil bei den Müttern zurückging (von 61 \%).

Die Studie von Jessen et al. (2021) auf Basis von Daten der pairfam-Zusatzerhebung (Mai bis Juli 2020) deutet darauf hin, dass die Verteilung der Familienarbeit gerade in den Familien, die bereits vor der Krise eine relativ traditionelle Aufgabenteilung hatten, 
während der Pandemie noch ungleicher geworden ist. So reduzierte sich der Anteil der Familien, in denen die Frau überwiegend die Kinderbetreuung/Hausarbeit übernimmt, während der Krise deutlich zugunsten des Anteils der Familien, in denen die Frau diese Aufgaben (fast) vollständig übernimmt. Demgegenüber blieb der Anteil der Paare, die die Familienarbeit gleichmäßig aufteilten, auf konstantem Niveau.

Die Pandemie beeinflusste nicht nur die kurzfristige Arbeitsteilung in Familien, sondern nahm teilweise auch Einfluss auf die Geschlechterrollenvorstellungen, wie eine Studie von Danzer et al. (2021) auf Basis von Daten der ALLBUS- und der COMPASS-Befragungen zeigt. So gingen unter westdeutschen Vätern egalitäre Einstellungen zur Müttererwerbstätigkeit im Jahr 2021 merklich zurück. Damit ist für diese Gruppe eine Umkehr des vorherigen Trends in Richtung zunehmend egalitärer Einstellungen zu beobachten. Bei Müttern zeigten sich keine Einstellungsveränderungen.

\subsection{Elternspezifische Belastungen: Betreuung, Homeschooling und Sorgen um die Kinder}

Es liegt eine Reihe von Erkenntnissen zum Belastungsempfinden von Eltern in spezifischen Bereichen während der Krise vor. Der Fokus dieses Abschnitts liegt dabei auf den Belastungen durch Kinderbetreuung, Homeschooling und Sorgen um die Kinder, da diese Aspekte ausschließlich Eltern betreffen. ${ }^{6}$

Einen zumindest gedanklichen Vergleich zwischen der Belastung durch die Kinderbetreuung vor und während der Corona-Krise (im Mai 2020) bietet dabei die IAB-Befragung „Leben und Erwerbstätigkeit in Zeiten von Corona“ (Fuchs-Schündeln und Stephan 2020). Der Fokus liegt hier auf sozialversicherungspflichtig Beschäftigten im Alter von 18 bis 60 Jahren mit Kindern bis 18 Jahren im Haushalt. Dabei sagten $44 \%$ der Väter und 49 \% der Mütter, dass ihre Belastung durch Kinderbetreuung durch die Krise stark gestiegen sei. Weitere 31 \% der Väter und 25 \% der Mütter sagten, die Belastung sei etwas gestiegen. Der stärkere Anstieg der Belastung bei Müttern ließ sich dabei auch in einer multivariaten Regressionsanalyse bestätigen.

Auch die Allensbach-Studie (BMFSFJ 2020) hat Eltern zu ihren Erfahrungen mit der Organisation der Kinderbetreuung im ersten Lockdown befragt. Dabei zeigt sich, dass gut die Hälfte (55 \%) der Eltern mit Kindern unter 15 Jahren die Neuorganisation der Kinderbetreuung als schwierig empfand. Darunter befanden sich $20 \%$ Eltern, die die Neuorganisation schwierig fanden, und mit der Lösung nicht wirklich zufrieden waren, 
und weitere $35 \%$ der Eltern, die die Neuorganisation zwar schwierig fanden, aber eine gute Lösung gefunden haben. 26 \% der Eltern fanden die Neuorganisation der Kinderbetreuung nicht besonders bzw. überhaupt nicht schwierig, und die restlichen $19 \%$ mussten die Kinderbetreuung nicht neu organisieren.

In der gleichen Studie (BMFSFJ 2020) gaben zudem $23 \%$ der befragten Eltern an, die Betreuung zu Hause belaste sie oder den Partner bzw. die Partnerin stark. Ebenso sagten $23 \%$, ihr Leben sei stressiger geworden, weil sie sich neben dem Beruf und dem Haushalt auch noch um die Kinderbetreuung oder den Schulunterricht kümmern müssen. Insgesamt sagte weniger als die Hälfte (48 \%) der Eltern, dass die Kinderbetreuung zu Hause gut funktioniere und nur $19 \%$, dass sie die Kinder auch von zu Hause aus ganz gut fördern könnten. Demgegenüber machten sich $43 \%$ der befragten Eltern Sorgen, dass ihr Kind zu Hause nicht so gut gefördert werden könne wie in der Kita/Schule und später Nachteile haben könnte. Weitere 21 \% sorgten sich, Arbeit und Kinderbetreuung nicht gleichzeitig zu schaffen. Auch in der Studie „Familien und Kitas" empfanden viele Eltern die Vereinbarkeit von Familie und Beruf während des ersten Lockdowns als besonders herausfordernd (durchschnittlich 3,4 von 4 Punkten) (Cohen et al. 2020).

Ein etwas positiveres Bild liefert die SOEP-CoV-Studie mit Blick auf das Homeschooling während des ersten Lockdowns. So zeigten sich nur ca. 16 \% der Eltern während der Schulschließung damit überfordert, dafür zu sorgen, dass das Kind den Schularbeiten nachkommt (Zinn et al. 2020). Allerdings hatten einer anderen Studie zufolge ca. 30 \% der für das Homeschooling verantwortlichen Eltern das Gefühl, dass das Homeschooling die Beziehung zu ihrem Kind „ziemlich“ oder sogar „sehr“ belaste (Wildemann und Hosenfeld 2020).

Auch nach dem ersten Lockdown blieb die Belastung vor allem für Mütter weiterhin hoch, wie eine Forsa-Umfrage im Auftrag der Bepanthen-Kinderförderung zwischen Ende Oktober und Anfang November 2020 zeigt. Hier stimmten $57 \%$ der Mütter und 37 \% der Väter zu, mit den vielfältigen Anforderungen im Alltag überfordert zu sein, z. B. Kinderbetreuung, Haushalt und Berufsleben gleichzeitig zu managen (Bayer Vital 2021).

Die im Rahmen der CoronaCOMPASS-Studie erhobenen Daten vom Januar 2021 erlauben einen differenzierten Einblick in die Sorgen von Eltern während des zweiten Lockdowns. So macht sich jeweils über die Hälfte der Eltern um die Bildung (55\%) und die wirtschaftliche Zukunft ihrer Kinder (52 \%) große Sorgen, während die Gesundheit der Kinder mit $37 \%$ an dritter Stelle genannt wird und erst zuletzt Sorgen um die eigene 
wirtschaftliche Situation (28\%). Dabei schließen sich Sorgen um Bildung und Gesundheit der Kinder nicht aus, im Gegenteil: Eltern, die sich sehr um die Bildung der Kinder sorgen, sorgen sich gleichzeitig auch sehr um deren Gesundheit (52\% der Eltern, die sich um Bildung große Sorgen machen). Wie auch die Ergebnisse des DIW-FamilienMonitor_Corona bereits zeigen, sind Mütter stärker betroffen als Väter, ebenso Personen mit niedrigerer Bildung und niedrigerem Haushaltseinkommen (Huebener et al. 2021). Auch aus der Studie von Czymara et al. (2020) aus dem ersten Lockdown geht hervor, dass das Thema Kinderbetreuung die Eltern während der Pandemie stark beschäftigte; nur soziale Kontakte wurden von den Befragten noch häufiger erwähnt. Dabei zeigten sich deutliche Unterschiede zwischen den Geschlechtern: Frauen sorgten sich signifikant häufiger um Kinderbetreuung, während Männer sich häufiger um Erwerbsarbeit und die Wirtschaft Gedanken machten.

\subsection{Effekte der hohen Belastung auf das Wohlbefinden}

Eine anhaltend hohe psychische Belastung kann sich auf das Wohlbefinden der Eltern niederschlagen. In diesem Zusammenhang zeigen Studien auf Basis des NEPS (Zoch et al. 2020a) sowie des SOEP und der COMPASS-Befragung (Huebener et al. 2020) einen deutlichen Rückgang der Lebenszufriedenheit von Eltern zwischen der Vor-Corona-Zeit und dem ersten Lockdown. Beiden Studien zufolge fiel der Rückgang bei Müttern stärker aus als bei Vätern, was dem oben beschriebenen höheren Belastungsempfinden der Mütter entspricht. Zudem war der Rückgang der Lebenszufriedenheit laut Huebener et al. (2020) unter Eltern stärker als bei anderen Gruppen. Den stärksten Rückgang an Lebenszufriedenheit hatten Eltern mit kleinen Kindern zu verzeichnen - diejenige Gruppe, die vor der Krise eine besonders hohe Zufriedenheit aufwies.

Die Lebenszufriedenheit von Eltern veränderte sich auch über den weiteren Verlauf der Corona-Krise, wie der FamilienMonitor_Corona ebenfalls auf Basis der COMPASS-Daten zeigt (Abb. 19). Zunächst fällt auch hier wieder auf, dass die Lebenszufriedenheit der Mütter zu nahezu allen Messzeitpunkten niedriger liegt als die der Väter. Hinzu kommt, dass die Zufriedenheit der Mütter zwischen Mai 2020 und April 2021 mit Ausnahme eines kurzen Anstiegs im Februar/März stufenweise noch weiter abgenommen hat. Bei den Vätern hielt sich die Lebenszufriedenheit bis Januar 2021 auf einem relativ konstanten Niveau und fiel erst Ende Januar/Anfang Februar 2021, d. h. mit längerem Andauern des zweiten Lockdowns, deutlich ab. Zum Ende des Beobachtungszeitraums, im April 2021, war die durchschnittliche Lebenszufriedenheit unter Müttern und Vätern geringer als zu allen anderen Zeitpunkten der Befragung. 


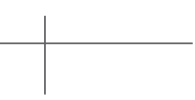

7,5

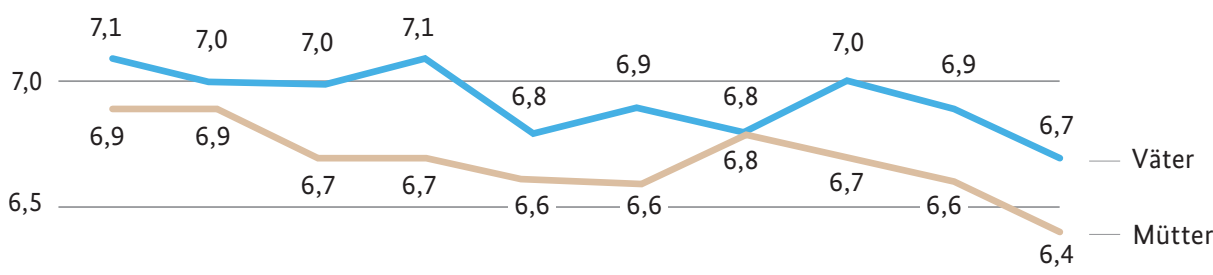

6,0

$\begin{array}{llllllllll}01.05 . & 01.06 . & 01.11 . & 07.01 . & 20.01 . & 03.02 . & 17.02 . & 03.03 & 17.03 . & 31.03 .\end{array}$

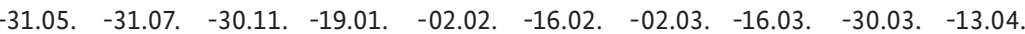

$\begin{array}{llllllllll}2020 & 2020 & 2020 & 2021 & 2021 & 2021 & 2021 & 2021 & 2021 & 2021\end{array}$

Abbildung 19: Entwicklung der Lebenszufriedenheit unter Eltern mit Kindern unter 16 Jahren, Mai 2020-April 2021 (Mittelwerte auf einer Skala von 0 bis 10)

Quelle: Eigene Darstellung, Datenbasis: FamilienMonitor_Corona (DIW und Infratest dimap 2021).

Einen direkten Zusammenhang zwischen Belastungsempfinden und Lebenszufriedenheit von Eltern stellt die IAB-Studie her (Fuchs-Schündeln und Stephan 2020). So haben diejenigen, die berichten, dass ihre Belastung durch Kinderbetreuung durch die Krise stark gestiegen ist, eine deutlich niedrigere mittlere Lebenszufriedenheit als diejenigen, die nicht von einem starken Anstieg der Belastung berichten. Die Lebenszufriedenheit von stark zusätzlich belasteten Müttern liegt bei 6,4 von 10 Punkten, während sie bei den anderen Müttern bei 7,3 Punkten lag. Bei den stark zusätzlich belasteten Vätern lag die Lebenszufriedenheit etwas höher als bei den entsprechenden Müttern (6,9 Punkte), allerdings nicht so hoch wie bei anderen Vätern (7,3 Punkte).

Die erhöhte Belastung von Müttern resultiert einer weiteren Studie zufolge auch in häufigerer Niedergeschlagenheit. So zeigen Bujard et al. (2020b) anhand von Daten der Mannheimer Corona-Studie auf, dass häufige Niedergeschlagenheit im April 2020 unter Müttern mit $17 \%$ deutlich weiter verbreitet war als unter kinderlosen Frauen (13\%). Bei Vätern (6\%) und kinderlosen Männern (8 \%) lag der Anteil deutlich niedriger. Ein grober Vergleich mit ähnlichen Daten aus dem Sozio-oekonomischen Panel von 2018, also zu Vor-Corona-Zeiten, weist dabei darauf hin, dass (nur) unter Müttern der Anteil derjenigen, die häufig niedergeschlagen sind, während der Krise zugenommen hat. Zudem weist die Studie von Calvano et al. (2021) auf einen leichten Anstieg an Depression und Ängstlichkeit bei Eltern während der Krise hin, wobei das Niveau bei Müttern auch hier signifikant höher lag als bei Vätern. 
Eine Tagebuchstudie über 21 Tage im ersten Lockdown belegt einen direkten Zusammenhang zwischen Homeschooling und elterlichem Befinden. So berichten Eltern an den Tagen, an denen sie stärker ins Homeschooling eingebunden waren, signifikant weniger positive Affekte (wie glücklich, fröhlich, ausgeglichen, entspannt sein) und mehr negative Affekte (wie ängstlich, wütend, traurig und besorgt sein) (Schmidt et al. 2021). Emotionale Erschöpfung ist eine konkrete mögliche Folge hoher Belastungen. In diesem Zusammenhang zeigt die Längsschnittstudie von Erwerbstätigen von Meyer et al. (2020), dass Frauen mit Vorschulkindern über den gesamten Studienzeitraum von April bis Juni 2020 stärker erschöpft waren als andere Gruppen. Darüber hinaus ist ersichtlich, dass für Mütter - und in geringerem Maße auch für Väter - die emotionale Erschöpfung dann besonders hoch war, wenn die Betreuungseinrichtungen geschlossen waren. Unter besonders starker Erschöpfung litten dabei Mütter, die von zu Hause aus arbeiteten, während die Betreuungseinrichtungen geschlossen waren. Der Konflikt zwischen Arbeit und Familie hatte dabei einen zentralen Einfluss auf das Niveau der emotionalen Erschöpfung. Auch in einer weiteren, multivariaten Analyse können die Autoren und Autorinnen bestätigen, dass die Kinderzahl in einem signifikant positiven Zusammenhang mit dem Erschöpfungsgrad Erwerbstätiger steht, und Frauen signifikant stärker erschöpft waren als Männer (Meyer et al. 2021).

In der DAK-Studie (Forsa 2020a) berichtet ebenfalls ein hoher Anteil der Eltern während des ersten Lockdowns von Erschöpfung und Müdigkeit; konkret waren 15 \% täglich und $31 \%$ mehrmals pro Woche erschöpft oder müde. Auch berichtet ein Teil der Eltern von Schlafproblemen und körperlichen Schmerzen. Im Geschlechtervergleich zeigt sich wieder, dass Mütter häufiger von all diesen Beschwerden berichten als Väter.

Einschränkungen des Wohlbefindens und der psychischen Gesundheit bei Eltern werden nicht nur von diesen selbst berichtet, sondern auch von Psychiaterinnen und Psychiatern sowie Psychotherapeutinnen und -therapeuten. So zeigt eine bundesweite Befragung dieser Expertengruppen im Oktober und November 2020 (Provona BKK 2020), dass psychische Beschwerden besonders unter Frauen und Familien mit Kindern im Haushalt zugenommen haben. $86 \%$ der befragten Fachleute sahen eine Zunahme von Beschwerden bei Frauen, $70 \%$ bei den Männern. Bei verheirateten Paaren mit Kindern nahmen $84 \%$ der Befragten eine Zunahme psychischer Probleme wahr und bei Alleinerziehenden sogar $92 \%$. Demgegenüber berichteten nur $49 \%$ der Befragten von einer Zunahme dieser Probleme bei verheirateten Paaren ohne Kinder. Nach Meinung der befragten Fachleute wirkten sich vor allem die soziale Isolation und eine angespannte familiäre Situation (z. B. durch familiäre Konflikte, Homeschooling und Homeoffice) negativ auf die psychische Gesundheit aus, gefolgt von räumlicher Enge zu Hause. 


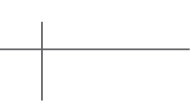

\title{
6.5 Risikofaktoren für hohe Belastung von Eltern während der Krise
}

\author{
GESCHLECHT
}

Wie oben beschrieben, zeigen diverse Studien während der Corona-Krise ein erhöhtes Belastungsniveau und ein niedrigeres Wohlbefinden von Müttern im Vergleich zu Vätern auf. Dies könnte darauf zurückzuführen sein, dass Mütter im Lockdown ebenso wie vor der Krise - den Hauptanteil der Haus- und Familienarbeit leisteten. Dies beinhaltet nicht nur die reine Zeitverwendung für diese Tätigkeiten (Kap. 6.2), sondern auch der sogenannte „Mental Load“, d. h. die kognitive Planungsarbeit dieser Aufgaben. Wie in Abschnitt 6.3 erwähnt, sorgten sich Frauen im ersten Lockdown signifikant häufiger um Kinderbetreuung als Männer. Bei Müttern kommt es durch Schul- und Kitaschließungen somit zu einer besonders starken Zusatzbelastung durch Hausarbeit, Kinderbetreuung und Homeschooling. Allerdings muss ebenfalls bedacht werden, dass Frauen auch vor der Krise bereits stärker von Stress, Depressionen und Erschöpfung betroffen waren als Männer (Kowal et al. 2020; Meyer et al. 2021).

\section{PARTNERSCHAFTSSTATUS}

Alleinerziehende sind aufgrund verschiedener Faktoren während der Krise in einer erschwerten Lage. Sie nutzen besonders häufig formelle Kinderbetreuung (Schober und Stahl 2014), Ganztagsschulen (Zinn et al. 2020) und informelle Betreuung durch Verwandte (Müller et al. 2020; Schober und Stahl 2014), so dass bei ihnen durch die Schul- und Kitaschließungen sowie die Reduktion der Kontakte zu den Großeltern eine besonders große Betreuungslücke entstanden ist. Alleinerziehende mussten sich dann häufig allein um den Erwerb des Lebensunterhalts, die Kinderbetreuung, das Homeschooling und den Haushalt kümmern. Dies galt vor allem zu Beginn des ersten Lockdowns, als Alleinerziehende häufig noch keinen Zugang zu Notbetreuung hatten. Doch auch Kinder in der Notbetreuung mussten zu Hause beim Lernen unterstützt werden (Zinn et al. 2020). Außerdem konnten Alleinerziehende im ersten Lockdown mit 33 \% seltener im Homeoffice arbeiten als Eltern in Paarfamilien mit 38 \% (Zinn et al. 2020). Erschwerend hinzu kommt, dass Alleinerziehende tendenziell ein niedrigeres Einkommen und ein höheres Armutsrisiko als Paarfamilien aufweisen, was einen weiteren Risikofaktor darstellt.

Entsprechend finden mehrere Studien ein erhöhtes Belastungsniveau Alleinerziehender im ersten Lockdown: Nach Daten der Böckler-Erwerbstätigenbefragung waren alleinerziehende Mütter im April 2020 mit $60 \%$ besonders häufig durch die Gesamtsituation belastet, verglichen mit 49 \% der Mütter in Partnerschaften und 45 \% der Väter in 
Partnerschaften (Bujard et al. 2020b). Auch durch die Anforderungen des Homeschoolings zeigen sich Alleinerziehende stärker belastet als Paareltern. So stimmen in der SOEP-CoV-Studie nur $6 \%$ der Eltern in Paarbeziehungen, aber $17 \%$ der Alleinerziehenden der Aussage voll zu, dass es sie überfordern wird, dafür zu sorgen, dass das Kind den Schularbeiten nachkommt. Innerhalb der Gruppe der Alleinerziehenden sind es dabei vor allem die Erwerbstätigen und diejenigen mit niedrigem Bildungsniveau, die sich durch das Homeschooling überfordert fühlen (Zinn et al. 2020). Ähnlich zeigt die Allensbach-Studie, dass sich vor allem Alleinerziehende sorgen, dass ihr Kind zu Hause nicht so gut gefördert werden kann wie in der Kita/Schule und dass die Kinder später Nachteile haben könnten. Außerdem sorgt sich ein überdurchschnittlich hoher Anteil Alleinerziehender, Arbeit und Kinderbetreuung nicht gleichzeitig zu schaffen. Auch die Neuorganisation der Kinderbetreuung empfanden Alleinerziehende häufiger als schwierig (BMFSFJ 2020). Ebenso zeigen Langenkamp et al. (2020), dass vor allem Alleinerziehende sich häufig um die Kinderbetreuung sorgten, oft in Kombination mit finanziellen Sorgen. Zu einem etwas anderen Ergebnis kommt allerdings die IAB-Studie: Hier ist der Anteil der Alleinerziehenden, die berichten, die Belastung durch Kinderbetreuung sei während der Krise stark gestiegen, niedriger als bei den Eltern in Partnerschaften (Fuchs-Schündeln und Stephan 2020). Die Autoren der Studie führen dies darauf zurück, dass Alleinerziehende relativ schnell Zugang zu Notbetreuung erhielten.

\section{MERKMALE DER KINDER}

Mit der Anzahl der Kinder steigt im Lockdown der Bedarf an Betreuung, Beschulung und das Aufkommen an Hausarbeit. Die wenigen bisher vorliegenden Studien zum Zusammenhang von Kinderzahl und elterlicher Belastung zeichnen allerdings ein uneinheitliches Bild. So scheinen Mehrkinderfamilien laut deskriptiven Ergebnissen der für alle Eltern mit Kindern unter 15 Jahren repräsentativen Allensbach-Studie nicht schlechter mit der Betreuung und Förderung von Kindern zurechtzukommen als andere. 50 \% der befragten Eltern in Mehrkinderfamilien sagen, dass die Kinderbetreuung zu Hause gut funktioniert, verglichen mit einem Durchschnitt von $48 \%$. Ebenso sind $22 \%$ der Eltern von Mehrkinderfamilien der Meinung, dass sie die Kinder auch von zu Hause aus ganz gut fördern können, verglichen mit 19 \% aller Eltern. Ein anderes Bild zeigt sich allerdings in multivariaten Studien, die ausschließlich auf Erwerbstätige fokussieren. So findet die IAB-Studie in einer Regressionsanalyse, dass der Anteil derjenigen, die von einer stark gestiegenen Belastung durch Kinderbetreuung berichten, mit der Kinderzahl steigt (Fuchs-Schündeln und Stephan 2020). Ebenso zeigen die Regressionen in der Beschäftigtenstudie von Meyer et al. (2021) einen positiven Zusammenhang zwischen Kinderzahl (sowohl mit Blick auf Vorschul- als auch Schulkinder) und emotionaler Erschöpfung auf. 
Auch der Gesundheitszustand der Kinder ist von Relevanz. Im ersten Lockdown entfielen viele Therapien, Hilfen und unterstützende Maßnahmen für Kinder mit chronischen Erkrankungen oder Behinderungen. Entsprechend zeigt eine Studie des Universitätsklinikums Freiburg und des Kindernetzwerks, dass Eltern dieser Kinder durch die Covid-19-Pandemie häufiger psychisch belastet sind und ein deutlich höheres Risiko für eine depressive Erschöpfung haben als Eltern gesunder Kinder (Langer und Barth 2020).

\section{FINANZIELLE SITUATION}

Ein niedriges Einkommen kann mit verschiedenen Belastungsfaktoren einhergehen, wie z. B. beengte Wohnverhältnisse oder eine schlechte technische Ausstattung für das Homeoffice und Homeschooling. Entsprechend zeigt die österreichische SORA-Studie, dass der Anteil der belasteten Eltern mit steigendem Haushaltseinkommen abnimmt: Fühlten sich im November 202064 \% der Eltern in Haushalten mit einem Einkommen bis $3000 €$ durch die Situation belastet, so lag dieser Anteil bei Haushalten mit einem höheren Einkommen bei 51 \%. In beiden Gruppen ist jedoch der Anteil seit dem ersten Lockdown im April 2020 angestiegen, um jeweils 11 Prozentpunkte. Ähnlich zeichnet sich in der Allensbach-Studie für Eltern mit hohem sozio-ökonomischen Status eine geringere Belastung ab: So sagten $66 \%$ der Eltern mit hohem Status, 61 \% derjenigen mit mittlerem Status, aber nur $49 \%$ derjenigen mit niedrigem Status, dass sie bisher ganz gut durch die Krise gekommen seien (BMFSFJ 2020).

Auch die Studie „Familien und Kitas“ findet Unterschiede in der Bewältigung der Corona-Krise nach finanzieller Situation. So berichteten Eltern mit finanziellen Sorgen häufiger über negative Aspekte der Corona-Zeit als Eltern ohne finanzielle Sorgen. Zum Beispiel stimmten sie eher der Aussage zu, dass sie sich den neuen Aufgaben und Anforderungen oft nicht gewachsen fühlen, und dass sie sich durch die Vielzahl an Belastungen häufig gestresst fühlen (Cohen et al. 2020). Keinen Unterschied in der Zunahme der Belastung durch Kinderbetreuung nach dem Einkommen findet allerdings die IAB-Studie (Fuchs-Schündeln und Stephan 2020).

\section{ERWERBSSTATUS}

Je höher die Wochenarbeitszeit, desto weniger zeitliche Ressourcen stehen Eltern für Betreuung und Homeschooling zur Verfügung. Entsprechend deuten einige Studien darauf hin, dass die Belastungen während der Krise für vollzeitbeschäftigte Eltern besonders hoch sind. So fand die Studie „Familien und Kitas“, dass vollzeitbeschäftigte Eltern eher den Aussagen zustimmten, sich den neuen Aufgaben und Anforderungen 
nicht gewachsen zu fühlen und durch die Vielzahl an Belastungen häufig gestresst zu sein. Bei Teilzeitbeschäftigten war die Zustimmung etwas geringer und bei Nichterwerbstätigen am geringsten. Nichterwerbstätige Eltern waren dafür allerdings durch das ständige „Aufeinanderhocken“ häufiger nervlich belastet (Cohen et al. 2020). Speziell für die Gruppe der Alleinerziehenden fand die SOEP-CoV-Studie ebenfalls heraus, dass Erwerbstätige stärker durch die Anforderungen des Homeschoolings belastet waren als Nichterwerbstätige (Zinn et al. 2020). Innerhalb von Paarfamilien scheint es demgegenüber auf die Erwerbskonstellation anzukommen: Vor allem Paare, in denen beide Elternteile mindestens 25 Stunden pro Woche erwerbstätig waren, hatten Schwierigkeiten mit der Neuorganisation der Kinderbetreuung im ersten Lockdown und fanden besonders häufig keine zufriedenstellende Lösung (BMFSFJ 2020).

\section{ARBEITSORT}

Viele Eltern arbeiteten während des ersten Lockdowns von zu Hause aus, um die Betreuung ihrer Kinder sicherzustellen. Allerdings war dieses Arrangement nicht mit geringeren Belastungen verbunden. Im Homeoffice mussten Eltern oft gleichzeitig Erwerbsarbeit und Betreuung bzw. Homeschooling bewerkstelligen, während Beschäftigte, die vor Ort beim Arbeitgeber arbeiteten, die Betreuung der Kinder einer anderen Person (z. B. Partner bzw. Partnerin, Notbetreuung) überlassen konnten.

Entsprechend stimmten in der Studie „Familien und Kitas" Eltern im Homeoffice eher als Eltern, die nicht im Homeoffice arbeiteten, den Aussagen zu, dass sie sich den Aufgaben und Anforderungen oft nicht gewachsen fühlten, dass die Vereinbarkeit von Familie und Beruf zurzeit besonders herausfordernd sei, und dass sie sich durch die Vielzahl an Belastungen häufig gestresst fühlten (Cohen et al. 2020). Auch die IAB-Befragung weist auf besondere Belastungen der Eltern im Homeoffice hin. So sagten 54 \% der Väter und 61 \% der Mütter, die (zumindest teilweise) im Homeoffice arbeiteten, dass ihre Belastung durch die Kinderbetreuung durch die Corona-Krise stark gestiegen sei. Unter allen erwerbstätigen Eltern waren dies nur $44 \%$ der Väter und $49 \%$ der Mütter. Der Unterschied zwischen Eltern im Homeoffice und denjenigen, die nicht im Homeoffice arbeiteten, trat dabei auch in einem multivariaten Regressionsmodell deutlich hervor (Fuchs-Schündeln und Stephan 2020). Auch zeigte sich ein stärkerer Konflikt zwischen Privat- und Arbeitsleben sowie ein höheres Niveau emotionaler Erschöpfung unter Beschäftigten, die ganz im Homeoffice arbeiteten, verglichen mit Beschäftigten, die nicht oder nur teilweise im Homeoffice arbeiteten (Meyer et al. 2020; Meyer et al. 2021). 


\section{BILDUNGSNIVEAU}

Einige wenige Studien weisen auch auf einen Einfluss des Bildungsniveaus auf das elterliche Belastungsempfinden hin. Die SOEP-CoV-Studie zeigt auf, dass sich Eltern mit niedrigerem Bildungsniveau stärker durch das Homeschooling belastet fühlten als Eltern mit hohem Bildungsniveau. Dies könnte darauf zurückzuführen sein, dass Eltern mit niedrigem Bildungsniveau tendenziell eine geringere Nähe zu Schule und Schulstoff aufweisen als höhergebildete Eltern (Zinn et al. 2020). Demgegenüber berichteten in der IAB-Befragung besonders Akademikerinnen und Akademiker von einem starken Anstieg der Kinderbetreuungsbelastung durch die Krise (Fuchs-Schündeln und Stephan 2020). Wenngleich die Autorinnen selbst keine Erklärung hierfür anbieten, könnte dieses Ergebnis darauf zurückzuführen sein, dass Eltern mit hohem Bildungsniveau vor der Krise besonders häufig Kindertagesbetreuung in Anspruch nahmen (Schober und Stahl 2014) und Hochqualifizierte generell zudem eher im Homeoffice arbeiteten (Bujard et al. 2020b). 

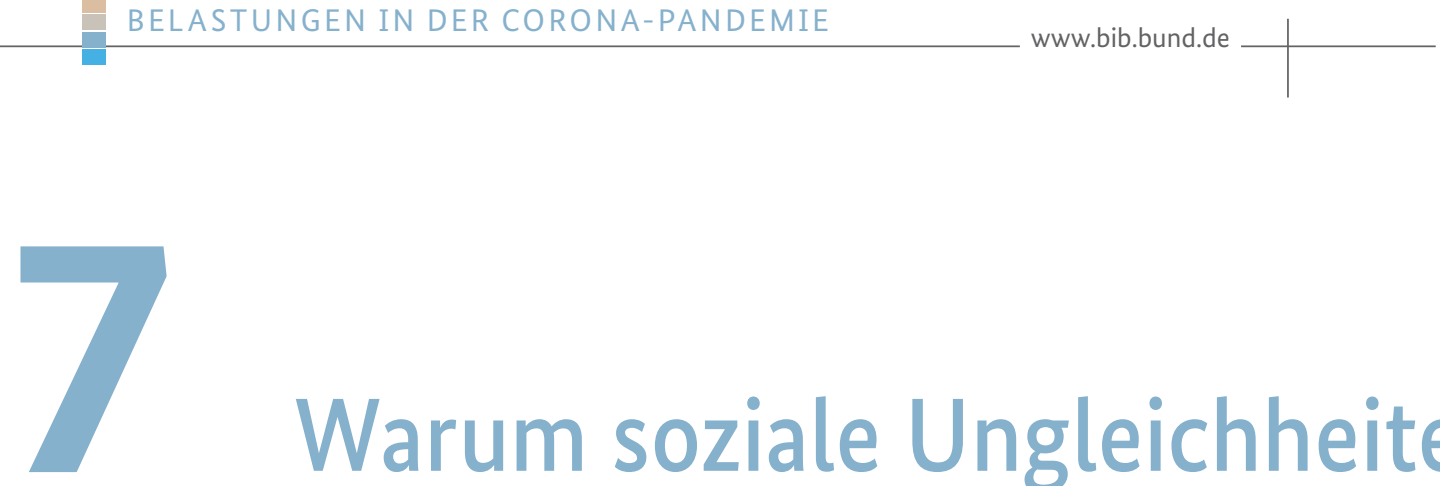

\section{Warum soziale Ungleichheiten die Belastungen verstärken}

Das Thema soziale Ungleichheiten zieht sich durch alle vorhergehenden Themen, von Bildung über körperliche und psychische Gesundheit bis hin zur Persönlichkeitsentwicklung. Kinder und Jugendliche, die bereits vor der Pandemie Schwierigkeiten hatten, sind von den neuen Problemlagen besonders betroffen, während Kinder, die auch vorher gut zurechtkamen, die Situation relativ gut meistern. So zeigt die DJI Studie „Kindsein in Zeiten von Corona“, dass fast $70 \%$ der Eltern insgesamt der Meinung sind, dass ihr Kind mit der aktuellen Situation gut zurechtkommt (Langmeyer et al. 2020). Insgesamt $30 \%$ nehmen Probleme wahr, insofern spricht dies gegen die These einer verlorenen Generation. Dabei zeigt sich, dass es erhebliche soziale Ungleichheiten gibt, das bedeutet: Die Probleme manifestieren sich besonders in bestimmten Bevölkerungsgruppen. Von Lehrkräften über Gesundheitsexpertinnen und -experten bis hin zu Kinderpsychologinnen und -psychologen besteht die Sorge, dass Kinder in besonderen Problemlagen noch weiter abgehängt werden als vor der Pandemie. Im Folgenden wird die Rolle sozialer Ungleichheiten anhand der Beispiele Bildung und Wohnsituation verdeutlicht.

\subsection{Soziale Ungleichheiten bei Bildung und Förderung}

Soziale Ungleichheiten zeigen sich z. B. beim Homeschooling, für das die Eltern eine entscheidende Rolle spielen. Ergänzend zu digitalen Unterrichtsformen übernehmen sie häufig ein Stück weit die Rolle der Lehrperson, indem sie für eine adäquate Lernumgebung und einen strukturierten Tagesablauf sorgen sowie Aufgaben erklären. Diese elterliche Unterstützung unterscheidet sich (1) aufgrund der verfügbaren digitalen Infrastruktur, (2) der Zeitkapazitäten und Bildungshintergründe der Eltern und (3) des Unterstützungsbedarfs seitens der Kinder.

Für die Lernerfolge sind insbesondere dort Herausforderungen zu erwarten, wo Eltern ihre Kinder aufgrund einer nicht deutschen Familiensprache und/oder einer geringen 
eigenen Bildung nur wenig beim Heimunterricht unterstützen können. Hier soll zunächst die Größenordnung dieser Gruppe dargestellt werden, um ihre Bedeutsamkeit zu unterstreichen: Bei etwa 14 bis 17 \% der Schulkinder wird zuhause überwiegend nicht Deutsch gesprochen. Angesichts von 7,3 Mio. Schülerinnen und Schülern der Klassen 1-10 in Deutschland, entspricht dies etwa einer Millionen Schulkinder, die zuhause überwiegend nicht Deutsch sprechen. Viele davon waren während der Lockdowns mehrere Monate nicht in der Schule. Wie bereits dargestellt, ist für diese Kinder eine sprachliche Frühförderung bereits im Vorschulalter und das alltägliche Üben in der Schule besonders wichtig für den akzentfreien Spracherwerb (Leopoldina 2021). Bei rund $11 \%$ haben beide Eltern einen geringen Bildungsabschluss. Bei 2-3\% der Schulkinder kommen beide Faktoren zusammen (Abb. 20). Bereits vor der Corona-Krise waren in Deutschland Bildungserfolge stark vom Bildungshintergrund der Eltern abhängig. Im aktuellen Heimunterricht wird dieser Aspekt noch verstärkt, da die Eltern oft die einzigen stets verfügbaren erwachsenen Ansprech- und Unterstützungspersonen für Schüler und Schülerinnen sind. Der weitere Ausbau von interaktiven digitalen Bildungsangeboten kann diesen Tendenzen zumindest etwas entgegenwirken. Besondere Unterstützung durch zielgruppenspezifische Förderung benötigen Kinder von Eltern mit niedriger Bildung bzw. deren Muttersprache nicht Deutsch ist.

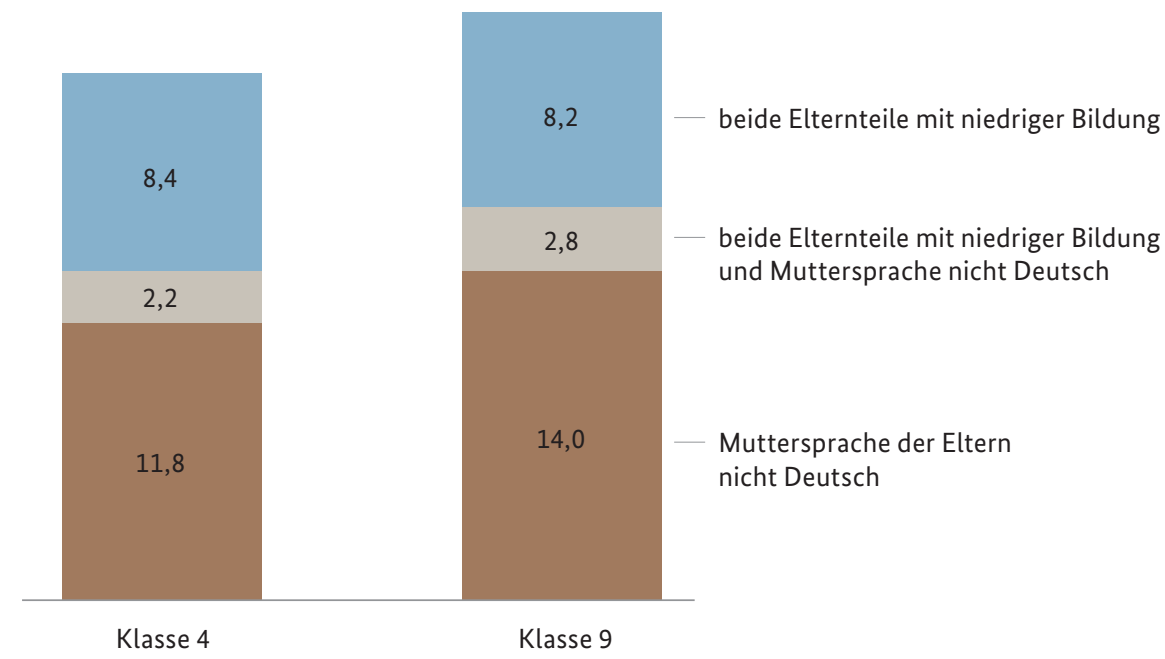

Abbildung 20: Schülerinnen und Schüler aus bildungsfernen oder von anderer Muttersprache geprägten Familien (Anteile in \%)

Quelle: Datenquelle: IQB-Bildungstrend 2016 (Klasse 4) und 2018 (Klasse 9), Berechnungen: IQB und BiB. 
Im ifo-Bildungsbarometer wurden Eltern um ihre Einschätzung gebeten, wie sie die Situation des Lernens zuhause bewerten. Fast zwei Drittel (64 \%) waren der Meinung, dass ihr Kind „viel weniger" gelernt habe. Dabei zeigen sich Unterschiede zwischen Eltern mit und ohne akademischem Hintergrund: Nicht-Akademiker bzw. Nicht-Akademikerinnen sehen größere Lerneinbußen und bewerten auch das Lernumfeld zu Hause schlechter als Eltern mit einem akademischen Abschluss (Abb. 21).

\subsection{Soziale Ungleichheiten bei der Wohnsituation}

Die Schließungen von Schulen und Kitas sowie von Spielplätzen, Sportplätzen und Freizeitstätten waren mit massiven Einschnitten im Alltag vieler Kinder und Jugendlicher verbunden. Aber auch Erwachsene waren von einer eingeschränkten Bewegungsfreiheit im öffentlichen Raum betroffen, da die meisten Sportangebote, vor allem Gruppen- und Mannschaftssport, lange Zeit nicht stattfinden konnten. Dadurch verbrachten Familien den größten Teil ihrer Zeit in der Wohnung; dies galt für die Kinder, aber auch für die Eltern, die im Homeoffice arbeiteten. Hier war die Wohnsituation von entscheidender Bedeutung.

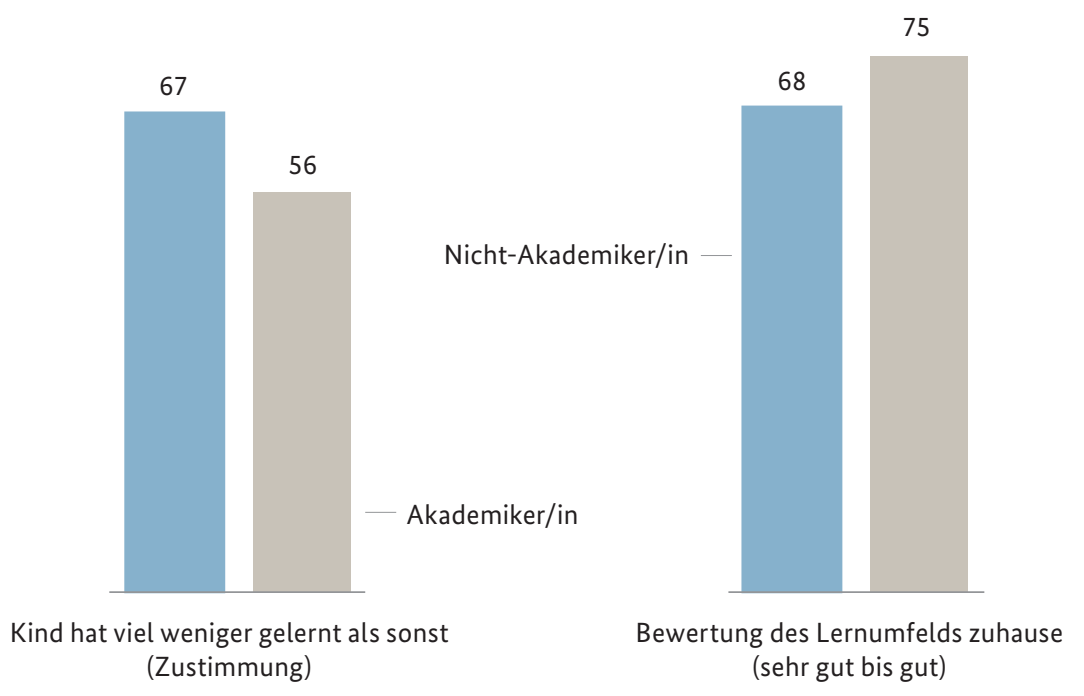

Abbildung 21: Wie bewerten Eltern die Zeit der Schulschließung? (Angaben in \%) 
Vor allem in Großstädten ist die Wohnsituation vieler Familien beengt. So lebt mehr als die Hälfte der Familien mit zwei Kindern in Großstädten in einer Wohnung, die kleiner als 100 Quadratmeter ist, $26 \%$ sogar in Wohnungen mit weniger als 80 Quadratmetern (Abb. 22). In städtischen Kreisen und auf dem Land wohnt nur etwa jede vierte Familie mit zwei Kindern in einer Wohnung, die kleiner als 100 Quadratmeter ist.

Durch die Kontaktsperre, vermehrte Homeoffice-Nutzung und Schließung von Bildungs- und Sportstätten war nicht nur die Bewegung eingeschränkt, sondern auch die Möglichkeiten reduziert, sich an der frischen Luft zu bewegen. Dies kann sich negativ auf die psychische und körperliche Gesundheit auswirken. Möglichkeiten der Gartennutzung wurden elementar wichtig, denn Bewegung im Garten kann dies zumindest ein Stück weit kompensieren. Von den etwa 8,05 Mio. Familien mit Kindern unter 18 Jahren haben 5,42 Mio. eine Wohnsituation mit Gartennutzung (67,3 \%, Abb. 23). Dagegen haben 2,63 Mio. Familien mit Kindern keine Gartennutzung; die meisten davon wohnen in Mehrfamilienhäusern (2,44 Mio.). In besonderem Maße sind Alleinerziehende von Wohnsituationen ohne Garten betroffen. Während mehr als zwei Drittel der Paarfamilien (71,1 \%) über Gartennutzung verfügen, trifft dies nur auf knapp die Hälfte $(49,4 \%)$ der Familien von Alleinerziehenden zu.

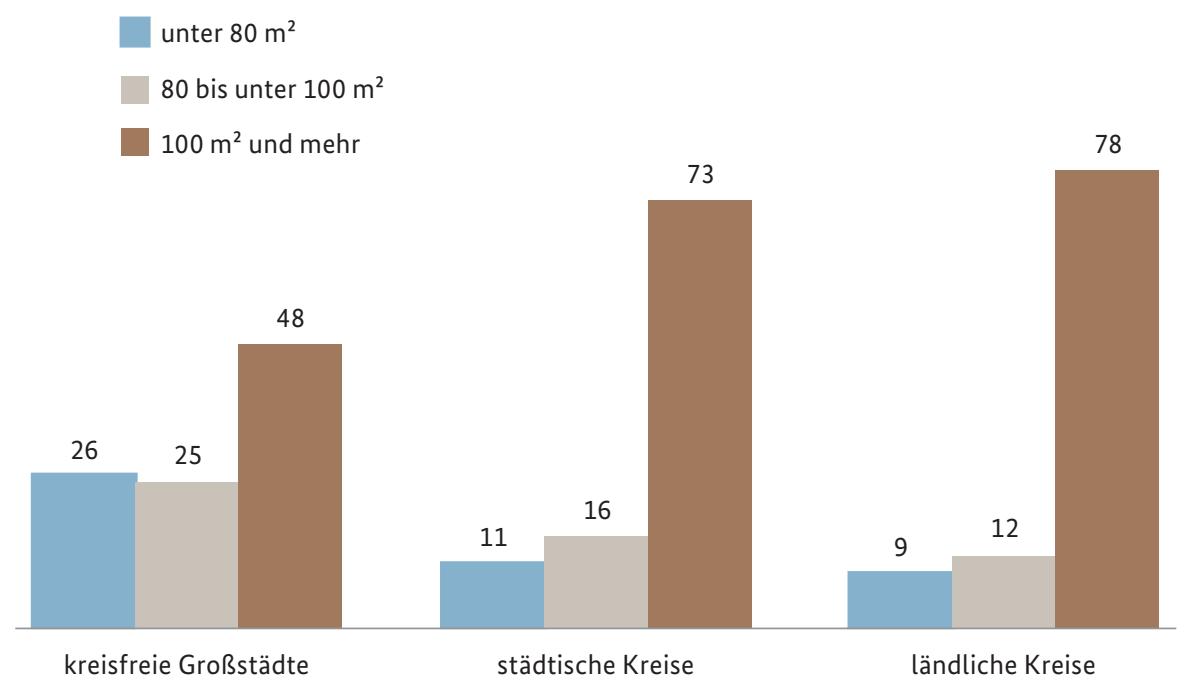

Abbildung 22: Wohnsituation von Familien mit zwei Kindern nach Regionstyp (Anteile in \%)

Quelle: Mikrozensus 2018, Sonderauswertung Statistisches Bundesamt, Berechnung BiB. 
In der COPSY-Studie konnte schließlich auch gezeigt werden, dass Kinder und Jugendliche mit mehreren Risikofaktoren, wie einem schlechten Familienklima im Elternhaus und gleichzeitig mit Eltern mit einem niedrigen Bildungsabschluss oder mit Migrationshintergrund oder beengtem Wohnraum (< 20 qm Wohnfläche/Person), die Veränderungen durch die Pandemie als besonders belastend erleben. Sie wiesen eine deutlich stärkere Belastung durch die Pandemie auf ( 43 \% zu 27 \%), häufigere psychosomatische Beschwerden, eine deutlich geminderte Lebensqualität sowie ausgeprägtere Symptome von Angst und Depressivität.

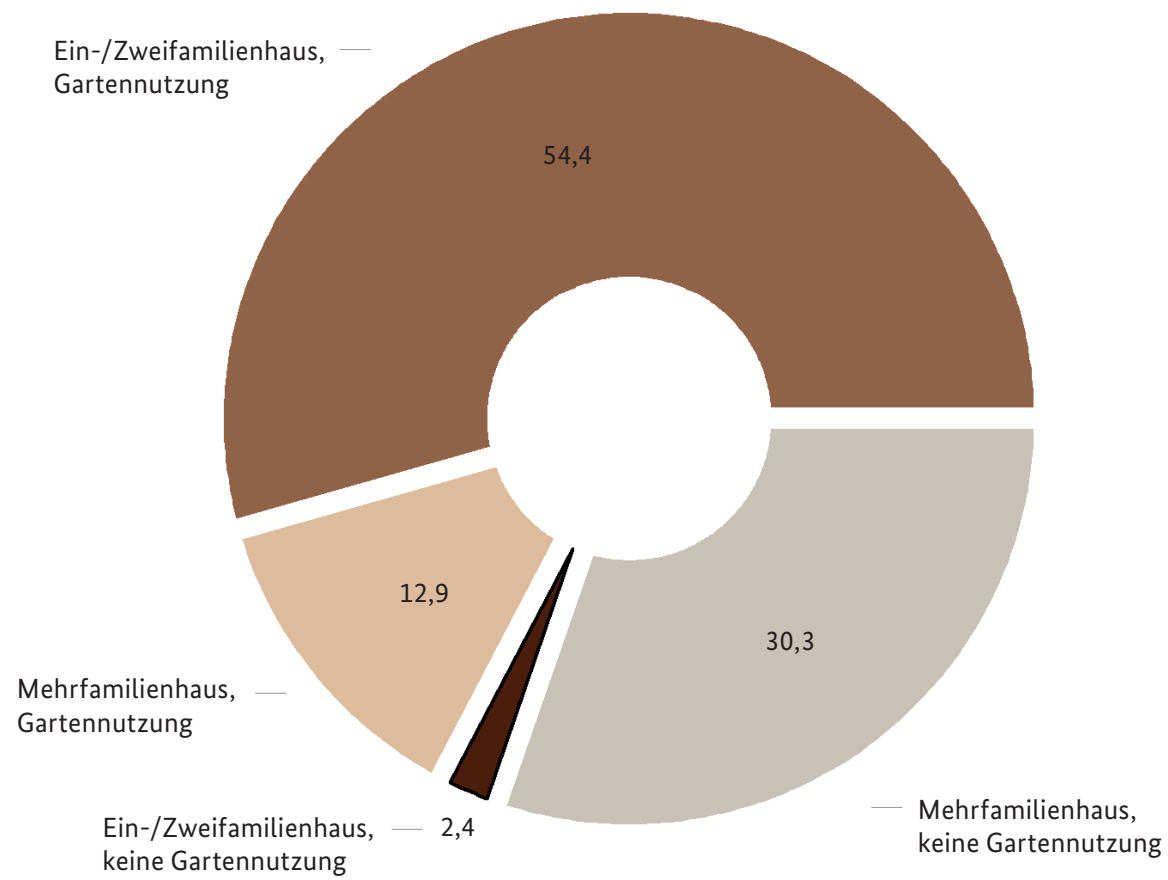

Abbildung 23: Möglichkeiten der Gartennutzung von Familien mit minderjährigen Kindern (Anteile in \%)

Quelle: Eigene Berechnung und Darstellung auf Basis von SOEP v35, 2018. 


\section{Fazit und Empfehlungen}

\subsection{Zentrale Ergebnisse}

Weitreichende Auswirkungen der Kita- und Schulschließungen auf Bildung, Gesundheit und Lebensqualität

Die Corona-Pandemie und die damit verbundenen Kita- und Schulschließungen haben weitreichende Auswirkungen auf Bildung, Gesundheit, Lebensqualität und Zukunftsperspektiven von Familien. Psychosoziale und gesundheitliche Beeinträchtigungen haben zugenommen und sollten umfangreich bei politischen Entscheidungen berücksichtigt werden. Familien haben in der Pandemie sehr viel geleistet. Viele sind relativ gut durch diese Zeit gekommen, andere haben vielfältige Beeinträchtigungen erfahren und befinden sich hinsichtlich Gesundheit, Bildung oder auch finanziell in schwierigen Lebenslagen.

\section{Mio. Kinder und Jugendliche besuchen Kitas oder Schulen}

Insgesamt besuchen etwa 11 Mio. Kinder und Jugendliche Kitas oder Schulen. Etwa 2,9 Mio. Kinder unter 6 Jahren gehen in Kitas oder Tagespflege. Rund 3 Mio. Kinder besuchen die Grundschule, 5,1 Mio. Kinder bzw. Jugendliche die Sekundarstufe, also die Klassen 5 bis 13. Etwa 14,6 Mio. Eltern leben mit minderjährigen Kindern zusammen, davon 13,1 Mio. Paare und 1,5 Mio. Alleinerziehende. Die Gruppen, die hier näher betrachten wurden, stellen somit einen großen Teil der Bevölkerung dar.

Lernzeit und Lernerfolg haben sich in den Schulschließungsmonaten deutlich reduziert Belastungen für Kinder und Jugendliche betreffen Bildung, körperliche Gesundheit, psychische Gesundheit und die Persönlichkeitsentwicklung. Studien zeigen, dass sich die Zeit für schulische Aktivitäten während des ersten Lockdowns halbiert hat und im zweiten Lockdown bei 59 \% lag, d. h. dass es umgekehrt $40 \%$ weniger Schul- und Lernzeit im Durchschnitt gab. Umgerechnet auf die Dauer der Schulschließungen bedeutet dies, bei zweieinhalb Monaten Schulschließung ein Monat und bei fünf Monaten Schulschließung zwei Monate weniger Schul- und Lernzeit. Auch der wahrgenommene sowie der getestete 
Lernerfolg hat sich reduziert. Hinter den durchschnittlichen schulischen Rückständen gibt es erhebliche Unterschiede zwischen den Schülerinnen und Schülern, einige konnten im Distanzunterricht relativ gut lernen, andere sind besonders stark abgehängt.

Gesundheitliche und entwicklungspsychologische Dimension von enormer Bedeutung Die Auswirkungen der Pandemie auf Kinder und Jugendliche sind vielfältig. Besonders die Folgen der Schulschließungen standen anfangs in ihrer gesundheitlichen und entwicklungspsychologischen Dimension weniger im Fokus. Es gibt Hinweise auf einen Anstieg von psychischen Beeinträchtigungen von Kindern, insbesondere bei psychosomatischen Beschwerden, Verhaltensauffälligkeiten und psychischen Erkrankungen, vor allem bei bereits vorbelasteten Kindern. Die Persönlichkeits- und Identitätsentwicklung vieler Kinder und Jugendlicher werden durch die Kontaktbeschränkungen beeinträchtigt. Gerade bei Entwicklungsübergängen ist die Zunahme von Kontaktängsten problematisch. Hochgerechnet hätte sich infolge der Pandemie und der damit verbundenen Schulschließungen bei 1,7 Mio. 11- bis 17-Jährigen die gesundheitsbezogene Lebensqualität erheblich verschlechtert.

\subsection{Jugendliche im Alter von 16 bis 19 Jahren mit Depressivitätssymptomatik} Unsere Analysen des deutschen Familienpanels pairfam weisen darauf hin, dass etwa 25 \% der Jugendlichen eine klinisch relevante Symptomatik von Depressivität im Mai/ Juni 2020 nach dem ersten Lockdown aufweisen. Empirische Grundlage ist hier keine Diagnose, sondern eine Selbsteinschätzung nach der international in der Psychologie anerkannten Skala „State and Trait Depression Scale“. Im Jahr vor der Pandemie betraf das lediglich 10 \% der Jugendlichen. Besonders gefährdet für psychische Probleme sind weibliche Jugendliche, hier hat sich die subjektive Depressivitätssymptomatik von 13 auf $35 \%$ fast verdreifacht. Auch Jugendliche mit Migrationshintergrund sind besonders betroffen. Hochgerechnet würde der Anstieg der Depressivitätssymptomatik 477.000 Jugendlichen im Alter von 16 bis 19 Jahren entsprechen.

\section{Keine verlorene Generation, große Unterschiede in den Auswirkungen}

Zum gegenwärtigen Zeitpunkt und angesichts unterschiedlicher Betroffenheit ist es nicht gerechtfertigt, von einer „verlorenen Generation“ zu sprechen. Mehrere Studien weisen darauf hin, dass rund zwei Drittel der Kinder und Jugendlichen trotz mancher Schwierigkeiten relativ gut durch die bisherigen pandemiebedingten Einschränkungen gekommen sind. Es ist zu erwarten, dass in der Phase auch Kompetenzen hinsichtlich Digitalisierung und Selbständigkeit erworben wurden. Eine differenzierte Sicht ist notwendig, um die belasteten Kinder und Jugendlichen zu identifizieren. Diese finden sich in allen Bevölkerungsgruppen, jedoch sind manche soziodemografischen Gruppen besonders belastet. 


\section{Mehr Bildschirmzeit und weniger Sport}

Die Zeit für Sport hat sich reduziert, da Schulsport und Vereinssport lange Zeit untersagt waren. Im ersten Lockdown wurde durchschnittlich täglich 11 Minuten weniger Sport getrieben. Für den zweiten Lockdown liegen (noch) keine Daten vor, es ist jedoch aufgrund der Jahreszeit von einem stärkeren Rückgang an Sport und gleichzeitig stärkeren Anstieg der Bildschirmzeit auszugehen. Auch haben Ernährungsprobleme und Gewalterfahrungen zugenommen.

\section{Väter in Kurzarbeit und im Homeoffice haben viel zusätzliche Familienarbeit übernommen und den durchschnittlichen Gender-Care-Gap - zumindest vorübergehend - reduziert}

Eltern waren Studien zufolge während der Corona-Krise stärker belastet als zuvor und stärker als kinderlose Personen. Besonders im Bereich Kinderbetreuung und Homeschooling standen durch die Schließungen von Kitas und Schulen viele Eltern vor großen Herausforderungen. Der zusätzliche Zeitbedarf wurde von Vätern und Müttern je nach beruflicher Situation unterschiedlich aufgefangen. Viele Väter haben sich in der Familie zusätzlich engagiert, vor allem in Kurzarbeitsphasen. Dies ist ein Grund dafür, dass sich im ersten Lockdown der durchschnittliche Anteil der Familienarbeit der Väter erhöht hat. Mütter jedoch übernahmen nach wie vor den Hauptteil der Familienarbeit.

Mütter haben die meiste Familienarbeit geleistet, teilweise emotionale Erschöpfung Auch der Mental Load, d. h. die kognitive Planungsarbeit der Familienaufgaben, war bei Müttern besonders hoch. Diese Belastungen haben sich zudem auf verschiedene Aspekte des Wohlbefindens, z. B. auf die Lebenszufriedenheit und die emotionale Erschöpfung, ausgewirkt. Dabei existieren verschiedene Risikofaktoren, die eine erhöhte Belastung bedingten. Insbesondere Mütter, Alleinerziehende und Familien mit niedrigem Einkommen waren besonders betroffen.

\section{Soziale Ungleichheiten verstärken die Belastung von Familien, vor allem bei Bildung und Wohnsituation}

Schülerinnen und Schüler aus bildungsfernen Familien oder die zuhause kein Deutsch sprechen, sind durch Schulschließungen besonders benachteiligt. Bei etwa $11 \%$ der Schulkinder haben beide Eltern einen geringen Bildungsabschluss. Bei 14 bis $17 \%$ wird zuhause überwiegend nicht Deutsch gesprochen, das entspricht rund einer Mio. Schulkinder. Zusätzlich ist bei Kontaktbeschränkungen die eigene Wohnsituation umso wichtiger: Ein Drittel der Familien hat keine Gartennutzung; die meisten davon wohnen in Mehrfamilienhäusern. Gerade in Großstädten leben viele Familien beengt; so leben 26 \% der Familien mit zwei Kindern in einer Wohnung mit weniger als 80 Quadratmetern. 


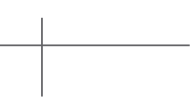

\subsection{Wissenschaftliche Empfehlungen}

\section{Kitas und Schulen sollten prioritär offengehalten werden}

Gegenwärtig existiert eine komplexe Kombination aus Schul- und Lernzeitrückständen und teils erheblichen psychischen Belastungen für eine größere Zahl von Kindern und Jugendlichen. Auch Eltern sind teilweise erheblich belastet infolge der monatelangen Mehrfachbelastung aus Beruf, Betreuung und Beschulung der Kinder sowie der Organisation der Familie in der Pandemie. Insbesondere aufgrund der erheblichen Größenordnung der psychisch-gesundheitlichen Auswirkungen der Schulschließungen, sollten diejenigen Strategien entschlossen forciert werden, die geeignet sind, Kitas und Schulen prioritär offenzuhalten.

\section{Familienexpertise in politischen Entscheidungsgremien zur Pandemie verankern} In politischen Entscheidungsgremien zur Pandemie sollten Expertinnen und Experten der interdisziplinären Familienwissenschaft regelmäßig eingebunden werden. Da Maßnahmen wie Schulschließungen auch gesundheitliche Folgen implizieren, die sich im Pandemieverlauf kumulieren können, und eine große Zahl an Kindern und Jugendlichen davon betroffen ist, ist deren Situation schon rein aus gesundheitlicher Perspektive zu berücksichtigen. Dies sollte in den Abwägungsprozess zu Maßnahmen zur Eindämmung der Pandemie adäquat einfließen. Für die Abschätzung der Konsequenzen pandemiebekämpfender Maßnahmen auf die Lebenswirklichkeit, die Gesundheit, die Bildung - dabei insbesondere auf demografische Größenordnungen und soziale Ungleichheiten - ist entsprechende Expertise der Familienforschung notwendig.

\section{Lernrückstände langfristig aufholen, kurzfristig Lebensfreude und Freizeit} mit Gleichaltrigen ermöglichen

Angesichts der sich abzeichnenden Bildungsrückstände sollte beachtet werden, dass die Stärkung der Kinder und Jugendlichen in ihrem Selbstbewusstsein und ihrer psychischen Gesundheit mindestens ebenso wichtig ist. Kontakte mit Gleichaltrigen, Lebensfreude und altersgerechte Erlebnisse in Sport, Freizeit oder Reisen sind daher zukünftig zentral, nicht nur Nachhilfeangebote. Es gilt eine gute Balance zu finden, wonach Lernrückstände zunächst differenziert erfasst und dann langfristig aufgeholt werden sollten und kurz- und mittelfristig Lebensfreude und Freizeit mit Gleichaltrigen ermöglicht werden sollten. Denn psychisch gesunde und selbstsichere Kinder können mögliche Lernrückstände deutlich schneller und leichter aufholen. Schulen und bildungspolitische Entscheidungen müssen dies konsequent berücksichtigen. Hilfreich sind auch Programme, die geeignet sind, Kindern aus sozial schwachen Familien die Teilnahme an Ausflügen und Schulfahrten zu finanzieren. 


\section{Schulischen Druck reduzieren, belastete Schülerinnen und Schüler unterstützen}

Die Selbsteinschätzung einer depressiven Symptomatik ist nicht mit einer depressiven Erkrankung gleichzusetzen. Vielmehr sind die vorliegenden Daten, in denen $25 \%$ der 16- bis 19-Jährigen einen klinisch relevanten Schwellenwert erreichen, ein Hinweis auf eine belastete und gefährdete Gruppe von Jugendlichen, die teilweise an der Schwelle zu einer Depression stehen. Eine solche Entwicklung zeigt sich in Krisenzeiten, kann aber nach Beendigung der (externen) Krise sich in vielen Fällen wieder normalisieren. Ob sich eine depressive Symptomatik wirklich manifestiert, hängt auch von der Entwicklung der nächsten Monate ab. Dies betrifft den Verlauf der Pandemie, aber auch, welche Entscheidungen Politiker und Politikerinnen, Lehrkräfte und Bildungsfunktionäre und -funktionärinnen in den nächsten Monaten treffen und vor allem, wie Eltern mit der Situation umgehen. Um unnötigen Druck von den Kindern zu nehmen und für eine differenzierte Wahrnehmung psychisch beeinträchtigter Schülerinnen und Schüler (und ihre adäquate Unterstützung) bedarf es eines öffentlichen Diskurses und der Sensibilisierung von Schulleitungen sowie Lehrerinnen und Lehrern.

Wenn Kinder und Jugendliche wieder Zeit für Aktivitäten mit Gleichaltrigen bekommen und Lebensfreude zurückgewinnen und das schulische Aufholen ohne zu viel Druck und Verunsicherung gestaltet wird, wird sich auch die psychische Belastung der Jugendlichen verringern können. Wenn aber schulischer Druck auf die Kinder und Jugendlichen ausgeübt wird und sie Verunsicherung aufgrund der (pandemiebedingten) Lernrückstände erfahren, können sich depressive Symptomatiken in ernsthaften psychischen Erkrankungen, die nicht selten einen langfristigen Verlauf haben, manifestieren.

\section{Ausbau multidisziplinärer Schulsozialarbeit und schnellere Kassenzulassung für Psychotherapien}

Soziale und psychologische Dienstleistungen sollten für die Kinder und Jugendlichen, die gegenwärtig besonders gefährdet sind, ausreichend angeboten werden. Neben dem Ausbau der Schulsozialarbeit und der vermehrten Anstellung von Schulpsychologinnen und -psychologen müssen jedoch auch der ambulante und stationäre Sektor gestärkt werden. Neben Familientherapie und Paartherapie, die den familiären Rahmen der Kinder und Jugendlichen betreffen, benötigt es psychosoziale Beratung und Therapieplätze für die einzelnen Jugendlichen bzw. Kinder. Eine Möglichkeit, schnell zu reagieren, wäre z. B. Therapeutinnen und Therapeuten ohne Kassensitz eine Möglichkeit der Kassenabrechnung zu bieten oder mehr Kassenzulassungen zu ermöglichen. Damit könnten die Wartezeiten für psychotherapeutische Behandlungen verkürzt werden, und diese wären nicht mehr an die Möglichkeiten einzelner Familien gekoppelt, sich Sitzungen von Therapeutinnen und Therapeuten ohne Kassensitz leisten zu können. 


\section{Sprachförderung ausbauen, Kinder mit Migrationshintergrund zielgenau fördern}

Durch gezielte Sprachförderung sollten für Kinder, die zuhause nicht Deutsch sprechen, Auswirkungen von Schulschließungen in besonderer Weise aufgefangen werden. Generell sollte sprachliche Frühförderung bereits im Vorschulalter zügig ausgebaut werden, denn bereits vor der Pandemie zeichnete sich ein deutlicher Verbesserungsbedarf ab. Da im Kindesalter sogenannte Entwicklungsfenster für den Erwerb bestimmter Fähigkeiten existieren, vor allem auch für die sprachliche Entwicklung, sollten dafür möglichst schnell und umfassend standardisierte Test- und Förderprogramme angeboten werden, wie dies bereits im Corona-Aufholpaket der Bundesregierung angedacht ist und zielgruppenspezifische Förderung ermöglicht werden.

\section{Vereinssport und Schulsport stärken}

Sport, sowohl innerhalb der Bildungseinrichtungen als auch im organisierten Sport, bietet Kindern die Möglichkeit eines physisch und psychisch gesunden Aufwachsens und gleichzeitig auch die Möglichkeit, soziales Miteinander zu erlernen. Dafür sollten gerade Sportangebote für Kinder und Jugendliche so lange wie möglich auch bei einschränkenden Maßnahmen des Infektionsschutzes aufrechterhalten werden, ebenso Bewegungsangebote in Kitas und der Schulsport. Im Nachgang der Pandemie sollten vermehrt Bewegungsangebote für alle Kinder und Jugendlichen gefördert werden und z. B. neue Formen der Integration von Vereins- und Schulsport angedacht werden. Um den Vereinssport zu stärken und soziale Ungleichheiten zu reduzieren, ist es empfehlenswert, dass bei sozial schwächeren Familien die Vereinsbeiträge postpandemisch für einige Zeit übernommen werden.

\section{Leistungen der Eltern in der Pandemie anerkennen und Eltern zu Selbstsorge ermutigen}

Trotz oder gerade aufgrund hoher eigener Belastung bei beruflicher und zusätzlicher familialer Arbeit haben viele Eltern nur wenig Selbstsorge betrieben. Da viele sich über einen längeren Zeitraum primär Sorgen um ihre Kinder gemacht haben, sind nicht selten eigene Bedürfnisse und Wünsche hintangestellt worden. Für die Gesundheit und Leistungsfähigkeit dieser Eltern ist es wichtig, auch an eigene Bedarfe und die Selbstsorge zu denken. Hierzu sollten sie gesellschaftlich ermuntert werden und politische Unterstützung erhalten. Für besonders belastete Eltern sollten Mutter-Kind-Kuren oder Familienberatung möglich sein. Zudem muss Kinderförderung immer auch als Elternförderung gedacht werden. Kinder und Jugendliche reagieren unterschiedlich auf die Herausforderungen der Pandemie, d. h. sie sind unterschiedlich resilient. Ein wichtiger Faktor zur Stärkung der individuellen Resilienz ist das familiäre Umfeld. Je besser Eltern durch die Krise kommen, desto eher können Kinder in der Krisensituation Resilienz entwickeln. 


\section{Langfristiger Ausbau von Beratungs-, Bildungs- und Erholungsangeboten für Familien in Belastungssituationen}

Es bedarf eines langfristigen Programms, um diejenigen, die besonders belastet sind, nachhaltig zu unterstützen. Das Aufholpaket für 2021/2022 der Bundesregierung gibt hier bereits einige wichtige Impulse, da es mit seinen vier Säulen nicht nur schulisches Aufholen durch Nachhilfeprogramme und frühkindliche Bildung unterstützt. Durch kulturelle, sportliche und soziale Angebote soll einerseits das außerschulische Lernen gestärkt werden. Durch Ferienfreizeiten für Kinder oder Familienfreizeiten sollen andererseits auch Möglichkeiten zum „gemeinsamen Kraft tanken und Spaß haben für Familien“ geschaffen werden. Ein leichter Zugang besonders betroffener Familien, insbesondere solcher mit Migrationshintergrund, zu den Angeboten sollte gewährleistet werden. Auch bedarf es einer längerfristigen Verstetigung für Familien in Belastungssituationen. Um allerdings Kinder und Jugendliche im Alltag im Bedarfsfall adäquat und zeitnah unterstützen zu können, sollte das noch geringe Angebot an Schulsozialarbeit deutlich ausgebaut werden, ebenso Familienbildungs- und Familienberatungseinrichtungen. Diese Maßnahmen sollten auch über die unmittelbare Bewältigung der Pandemie hinaus Bestand haben.

\section{Endnoten}

\footnotetext{
${ }^{1}$ Die Daten entsprechen in der Größenordnung weitgehend denen von 2020 während der Corona-Krise.

${ }^{2}$ Das Kapitel 2 beruht in Teilen auf einer früheren BiB-Studie (Bujard et al. 2020b).

${ }^{3}$ Erster Lockdown: 36 \% Grundschülerinnen und -schüler, 30 \% Gymnasiasten, 34 \% Schülerinnen und Schüler anderer weiterführender Schulen; zweiter Lockdown: 42 \% Grundschülerinnen und -schüler, 33 \% Gymnasiasten, 26 \% Schülerinnen und Schüler anderer weiterführender Schulen (Wößmann et al. 2020b, 2021).

${ }^{4}$ Siehe Heuser 2021; Sina 2021; Davies 2021; UNICEF 2020, die sich aber auch auf die Jugend weltweit beziehen.

${ }^{5}$ Dies gilt für die COVIDiSTRESS-Studie (Kowal et al. 2020), die Studie „Familien und Kitas“ (Cohen et al. 2020), die Studien von Meyer et al. (2021; 2020), die KiCo-Studie (Andresen et al. 2020b), die WZB-Studie „Corona-Alltag“ (Bünning et al. 2020), die PACO-Studie (Schmidt et al. 2021), die Studie um das Autorenteam Czymara, Cano und Langenkamp (Czymara et al. 2020; Langenkamp et al. 2020) sowie die Kindernetzwerkstudie (Langer und Barth 2020).

${ }^{6}$ Andere Bereiche, die ebenfalls mit Blick auf Belastungen für Eltern untersucht wurden, sind z. B. die finanzielle Situation (Andresen et al. 2020b; Bünning et al. 2020; BMFSFJ 2020), die Arbeitsplatzsicherheit (Bünning et al. 2020; BMFSFJ 2020), die Gesundheit der Familie (Cohen et al. 2020) oder Streit, Spannungen und Gewalt zu Hause (BMFSFJ 2020; Technische Universität München 2020; Forsa 2020c).
} 


\section{Literatur}

Allmendinger, Jutta (2020): Zurück in alte Rollen. Corona bedroht die Geschlechtergerechtigkeit. In: WZB Mitteilungen (168), S. 45-47, zuletzt geprüft am 21.06.2021.

Andresen, Sabine; Heyer, Lea; Lips, Anna; Rusack, Tanja; Schröer, Wolfgang; Thomas, Severine; Wilmes, Johanna (2020a): „Die Corona-Pandemie hat mir wertvolle Zeit genommen". Jugendalltag 2020. Universitätsverlag Hildesheim. Hildesheim.

Andresen, Sabine; Lips, Anna; Möller, Renate; Rusack, Tanja; Schröer, Wolfgang; Thomas, Severine; Wilmes, Johanna (2020b): Kinder, Eltern und ihre Erfahrungen während der Corona-Pandemie. Erste Ergebnisse der bundesweiten Studie KiCo. Universitätsverlag Hildesheim. Hildesheim.

Anger, Silke; Bernhard, Sarah; Dietrich, Hans; Lerche, Adrian; Patzina, Alexander; Sandner, Malte; Toussaint, Carina (2021): Der Abiturjahrgang 2021 in Zeiten von Corona: Zukunftssorgen und psychische Belastungen nehmen zu. Institut für Arbeitsmarkt und Berufsforschung (IAB). Nürnberg (IAB-Forum). Online verfügbar unter https://www.iab-forum.de/der-abiturjahrgang-2021-in-zeiten-von-corona-zukunftssorgen-und-psychische-belastungen-nehmen-zu/.

Anger, Silke; Sandner, Malte (2020): Die Auswirkungen der Coronakrise auf die Arbeitsmarktchancen der Corona-Abiturjahrgänge. In: ifo-Schnelldienst 73 (9), S. 3-7.

Baker, Michael (2013): Industrial actions in schools: Strikes and student achievement. In: Canadian Journal of Economics 46 (3), S. 1014-1036.

Barlovic, Ingo; Ullrich, Denise; Wieland, Clemens (2020): Ausbildungsperspektiven in Zeiten von Corona. Eine repräsentative Befragung von Jugendlichen. Gütersloh: Bertelsmann Stiftung.

Barlovic, Ingo; Ullrich, Denise; Wieland, Clemens (2021): Ausbildungsperspektiven im zweiten Corona-Jahr. Eine repräsentative Befragung von Jugendlichen 2021. Gütersloh: Bertelsmann Stiftung.

Baumann, Menno; Berghäuser, Andree; Bolz, Tijs; Martens, Thomas (2021): Den Fokus neu denken - Skizze eines Pandemiemanagements auf Grundlage der Bedürfnisse und Lern- und Entwicklungserfordernissen von Kindern, Jugendlichen und Familien (Socialnet Discussion Paper).

Bayer Vital (2021): Bepanthen-Kinderförderung veröffentlicht forsa-Umfrage zu aktuellem Stimmungsbild in Familien: Trotz Krise - Mehrheit der Eltern blickt optimistisch in die Zukunft. Leverkusen, zuletzt geprüft am 15.02.2021.

Belot, Michèle; Webbink, Dinand (2010): Do Teacher Strikes Harm Educational Attainment of Students? In: Labour 24 (4), S. 391-406. DOI: 10.1111/j.1467-9914.2010.00494.x.

Bignardi, Giacomo; Dalmaijer, Edwin S.; Anwyl-Irvine, Alexander L.; Smith, Tess A.; Siugzdaite, Roma; Uh, Stepheni; Astle, Duncan E. (2020): Longitudinal increases in childhood depression symptoms during the COVID-19 lockdown. In: Archives of disease in childhood. DOI: 10.1136/archdischild-2020-320372.

BJK (2021): Kindheit und Jugend in Zeiten von Corona. Konsequenzen für die aktuelle und zukünftige Kinder- und Jugendpolitik. Hg. v. Bundesjugendkuratorium (BJK). München.

BMBF; BMFSFJ (2021): Aktionsprogramm Aufholen nach Corona für Kinder und Jugendliche für die Jahre 2021 und $2022-2$ Mrd. Euro. Hg. v. Bundesministerium für Bildung und Forschung und Bundesministerium für Familie, Senioren, Frauen und Jugend.

BMFSFJ (2020): Familien in der Corona-Zeit: Herausforderungen, Erfahrungen und Bedarfe. Ergebnisse einer repräsentativen Elternbefragung im April und Mai 2020. Hg. v. BMFSFJ. Berlin.

Brazendale, Keith; Beets, Michael W.; Weaver, R. Glenn; Pate, Russell R.; Turner-McGrievy, Gabrielle M.; Kaczynski, Andrew T. et al. (2017): Understanding differences between summer vs. school obesogenic behaviors of children. The structured days hypothesis. In: The international journal of behavioral nutrition and physical activity 14 (1), S. 100. DOI: 10.1186/s12966-017-0555-2.

Brüderl, Josef; Drobnič, Sonja; Hank, Karsten; Neyer, Franz J.; Walper, Sabine; Alt, Philipp et al. (2020): The German Family Panel (pairfam). GESIS Data Archive, Cologne. ZA5678 Data file Version 11.0.0. 
Bühring, Petra (2020): Onlinespiele und soziale Medien: Corona verstärkt die Sucht. In: Deutsches Aerzteblatt (8), S. 337.

Bühring, Petra (2021): Coronapandemie: „Die Situation der Kinder spitzt sich jetzt dramatisch zu“. In: Deutsches Aerzteblatt (4), S. 151.

Bujard, Martin; Diehl, Claudia; Kreyenfeld, Michaela; Leyendecker; Birgit; Spieß; C. Katharina (2020a): Geflüchtete, Familien und ihre Kinder. Warum der Blick auf die Familien und die Kindertagesbetreuung entscheidend ist. In: Sozialer Fortschritt 69 (8-9), S. 561-577. Online verfügbar unter Doi: 10.3790/sfo.69.8-9.561.

Bujard, Martin; Laß, Inga; Diabaté, Sabine; Sulak, Harun; Schneider, Norbert F. (2020b): Eltern während der Corona-Krise. Zur Improvisation gezwungen. In: BiB.Bevölkerungs. Studien (1/2020). DOI: 10.12765/bro-2020-01.

Bundesgesundheitsministerium (2021): Coronavirus-Pandemie (SARS-CoV-2): Chronik bisheriger Maßnahmen und Ereignisse. Online verfügbar unter https://www.bundesgesundheitsministerium.de/coronavirus/chronik-coronavirus.html, zuletzt geprüft am 02.07.2021.

Bundesregierung (2021): Das sind die geltenden Regeln und Einschränkungen. Online verfügbar unter https://www. bundesregierung.de/breg-de/themen/coronavirus/corona-diese-regeln-und-einschraenkung-gelten-1734724, zuletzt geprüft am 02.07.2021.

Bünning, Mareike; Hipp, Lena; Munnes, Stefan (2020): Erwerbsarbeit in Zeiten von Corona. WZB Ergebnisbericht. Hg. v. Wissenschaftszentrum Berlin für Sozialforschung. Berlin.

Calvano, Claudia; Engelke, Lara; Di Bella, Jessica; Kindermann, Jana; Renneberg, Babette; Winter, Sibylle M. (2021): Families in the COVID-19 pandemic. Parental stress, parent mental health and the occurrence of adverse childhood experiences-results of a representative survey in Germany. In: European child \& adolescent psychiatry. DOI: 10.1007/ s00787-021-01739-0.

Clemens, Vera; Köhler-Dauner, Franziska; Keller, Ferdinand; Ziegenhain, Ute; Fegert, Jörg M.; Kölch, Michael (2021): Gewalt in intimen Partnerschaften und psychische Probleme bei Kindern und Jugendlichen. Online-Survey während der COVID-19-Pandemie. In: Psychotherapeut 66, S. 209-216. DOI: 10.1007/s00278-021-00501-w.

Cohen, Franziska; Oppermann, Elisa; Anders, Yvonne (2020): Familien und Kitas in der Corona-Zeit. Zusammenfassung der Ergebnisse. Hg. v. Otto-Friedrich-Universität Bamberg. Bamberg.

Crescentini, Cristiano; Feruglio, Susanna; Matiz, Alessio; Paschetto, Andrea; Vidal, Enrico; Cogo, Paola; Fabbro, Franco (2020): Stuck Outside and Inside. An Exploratory Study on the Effects of the COVID-19 Outbreak on Italian Parents and Children's Internalizing Symptoms. In: Frontiers in psychology 11, S. 586074. DOI: 10.3389/fpsyg.2020.586074.

Czymara, Christian S.; Langenkamp, Alexander; Cano, Tomás (2020): Cause for concerns. Gender inequality in experiencing the COVID-19 lockdown in Germany. In: European Societies 159 (3), S. 1-14. DOI: 10.1080/14616696.2020.1808692.

Danzer, Natalia; Huebener, Mathias; Pape, Astrid; Spiess, C. Katharina; Siegel, Nico A.; Wagner, Gert G. (2021): Cracking under Pressure? Gender Role Attitudes toward Maternal Employment in Times of a Pandemic. Hg. v. Institute of Labor Economics. Bonn (IZA Discussion Paper, 14471).

Das deutsche Schulportal (2021): „Wir werden zusätzliches Personal in die Schulen holen“. Ein Jahr Corona-Pandemie. Interview mit Kultusministerkonferenz-Präsidentin Britta Ernst. https://deutsches-schulportal.de/bildungswesen/ britta-ernst-kultusministerkonferenz-wir-werden-zusaetzliches-personal-in-die-schulen-holen/.

Davies, Claudia (2021): Verlorene Generation? Zerstört die Corona-Pandemie die Zukunft der Jugend? Phoenix Plus, 2021, zuletzt geprüft am 01.07.2021.

Depping, Denise; Lücken, Markus; Musekamp, Frank; Thonke, Franziska (2021): Kompetenzstände Hamburger Schüler*innen vor und während der Corona-Pandemie. In: Detlef Fickermann, Benjamin Edelstein, Manja Attig, Sonja Blum, Denise Depping, Ivana Dobrotic et al. (Hg.): Schule während der Corona-Pandemie. Neue Ergebnisse und Überblick über ein dynamisches Forschungsfeld. 1. Auflage. Münster: Waxmann (Die Deutsche Schule. Zeitschrift für Erziehungswissenschaft, Bildungspolitik und pädagogische Praxis - Beiheft, 17), S. 51-79.

Deutsche Gesellschaft für Kinder- und Jugendpsychiatrie, Psychosomatik und Psychotherapie (2021): Unwahre Behauptungen über „Triage“ in der Kinder- und Jugendpsychiatrie. 19.05.2021. Online verfügbar unter https://www. dgkjp.de/unwahre-behauptungen-ueber-triage-in-der-kinder-und-jugendpsychiatrie/. 
Deutsche Lebens-Rettungs-Gesellschaft (2017): Schwimmfähigkeit der Bevölkerung. Ergebnisse einer repräsentativen Bevölkerungsumfrage.

Deutscher Olympischer Sportbund (Hg.) (2021): Bestandserhebung 2020. Frankfurt a. M. Online verfügbar unter https://www.dosb.de/medienservice/statistiken.

Deutsches Ärzteblatt (2021): Kinder und Jugendliche suchen vermehrt Hilfe bei Psychotherapeuten. In: Deutsches Ärzteblatt, 2021 (19.02.2021). Online verfügbar unter https://www.aerzteblatt.de/nachrichten/121321/Kinder-und-Jugendliche-suchen-vermehrt-Hilfe-bei-Psychotherapeuten.

Dietrich, Hans; Patzina, Alexander; Lerche, Adrian (2021): Social inequality in the homeschooling efforts of German high school students during a school closing period. In: European Societies 23 (sup1), S348-S369. DOI: $10.1080 / 14616696.2020 .1826556$.

DIW; Infratest dimap (2021): FamilienMonitor_Corona. Interaktive Grafiken von Infratest dimap. Online verfügbar unter https://www.diw.de/de/diw_01.c.809410.de/familienmonitor_corona.html, zuletzt geprüft am 11.02.2021.

dpa (2021): So sehr kann die Pandemie Jugendliche krank machen. In: dpa 2021, 16.02.2021. Online verfügbar unter https://www.msn.com/de-de/gesundheit/medizinisch/so-sehr-kann-die-pandemie-jugendliche-krank-machen/ ar-BB1dIV9M.

Engzell, Per; Frey, Arun; Verhagen, Mark D. (2021): Learning Loss Due to School Closures During the COVID-19 Pandemic. In: SocArXiv.

Fegert, Jörg M.; Berthold, Oliver; Clemens, Vera; Kölch, Michael (2020): COVID-19-Pandemie: Kinderschutz ist systemrelevant. In: Deutsches Aerzteblatt Online 117 (14).

Fegert, Jörg M.; Kehoe, Laura A.; Çuhadaroglu Çetin, Fusun; Doyle, Maeve; Eliez, Stephan; Hebebrand, Johannes et al. (2021): Next generation Europe. A recovery plan for children, adolescents and their families. In: European child $\&$ adolescent psychiatry. Online verfügbar unter https://doi.org/10.1007/s00787-021-01767-w.

Forsa (2020a): Homeschooling in Corona-Zeiten. Erfahrungen von Eltern und Schülern. Ergebnisse einer Eltern-Kind-Befragung mit forsa.omninet. Berlin.

Forsa (2020b): Das Deutsche Schulbarometer Spezial Corona Krise. Ergebnisse einer Befragung von Lehrerinnen und Lehrern an allgemeinbildenden Schulen im Auftrag der Robert Bosch Stiftung in Kooperation mit der ZEIT.

Forsa (2020c): Das Deutsche Schulbarometer Spezial Corona-Krise: Folgebefragung. Ergebnisse einer Befragung von Lehrerinnen und Lehrern an allgemeinbildenden Schulen im Auftrag der Robert Bosch Stiftung in Kooperation mit der ZEIT. Hg. v. Forsa.

Fuchs-Schündeln, Nicola; Stephan, Gesine (2020): Bei drei Vierteln der erwerbstätigen Eltern ist die Belastung durch Kinderbetreuung in der Covid-19-Pandemie gestiegen. Online verfügbar unter https://www.iab-forum.de/bei-dreivierteln-der-erwerbstaetigen-eltern-ist-die-belastung-durch, zuletzt geprüft am 10.02.2021.

Guan, Hongyan; Okely, Anthony D.; Aguilar-Farias, Nicolas; del Pozo Cruz, Borja; Draper, Catherine E.; El Hamdouchi, Asmaa et al. (2020): Promoting healthy movement behaviours among children during the COVID-19 pandemic. In: The Lancet Child \& Adolescent Health 4 (6), S. 416-418. DOI: 10.1016/S2352-4642(20)30131-0.

Hammerstein, Svenja; König, Christoph; Dreisoerner, Thomas; Frey, Andreas (2021): Effects of COVID-19-Related School Closures on Student Achievement. A Systematic Review.

Hank, Karsten; Steinbach, Anja (2021): The virus changed everything, didn't it? Couples' division of housework and childcare before and during the Corona crisis. In: Journal of Family Research 33 (1), S. 99-114. DOI: 10.20377/jfr-488.

Heuser, Olaf (2021): Die verlorene Lockdown-Generation (Acht Milliarden - Der Auslandspodcast). Der Spiegel, 26.02.2021. Online verfügbar unter https://www.spiegel.de/ausland/corona-pandemie-die-verlorene-lockdowngeneration-podcast-a-43b18589-cb8f-47e9-9932-80e23fcd3567.

Hövermann, Andreas (2021): Belastungswahrnehmung in der Corona-Pandemie. Erkenntnisse aus vier Wellen der HBS-Erwerbspersonenbefragung 2020/21. Hg. v. Hans-Böckler-Stiftung. Düsseldorf.

Huber, Stephan Gerhard; Günther, Paula Sophie; Schneider, Nadine; Helm, Christoph; Schwander, Marius; Schneider, Julia; Pruitt, Jane (2020): COVID-19 und aktuelle Herausforderungen in Schule und Bildung: Waxmann Verlag GmbH. 
Huber, Stephan Gerhard; Helm, Christoph (2020): Lernen in Zeiten der Corona-Pandemie. Die Rolle familiärer Merkmale für das Lernen von Schüler*innen: Befunde vom Schul-Barometer in Deutschland, Österreich und der Schweiz. In: DDS - Die Deutsche Schule, S. 37-60. DOI: 10.31244/9783830992318.02.

Huebener, Mathias; Siegel, Nico A.; Spieß, C. Katharina; Spinner, Christian; Wagner, Gert G. (2021): Kein „Entweder-oder": Eltern sorgen sich im Lockdown um Bildung und Gesundheit ihrer Kinder. DIW. Berlin (DIW aktuell).

Huebener, Mathias; Spieß, C. Katharina; Siegel, Nico A.; Wagner, Gert G. (2020): Wohlbefinden von Familien in Zeiten von Corona. Eltern mit jungen Kindern am stärksten beeinträchtigt. Hg. v. Deutsches Institut für Wirtschaftsforschung (DIW). Berlin (DIW Wochenbericht, 30+31), zuletzt geprüft am 11.02.2021.

Huxhold, Oliver; Tesch-Römer, Clemens (2021): Einsamkeit steigt in der Corona-Pandemie bei Menschen im mittleren und hohen Erwachsenenalter gleichermaßen deutlich. Deutsches Zentrum für Altersfragen (DZA aktuell, 4/2021). Online verfügbar unter https://www.bmfsfj.de/blob/jump/173820/einsamkeit-deutscher-alterssurvey-dzi-data.pdf.

International Union for Health Promotion and Education (2021): Critical Actions for Mental Health Promotion. Position statement of the International Union for Health Promotion and Education. Hg. v. International Union for Health Promotion and Education. International Union for Health Promotion and Education. Online verfügbar unter https://www.iuhpe.org/index.php/en/iuhpe-at-a-glance/iuhpe-official-statements.

Jaume, David; Willén, Alexander (2019): The long-run effects of teacher strikes: Evidence from Argentina. In: Journal of Labor Economics 37 (4), S. 1097-1139.

Jessen, Jonas; Spieß, C. Katharina; Wrohlich, Katharina (2021): Sorgearbeit während der Corona-Pandemie. Mütter übernehmen größeren Anteil - vor allem bei schon zuvor ungleicher Aufteilung. In: DIW Wochenbericht (9), S. 132-139.

Juncke, David; Lehmann, Klaudia; Nicodemus, Johanna; Stoll, Evelyn; Weuthen, Ulrich (2021): Familienbildung und Familienberatung in Deutschland. Eine Bestandsaufnahme. Prognos AG. Düsseldorf. Online verfügbar unter https:// www.bildungsserver.de/fisaktuell.html?FIS_akt_Nr=38481.

Karlsruher Institut für Technologie (11.12.2020): Kaum Sport - aber mehr Bewegung im Lockdown. Online verfügbar unter https://idw-online.de/de/news759834.

Kauer-Berk, Oliver; Burrmann, Ulrike; Derecik, Ahmet; Gieß-Stüber, Petra; Kuhlmann, Detlef; Neuber, Nils et al. (2020): Das Virus, der Sport und die Herausforderungen. In: Forum Kind Jugend Sport 1 (2), S. 100-109. DOI: 10.1007/ s43594-020-00016-3.

Klasen, F.; Meyrose, A.-K.; Otto, C.; Reiss, F.; Ravens-Sieberer, U. (2017): Psychische Auffälligkeiten von Kindern und Jugendlichen in Deutschland. In: Monatsschr Kinderheilkd 165 (5), S. 402-407. DOI: 10.1007/s00112-017-0270-8.

Knolle, Franziska; Ronan, Lisa; Murray, Graham K. (2021): The impact of the COVID-19 pandemic on mental health in the general population. A comparison between Germany and the UK. In: BMC psychology 9 (1), S. 60. DOI: 10.1186/ s40359-021-00565-y.

Kohlrausch, Bettina; Zucco, Aline (2020): Die Corona-Krise trifft Frauen doppelt. Weniger Erwerbseinkommen und mehr Sorgearbeit. Düsseldorf (Policy Brief WSI, 40), zuletzt geprüft am 28.06.2021.

Koletzko, Berthold; Holzapfel, Christina; Schneider, Ulrike; Hauner, Hans (2021): Lifestyle and Body Weight Consequences of the COVID-19 Pandemic in Children. Increasing Disparity. In: Annals of nutrition \& metabolism, S. 1-3. DOI: $10.1159 / 000514186$.

Kowal, Marta; Coll-Martín, Tao; Ikizer, Gözde; Rasmussen, Jesper; Eichel, Kristina; Studzińska, Anna et al. (2020): Who is the Most Stressed During the COVID-19 Pandemic? Data from 26 Countries and Areas. In: Applied psychology. Health and well-being 12 (4), S. 946-966. DOI: 10.1111/aphw.12234.

Kreyenfeld, Michaela; Bujard, Martin (2021): Wenn Eltern überfordert werden. In: DIW Berlin: Politikberatung kompakt (163), S. 36-41.

Kreyenfeld, Michaela; Zinn, Sabine (2021): Coronavirus and care. How the coronavirus crisis affected fathers' involvement in Germany. In: Demographic Research 44, S. 99-124. DOI: 10.4054/DemRes.2021.44.4.

Kugelmeier, Dorothea; Schmolze-Krahn, Raimund (2020a): Nach dem Frühjahrs-Lockdown: Entspannung in der Corona-Lage bleibt aus. So erlebten beeinträchtigte Kinder und ihre Eltern den Pandemie-Sommer. Fraunhofer-Institut für angewandte Informationstechnik; inclusion tech lab. 
Kugelmeier, Dorothea; Schmolze-Krahn, Raimund (2020b): Schulöffnungen: Ein Tropfen auf den heissen Stein. So leiden beeinträchtigte Kinder und ihre Eltern unter der Corona-Krise. Fraunhofer-Institut für angewandte Informationstechnik; inclusion tech lab.

Kultusministerkonferenz (2017): Empfehlungen der Ständigen Konferenz der Kultusminister der Länder in der Bundesrepublik Deutschland, der Deutschen Vereinigung für Sportwissenschaft und des Bundesverbandes zur Förderung der Schwimmausbildung für den Schwimmunterricht in der Schule.

Langenkamp, Alexander; Cano, Tomas; Czymara, Christian S. (2020): Family structure and concerns during the COVID-19 pandemic. A computational, mixed-method approach. In: preprint. DOI: 10.31235/osf.io/6c42q.

Langer, Thorsten; Barth, Michael (2020): Erste Ergebnisse der Covid-19 Kindernetzwerk-Studie auf einen Blick. Hg. v. Kindernetzwerk e.V. Online verfügbar unter https://www.kindernetzwerk.de/de/agenda/News/2020/1207Erste-Ergebnisse-auf-einen-Blick.php, zuletzt geprüft am 15.02.2021.

Langmeyer, Alexandra; Guglhör-Rudan, Angelika; Naab, Thorsten; Urlen, Marc; Winklhofer, Ursula (2020): Kind sein in Zeiten von Corona. Ergebnisbericht zur Situation von Kindern während des Lockdowns im Frühjahr 2020. DJI Deutsches Jugendinstitut. München.

Lee, Shawna J.; Ward, Kaitlin P.; Lee, Joyce Y.; Rodriguez, Christina M. (2021): Parental Social Isolation and Child Maltreatment Risk during the COVID-19 Pandemic. In: Journal of Family Violence. DOI: 10.1007/s10896-020-00244-3.

Lehr, Dirk; Hillert, Andreas; Schmitz, Edgar; Sosnowsky, Nadia (2008): Screening depressiver Störungen mittels Allgemeiner Depressions-Skala (ADS-K) und State-Trait Depressions Scales (STDS-T). Eine vergleichende Evaluation von Cut-Off-Werten. In: Diagnostica 54 (1), S. 61-70. DOI: 10.1026/0012-1924.54.2.61.

Lemm, Saskia (2021): COPSY-Studie: Kinder und Jugendliche leiden psychisch weiterhin stark unter Corona-Pandemie. Online verfügbar unter https://www.uke.de/kliniken-institute/kliniken/kinder-und-jugendpsychiatrie-psychotherapie-und-psychosomatik/forschung/arbeitsgruppen/child-public-health/forschung/copsy-studie.html.

Leopoldina (2021): Kinder und Jugendliche in der Coronavirus-Pandemie: psychosoziale und edukative Herausforderungen und Chancen. 8. Ad-hoc-Stellungnahme. Hg. v. Leopoldina - Nationale Akademie der Wissenschaften. Online verfügbar unter https://www.leopoldina.org/publikationen/detailansicht/publication/kinder-und-jugendliche-in-der-coronavirus-pandemie-psychosoziale-und-edukative-herausforderungen-un/, zuletzt geprüft am 01.07.2021.

Li, Xiaowei; Zhou, Siyu (2021): Parental worry, family-based disaster education and children's internalizing and externalizing problems during the COVID-19 pandemic. In: Psychological trauma: theory, research, practice and policy. DOI: 10.1037/tra0000932.

Lockl, Kathrin; Attig, Manja; Nusser, Lena; Wolter, Ilka (2021): Lernen im Lockdown: Welche Voraussetzungen helfen Schülerinnen und Schülern? Die Bedeutsamkeit der Lesekompetenz, des Interesses an Lerninhalten und der Anstrengungsbereitschaft für die Bewältigung des Lernens zuhause. Leibniz-Institut für Bildungsverläufe e.V. Bamberg (NEPS Corona \& Bildung, 5).

Maier, Tobias (2020): Auswirkungen der „Corona-Krise“ auf die duale Berufsausbildung: Risiken, Konsequenzen und Handlungsnotwendigkeiten. Hg. v. Bundesinstitut für Berufsbildung (BIBB) (BIBB Preprint). Online verfügbar unter urn:nbn:de:0035-vetrepository-776778-8.

Mairhofer, Andreas; Peucker, Christian; Pluto, Liane; van Santen, Eric; Seckinger, Mike (2020): Kinder- und Jugendhilfe in Zeiten der Corona-Pandemie. DJI-Jugendhilfeb@rometer bei Jugendämtern. Unter Mitarbeit von Monika Gandlgruber. München: Deutsches Jugendinstitut.

Meyer, Bertolt; Zill, Alexander; Dilba, Dominik; Gerlach, Rebecca; Schumann, Susen (2021): Employee psychological well-being during the COVID-19 pandemic in Germany. A longitudinal study of demands, resources, and exhaustion. In: International journal of psychology : Journal international de psychologie. DOI: 10.1002/ijop.12743.

Meyer, Bertolt; Zill, Alexander; Schumann, Susen (2020): Arbeitssituation und Belastung zu Zeiten der Corona-Pandemie. In: Techniker Krankenkasse (Hg.): Corona 2020. Gesundheit, Belastungen, Möglichkeiten, S. 46-67, zuletzt geprüft am 11.02.2021.

Möhring, Katja; Naumann, Elias; Reifenscheid, Maximiliane; Blom, Annelies G.; Wenz, Alexander; Rettig, Tobias et al. (2020): Die Mannheimer Corona-Studie. Schwerpunktbericht zu Erwerbstätigkeit und Kinderbetreuung. Hg. v. Universität Mannheim. Mannheim. 
Moore, Sarah A.; Faulkner, Guy; Rhodes, Ryan E.; Brussoni, Mariana; Chulak-Bozzer, Tala; Ferguson, Leah J. et al. (2020): Impact of the COVID-19 virus outbreak on movement and play behaviours of Canadian children and youth. A national survey. In: The international journal of behavioral nutrition and physical activity 17 (1), S. 85. DOI: 10.1186/ s12966-020-00987-8.

Müller, Kai-Uwe; Samtleben, Claire; Schmieder, Julia; Wrohlich, Katharina (2020): Corona-Krise erschwert Vereinbarkeit von Beruf und Familie vor allem für Mütter - Erwerbstätige Eltern sollten entlastet werden. In: DIW Wochenbericht (19), S. 331-340, zuletzt geprüft am 15.02.2021.

Oerter, Rolf; Montada, Leo (Hg.) (2008): Entwicklungspsychologie. 6. Aufl. Weinheim: Beltz. Online verfügbar unter http://www.content-select.com/index.php?id=bib_view\&ean=9783621278478.

Polanczyk, Guilherme V.; Salum, Giovanni A.; Sugaya, Luisa S.; Caye, Arthur; Rohde, Luis A. (2015): Annual research review. A meta-analysis of the worldwide prevalence of mental disorders in children and adolescents. In: Journal of child psychology and psychiatry, and allied disciplines 56 (3), S. 345-365. DOI: 10.1111/jcpp.12381.

Provona BKK (2020): Psychische Gesundheit in der Krise. Ergebnisse einer Befragung unter Psychiatern und Psychotherapeuten. Hg. v. Provona BKK. Leverkusen, zuletzt geprüft am 11.02.2021.

Rathgeb, Thomas (2020): JIMplus 2020. Lernen und Freizeit in der Corona-Krise. Hg. v. Medienpädagogischer Forschungsverbund Südwest. Stuttgart.

Ravens-Sieberer, Ulrike; Kaman, Anne; Erhart, Michael; Devine, Janine; Schlack, Robert; Otto, Christiane (2021): Impact of the COVID-19 pandemic on quality of life and mental health in children and adolescents in Germany. In: European child \& adolescent psychiatry. DOI: 10.1007/s00787-021-01726-5.

Ravens-Sieberer, Ulrike; Otto, Christiane; Kaman, Anne; Adedeji, Adekunle; Devine, Janine; Napp, Ann-Kathrin et al. (2020): Mental health and quality of life in children and adolescents during the COVID-19 pandemic. In: Deutsches Aerzteblatt Online. DOI: 10.3238/arztebl.2020.0828.

Rebar, Amanda L.; Stanton, Robert; Geard, David: Short, Camille; Duncan, Mitch J.; Vandelanotte, Corneel A. (2015): A meta-meta-analysis of the effect of physical activity on depression and anxiety in non-clinical adult populations. In: Health Psychology Review 9 (3), S. 366-378. DOI: 10.1080/17437199.2015.1022901.

Rieger, Maximilian (2021): So geht es den Sportvereinen nach einem Jahr Lockdown. In: Deutschlandfunk, 21.03.2021. Online verfügbar unter https://www.deutschlandfunk.de/corona-und-breitensport-so-geht-es-den-sportvereinennach.1346.de.html?dram:article_id=494486.

Ringler, Paul; Baumegger, David (2020): Zur Situation von Eltern während des zweiten Lockdowns in der Coronapandemie. Wien.

RKI (2021): COVID-19: Fallzahlen in Deutschland und weltweit. Hg. v. Robert Koch-Institut. Online verfügbar unter https://www.rki.de/DE/Content/InfAZ/N/Neuartiges_Coronavirus/Fallzahlen.html, zuletzt geprüft am 20.06.2021.

Schlack, Robert; Neuperdt, Laura; Hölling, Heike; Bock, Freia de; Ravens-Sieberer, Ulrike; Mauz, Elvira et al. (2020): Auswirkungen der COVID-19-Pandemie und der Eindämmungsmaßnahmen auf die psychische Gesundheit von Kindern und Jugendlichen. In: Journal of Health Monitoring 5 (4). DOI: 10.25646/7173.

Schmid, Raimund (2020): Wie Corona auf Jugendliche wirkt. Kontaktsperren und weniger Bewegung: Die Corona-Pandemie belastet Jugendliche mit Vorerkrankungen enorm, mahnt Jugendforscher Klaus Hurrelmann. Aber auch die gesunden unter innen leiden - psychisch und physisch. In: ÄrzteZeitung, 2020. Online verfügbar unter https://www.aerztezeitung.de/Politik/Wie-Corona-auf-Jugendliche-wirkt-409712.html.

Schmidt, Andrea; Kramer, Andrea C.; Brose, Annette; Schmiedeck, Florian; Neubauer, Andreas B. (2021): Homeschooling and affective well-being of parents and children during the COVID-19 pandemic. A daily diary study. In: unveröffentlichtes Manuscript, zuletzt geprüft am 12.02.2021.

Schmidt, Steffen C. E.; Anedda, Bastian; Burchartz, Alexander; Eichsteller, Ana; Kolb, Simon; Nigg, Carina et al. (2020): Physical activity and screen time of children and adolescents before and during the COVID-19 lockdown in Germany. A natural experiment. In: Scientific reports 10 (1), S. 21780. DOI: 10.1038/s41598-020-78438-4.

Schmiedeberg, Claudia; Thönnissen, Carolin (2021): Positive and negative perceptions of the COVID-19 pandemic: Does personality play a role? In: Social Science \& Medicine 276. DOI: 10.1016/j.socscimed.2021.113859. 
Schneider, Ulrike; Hauner, Hans; Klingenspor, Martin; Witt, Heiko; Koletzko, Berthold (2020): Lebensstil und Ernährung in Corona-Zeiten. Pressekonferenz/Expertengespräch zur Forsa-Studie, 2020.

Schneider, William; Waldfogel, Jane; Brooks-Gunn, Jeanne (2017): The Great Recession and risk for child abuse and neglect. In: Children and youth services review 72, S. 71-81. DOI: 10.1016/j.childyouth.2016.10.016.

Schober, Pia; Stahl, Juliane (2014): Childcare trends in Germany. Increasing socio-economic disparities in East and West. In: DIW Economic Bulletin 4 (11), 51-58, zuletzt geprüft am 11.02.2021.

Schult, Johannes; Mahler, Nicole; Fauth, Benjamin; Lindner, Marlit A. (2021): Did Students Learn Less During the COVID-19 Pandemic? Reading and Mathematics Competencies Before and After the First Pandemic Wave. PsyArXiv.

Sina, Marie (2021): Junge Erwachsene in der Coronakrise - Europas verlorene Corona-Generation? DW Deutsche Welle, 03.04.2021. Online verfügbar unter https://www.dw.com/de/europas-verlorene-corona-generation/a-57045582.

Spaderna, Heike; Schmukle, Stefan C.; Krohne; Heinz Walter (2002): Bericht über die deutsche Adaptation der State-Trait Depression Scales (STDS). In: Diagnostica 48 (2), S. 80-89. DOI: 10.1026//0012-1924.48.2.80.

Statistisches Bundesamt (2020): Bildung und Kultur - Allgemeinbildende Schulen. Schuljahr 2019/2020.

Statistisches Bundesamt (2021): Bevölkerung und Erwerbstätigkeit 2019. Bevölkerungsfortschreibung auf Grundlage des Zensus 2011. Hg. v. Statistisches Bundesamt (Destatis) (Fachserie 1 Reihe 1.3). Online verfügbar unter https:// www.destatis.de/DE/Service/Bibliothek/_publikationen-fachserienliste-1.html?nn=206136.

Statistisches Bundesamt (2021b): 9 \% mehr Fälle: Jugendämter melden 2020 Höchststand an Kindeswohlgefährdungen. Wiesbaden.

StäwiKo (2021): Pandemiebedingte Lernüuckstände aufholen - Unterstützungsmaßnahmen fokussieren, verknüpfen und evaluieren. Hg. v. Ständige wissenschaftliche Kommission der KMK. Online verfügbar unter https://www. ipn.uni-kiel.de/de/das-ipn/nachrichten/StawiKoStellungnahme_PandemiebedingteLernruckstandeaufholen.pdf.

Technische Universität München (2020): Häusliche Gewalt während der Corona- Pandemie. Online verfügbar unter https://www.tum.de/studinews/ausgabe-032015/show-032015/article/36053/, zuletzt geprüft am 11.02.2021.

Timmons, Brian W.; LeBlanc, Allana G.; Carson, Valerie; Connor Gorber, Sarah; Dillman, Carrie; Janssen, Ian et al. (2012): Systematic review of physical activity and health in the early years (aged 0-4 years). In: Applied Physiology, Nutrition, and Metabolism 37 (4), S. 773-792. DOI: 10.1139/h2012-070.

Tomasik, Martin J.; Helbling, Laura A.; Moser, Urs (2020): Educational gains of in-person vs. distance learning in primary and secondary schools. A natural experiment during the COVID-19 pandemic school closures in Switzerland. In: International Journal of Psychology. Online verfügbar unter https://onlinelibrary.wiley.com/doi/10.1002/ijop.12728.

UNICEF (2020): Eine verlorene COVID-19 Generation verhindern. Online verfügbar unter https://www.unicef.de/ informieren/aktuelles/presse/2020/covid-19-folgen-fuer-kinder/230526.

Universitätsklinikum Hamburg-Eppendorf (10.02.2021): COPSY-Studie: Kinder und Jugendliche leiden psychisch weiterhin stark unter Corona-Pandemie. Online verfügbar unter https://www.uke.de/kliniken-institute/kliniken/ kinder-und-jugendpsychiatrie-psychotherapie-und-psychosomatik/forschung/arbeitsgruppen/child-public-health/ forschung/copsy-studie.html.

Walper, Sabine; Sawatzki, Barbara; Alt, Philipp; Reim, Julia; Schmiedeberg, Claudia; Thönnissen, Carolin; Wetzel, Martin (2020): The pairfam COVID-19 survey: Design and instruments. Release Version. LMU Munich: pairfam Technical Paper 15.

Wildemann, Anja; Hosenfeld, Ingmar (2020): Bundesweite Elternbefragung zu Homeschooling während der Covid 19-Pandemie. Erkenntnisse zur Umsetzung des Homeschoolings in Deutschland. Hg. v. Universität Koblenz-Landau. Landau, zuletzt geprüft am 12.02.2021.

Wolter, Ilka; Nusser, Lena; Attig, Manja; Fackler, Sina (2020): Corona-bedingte Schulschließungen - ...und nun funktioniert alles digital? Wie Eltern mit Kindern in der 8. Klasse die Zeit der Schulschließungen in Deutschland erlebt haben. Leibniz-Institut für Bildungsverläufe e.V. Bamberg (NEPS Corona \& Bildung, 1).

Wößmann, Ludger (2020): Folgekosten ausbleibenden Lernens: Was wir über die Corona-bedingten Schulschließungen aus der Forschung lernen können. In: ifo-Schnelldienst 73 (06), S. 38-44. 
Wößmann, Ludger; Freundl, Vera; Grewenig, Elisabeth; Lergetporer, Philipp; Werner, Katharina; Zierow, Larissa (2020a): Bildung in der Coronakrise: Wie haben die Schulkinder die Zeit der Schulschließungen verbracht, und welche Bildungsmaßnahmen befürworten die Deutschen? In: ifo-Schnelldienst 73 (9), S. 25-39.

Wößmann, Ludger; Freundl, Vera; Grewenig, Elisabeth; Lergetporer, Philipp; Werner, Katharina; Zierow, Larissa (2020b): Bildung in der Coronakrise: Wie haben die Schulkinder die Zeit der Schulschließungen verbracht, und welche Bildungsmaßnahmen befürworten die Deutschen? In: ifo-Schnelldienst 73 (9), S. 25-39.

Wößmann, Ludger; Freundl, Vera; Grewenig, Elisabeth; Lergetporer, Philipp; Werner, Katharina; Zierow, Larissa (2021): Bildung erneut im Lockdown: Wie verbrachten Schulkinder die Schulschließungen Anfang 2021? In: ifo-Schnelldienst 74 (5), S. 36-52.

Wuttke, Julia; Bujard, Martin; Schöningh, Insa (2020): Coronavirus und Kindeswohl. 4 Vorschläge für eine kindgerechte Familienpolitik in Zeiten der Pandemie. In: Familienpolitische Informationen (2-2020), S. 1-4.

Zierer, Klaus (2021): Effects of Pandemic-Related School Closures on Pupils' Performance and Learning in Selected Countries: A Rapid Review. In: education sciences 11. DOI: 10.3390/educsci11060252.

Zinn, Sabine; Bayer, Michael; Entringer, Theresa; Goebel, Jan; Grabka, Markus M.; Graeber, Daniel et al. (2020): Subjektive Belastung der Eltern durch Schulschließungen zu Zeiten des Corona-bedingten Lockdowns. Hg. v. DIW Berlin (SOEPpapers on Multidisciplinary Panel Data Research, 1097).

Zoch, Gundula; Bächmann, Ann-Christin; Vicari, Basha (2020a): Care-arrangements and parental well-being during the covid-19 pandemic in Germany. Hg. v. Leibniz Institute for Educational Trajectories (LIfBi). Bamberg (LIfBi Working Paper, 91), zuletzt geprüft am 11.02.2021.

Zoch, Gundula; Bächmann, Ann-Christin; Vicari, Basha (2020b): Kinderbetreuung in der Corona-Krise: Wer betreut, wenn Schulen und Kitas schließen? Hg. v. Leibniz-Institut für Bildungsverläufe e.V. (NEPS Corona \& Bildung, 3), zuletzt geprüft am 28.06.2021.

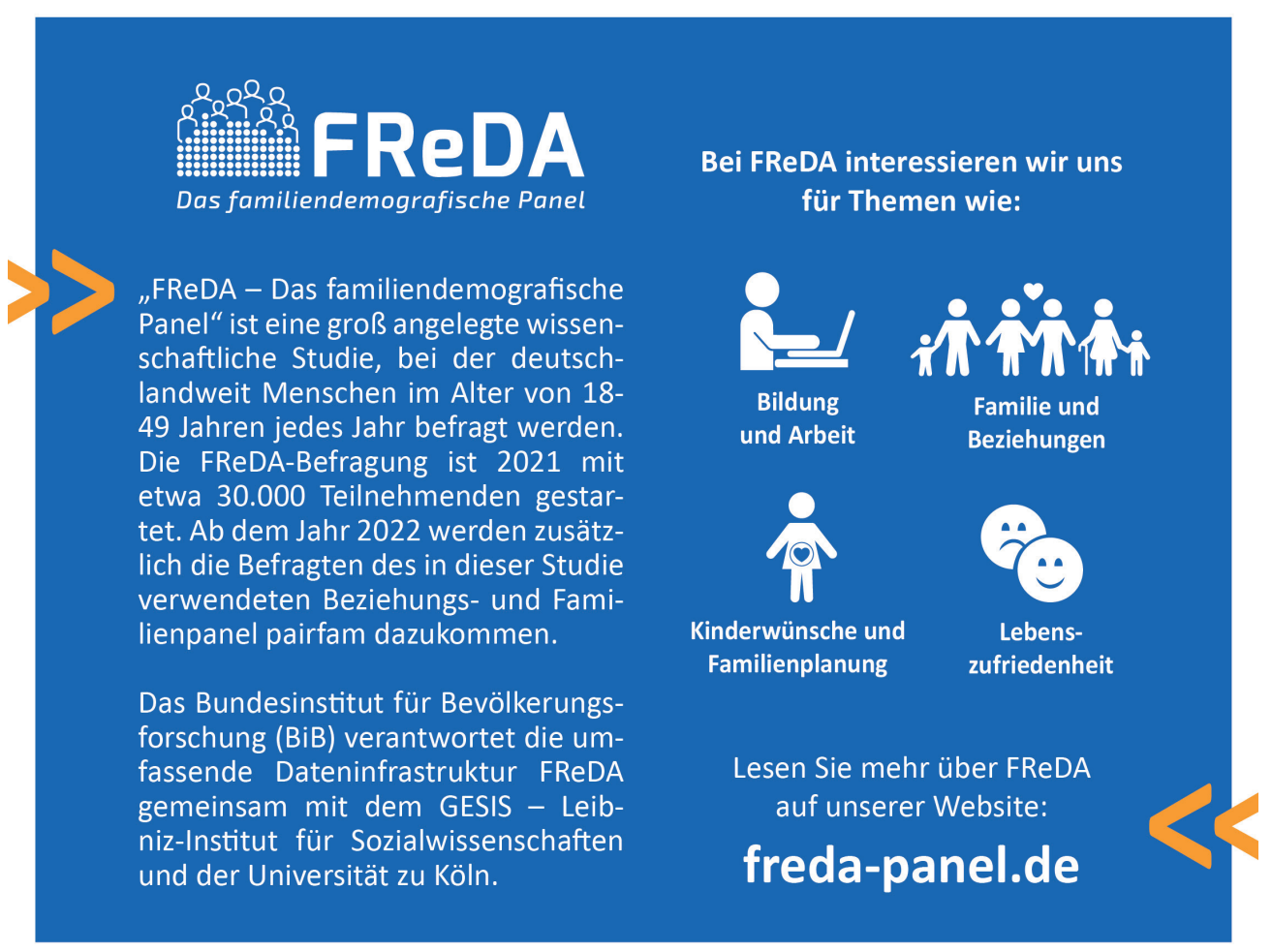


